Supporting Information

\title{
Stereoselective Allylstannane Addition for a Convergent Synthesis of a Complex Molecule
}

\author{
Alejandro Gil, Adriana Lorente, Fernando Albericio and Mercedes Álvarez \\ Institute for Research in Biomedicine, Barcelona Science Park-University of Barcelona, Baldiri Reixac 10, E- \\ 08028 Barcelona, Spain. CIBER-BBN, Networking Centre on Bioengineering, Biomaterials and Nanomedicine, \\ Department of Organic Chemistry, University of Barcelona, 08028 Barcelona, Spain; Laboratory of Organic \\ Chemistry, Faculty of Pharmacy, University of Barcelona, 08028 Barcelona, Spain.
}

$\underline{\text { mercedes.alvarez@irbbarcelona.org }}$

RECEIVED DATE (to be automatically inserted after your manuscript is accepted if required according to the journal that you are submitting your paper to) 


\section{TABLE OF CONTENTS}

SI 2

SI 3-SI 17

SI 17

SI 18-SI 19

SI 20

SI 21-SI 82
General Procedures

Experimental procedures and characterization.

NOE 1D experiment of $7 \mathbf{a}$.

Mosher's derivatization of compound $\mathbf{1 3 .}$

Bibliography

NMR data of compounds 5-S8 


\section{General Procedures.}

Tetrahydrofuran (THF) and N,N-dimethylformamide (DMF) were dried using a PureSolv solvent purification system. All other solvents and reagents were used as purchased without further purification, unless indicated. Flash column chromatography was performed on SDS silica gel (60A $35-70 \mu \mathrm{m})$ as stationary phase. Analytical TLC was done on pre-coated silica gel $60 \mathrm{~F}_{254}$ plates $(0.2$ $\mathrm{mm}$ thick, 20x20 cm) and visualized under UV light $(254$ and $360 \mathrm{~nm})$, with anisaldehyde in conc. $\mathrm{H}_{2} \mathrm{SO}_{4}$ or with phosphomolybdic acid in ethanol.. Polarimetry studies were performed on a PerkinElmer 241 or JascoP-2000 polarimeter equipped with a Na-lamp. IR spectra were recorded on a Thermo Nicolet FT-IR Nexus spectrometer. ${ }^{1} \mathrm{H}-\mathrm{NMR}$ and ${ }^{13} \mathrm{C}-\mathrm{NMR}$ were recorded on a Varian Mercury 400MHz or a Varian VNMRS500 500MHz. Chemical shifts are reported in ppm referenced to the residual solvent peaks $\left(\mathrm{CDCl}_{3}\right)$ and coupling constants are reported in $\mathrm{Hz}$. Multiplicity of the carbons was assigned with gHSQC experiments. Standard abbreviations for off-resonance decoupling were employed: $\mathrm{s}=$ singlet, $\mathrm{d}=$ doublet, $\mathrm{t}=$ triplet, $\mathrm{q}=$ quadruplet. The same abbreviations were also used for the multiplicity of signals in ${ }^{1} \mathrm{H}-\mathrm{NMR}$, as well as, bs = broad singlet, bd = broad doublet, $\mathrm{m}=$ multiplet. High Resolution Mass Spectroscopy (HRMS) was performed an Agilent LC/MSD-TOF 2006 using ESI-MS technique. 


\section{Experimental procedures and characterization}

(4S,5R)-5-(((tert-Butyldiphenylsilyl)oxy)methyl)-4-hydroxydihydrofuran-2(3H)-one (S1) ${ }^{1}$

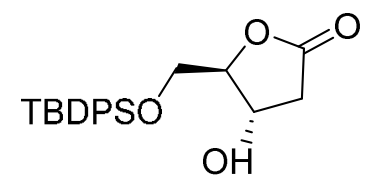

TBDPSCl (9.7 mL, $37.5 \mathrm{mmol}$.) was added to a solution of 2-D-deoxyribose (4.5 g, $34 \mathrm{mmol})$ and imidazole $(6.4 \mathrm{~g}, 95 \mathrm{mmol})$ in DMF $(150 \mathrm{~mL})$. The reaction mixture was stirred at r.t. for $16 \mathrm{~h}$. After this time DMF was evaporated, the mixture was washed with water, extracted with EtOAc, dried over $\mathrm{MgSO}_{4}$, filtered and the solvent was removed under reduced pressure. Purification by silica gel column chromatography with hexane-acetone (30:70) yielded S1 (5.1 g, 40\%) as a colorless oil. ${ }^{1} \mathrm{H}$ NMR data were in agreement with that reported in the literature. ${ }^{1}$

\section{(4S,5R)-5-(((tert-Butyldiphenylsilyl)oxy)methyl)-4-((triethylsilyl)oxy)dihydrofuran-2(3H)-one}

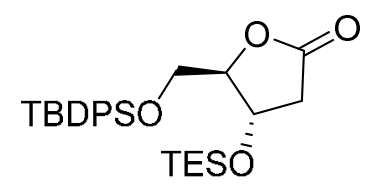

TESCl (1 mL, 5.9 mmol.) was added to a solution of alcohol S1 (2 g, $5.4 \mathrm{mmol})$ and imidazole (735 $\mathrm{mg}, 10.8 \mathrm{mmol})$ in $\mathrm{CH}_{2} \mathrm{Cl}_{2}(100 \mathrm{~mL})$. The reaction mixture was stirred at r.t. for 45 minutes. After this time, the mixture was washed with water, dried over $\mathrm{MgSO}_{4}$, filtered and the solvent was removed under reduced pressure. Purification by silica gel column chromatography with hexaneEtOAc (90:10) yielded $5(2.41 \mathrm{~g}, 92 \%)$ as a colorless oil. $[\alpha]_{\mathrm{D}}=+19.1$ (c 1.0, $\left.\mathrm{CHCl}_{3}\right)$. IR ( $\mathrm{KBr}$ film) v 2956, 2876, 1788, 1471, 1427, 1113, $702 \mathrm{~cm}^{-1} .{ }^{1} \mathrm{H}$ NMR $\left(400 \mathrm{MHz}, \mathrm{CDCl}_{3}\right) \delta 0.57$ (q, $J=7.9 \mathrm{~Hz}$, $6 \mathrm{H}), 0.93(\mathrm{t}, J=7.9 \mathrm{~Hz}, 9 \mathrm{H}), 1.05(\mathrm{~s}, 9 \mathrm{H}), 2.43$ (dd, $J=17.7,2.6 \mathrm{~Hz}, 1 \mathrm{H}), 2.89(\mathrm{dd}, J=17.7,6.7$ $\mathrm{Hz}, 1 \mathrm{H}), 3.74$ (dd, $J=11.6,2.6 \mathrm{~Hz}, 1 \mathrm{H}), 3.84$ (dd, $J=11.6,3.3 \mathrm{~Hz}, 1 \mathrm{H}), 4.35$ (dt, $J=3.3,2.6 \mathrm{~Hz}$, $1 \mathrm{H}), 4.49-4.55(\mathrm{~m}, 1 \mathrm{H}), 7.37-7.49(\mathrm{~m}, 6 \mathrm{H}), 7.62-7.66(\mathrm{~m}, 4 \mathrm{H}) .{ }^{13} \mathrm{C} \mathrm{NMR}\left(100.6 \mathrm{MHz}, \mathrm{CDCl}_{3}\right)$ $\delta 4.6$ (t), 6.6 (q), 19.1 (s) , 26.8 (q), 39.1 (t), 63.2 (t), 69.4 (d), 88.1 (d), 127.9 (d), 130.0 (d), 132.1 (s) , 132.7 (s) , 135.5 (d), 135.6 (d), 175.7 (s).HRMS (+ESI): $m / z$ calcd. for $\mathrm{C}_{27} \mathrm{H}_{40} \mathrm{O}_{4} \mathrm{KSi}_{2}(\mathrm{M}+\mathrm{K})$ 523.2097, found 523.2103. 


\section{(2RS,4S,5R)-5-(((tert-Butyldiphenylsilyl)oxy)methyl)-4-((triethylsilyl)oxy)tetrahydro furan-2-}

yl acetate (6)

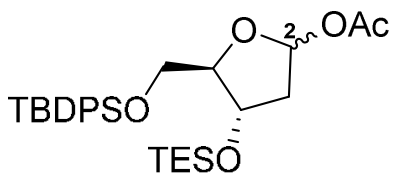

DIBAL $1 \mathrm{M}$ in toluene $(10.9 \mathrm{~mL}, 10.9 \mathrm{mmol}$.) was added to a solution of lactone $5(2.4 \mathrm{~g}, 4.95$ $\mathrm{mmol})$ in $\mathrm{CH}_{2} \mathrm{Cl}_{2}(50 \mathrm{~mL})$ at $-78^{\circ} \mathrm{C}$. The reaction mixture was stirred at this temperature for 60 minutes. After this time, a DMAP (610 mg, 4.95 mmol.) solution in $\mathrm{CH}_{2} \mathrm{Cl}_{2}(8 \mathrm{~mL}), \mathrm{Et}_{3} \mathrm{~N}(2 \mathrm{~mL}, 14$ mmol) and $\mathrm{Ac}_{2} \mathrm{O}(2.4 \mathrm{~mL}, 25 \mathrm{mmol})$ were added sequentially. The solution colour changes from colorless to orange. The mixture was warmed to $0{ }^{\circ} \mathrm{C}$ and quenched with sodium potassium tartrate $(20 \mathrm{~mL})$ and ammonium chloride $(20 \mathrm{~mL})$ saturated solutions. The mixture was extracted three times with $\mathrm{CH}_{2} \mathrm{Cl}_{2}$ and the organic layer was dried over $\mathrm{MgSO}_{4}$, filtered and the solvent was removed under reduced pressure. Purification by silica gel column chromatography with hexaneEtOAc (90:10) and 1\% of $\mathrm{Et}_{3} \mathrm{~N}$ yielded $6(2 \mathrm{~g}, 76 \%)$ as a $6(\mathrm{~A}): 4(\mathrm{~B})$ epimeric mixture in $\mathrm{C} 2$. IR $\left(\mathrm{KBr}\right.$ film) v 3071, 2956, 2876, 1750, 1471, 1427, 1234, 1112, $702 \mathrm{~cm}^{-1} .{ }^{1} \mathrm{H}$ NMR (400 MHz, $\left.\mathrm{CDCl}_{3}\right) \delta 0.53-0.64\left(\mathrm{~m}, 6 \mathrm{H}_{\mathrm{A}}+6 \mathrm{H}_{\mathrm{B}}\right), 0.90-0.99\left(\mathrm{~m}, 9 \mathrm{H}_{\mathrm{A}}+9 \mathrm{H}_{\mathrm{B}}\right), 1.05\left(\mathrm{~s}, 9 \mathrm{H}_{\mathrm{A}}\right), 1.07\left(\mathrm{~s}, 9 \mathrm{H}_{\mathrm{B}}\right), 1.89$ $\left(\mathrm{s}, 3 \mathrm{H}_{\mathrm{B}}\right), 2.01\left(\mathrm{~m}, 1 \mathrm{H}_{\mathrm{A}}\right), 2.06\left(\mathrm{~s}, 3 \mathrm{H}_{\mathrm{B}}\right), 2.17-2.23\left(\mathrm{~m}, 2 \mathrm{H}_{\mathrm{B}}\right), 2.35-2.44\left(\mathrm{~m}, 1 \mathrm{H}_{\mathrm{A}}\right), 3.67-3.78(\mathrm{~m}$, $\left.2 \mathrm{H}_{\mathrm{A}}+2 \mathrm{H}_{\mathrm{B}}\right), 3.94-3.99\left(\mathrm{~m}, 1 \mathrm{H}_{\mathrm{B}}\right), 4.19\left(\mathrm{q}, J=3.5 \mathrm{~Hz}, 1 \mathrm{H}_{\mathrm{A}}\right), 4.46\left(\mathrm{dt}, J=6.8,2.6 \mathrm{~Hz}, 1 \mathrm{H}_{\mathrm{B}}\right), 4.60(\mathrm{td}$, $\left.J=6.3,4.7 \mathrm{~Hz}, 1 \mathrm{H}_{\mathrm{A}}\right), 6.31\left(\mathrm{dd}, J=5.6,1.2 \mathrm{~Hz}, 1 \mathrm{H}_{\mathrm{A}}\right), 6.34\left(\mathrm{dd}, J=4.7,3.3 \mathrm{~Hz}, 1 \mathrm{H}_{\mathrm{B}}\right), 7.34-7.46$ $\left(\mathrm{m}, 6 \mathrm{H}_{\mathrm{A}}+6 \mathrm{H}_{\mathrm{B}}\right), 7.62-7.72\left(\mathrm{~m}, 4 \mathrm{H}_{\mathrm{A}}+4 \mathrm{H}_{\mathrm{B}}\right) .{ }^{13} \mathrm{C} \mathrm{NMR}\left(100.6 \mathrm{MHz}, \mathrm{CDCl}_{3}\right) \delta 4.7(\mathrm{t}), 4.7(\mathrm{t}), 6.7(\mathrm{q})$, $19.2(\mathrm{~s}), 19.3(\mathrm{~s}), 21.2(\mathrm{q}), 21.4(\mathrm{q}), 26.8(\mathrm{q}), 41.2(\mathrm{t}), 41.7(\mathrm{t}), 63.2(\mathrm{t}), 63.5(\mathrm{t}), 70.4(\mathrm{~d}), 71.4(\mathrm{~d})$, 87.7 (d), 88.7 (d), 98.1 (d), 99.3 (d), 127.7 (d), 127.7 (d), 127.7 (d), 129.7 (d), 129.7 (d), 129.7 (d), 133.0 (s), 133.3 (s), 135.5 (d), 135.6 (d), 135.6 (d), 135.6 (d), 170.4 (s), 170.7 (s). HRMS (+ESI): $m / z$ calcd. for $\mathrm{C}_{29} \mathrm{H}_{48} \mathrm{O}_{5} \mathrm{NSi}_{2}\left(\mathrm{M}+\mathrm{NH}_{4}\right)$ 546.3066, found 546.3066.

\section{(((2R,3S,5R)-5-Allyl-2-(((tert-butyldiphenylsilyl)oxy)methyl)-3-(triethylsilyl)oxy)}

tetrahydrofuran (7a)

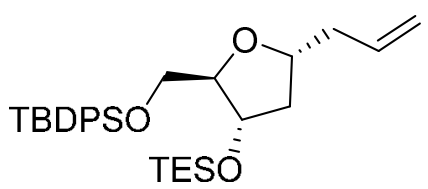

Allyltrimethylsilane $(2.1 \mathrm{~mL}, 13.2 \mathrm{mmol})$ and $\mathrm{BF}_{3} \cdot \mathrm{Et}_{2} \mathrm{O}(0.9 \mathrm{~mL}, 7.3 \mathrm{mmol})$ were added sequentially and slowly to a solution of acetal acetate $6(3.5 \mathrm{~g}, 6.65 \mathrm{mmol})$ in $\mathrm{CH}_{2} \mathrm{Cl}_{2}(80 \mathrm{~mL})$ at $-78{ }^{\circ} \mathrm{C}$. The reaction mixture was stirred at this temperature for 5 minutes and then at room 
temperature for 30 minutes. After this time, the reaction was quenched at $-78{ }^{\circ} \mathrm{C}$ with the addition of a mixture of $\mathrm{CH}_{2} \mathrm{Cl}_{2}(15 \mathrm{~mL}): \mathrm{MeOH}(15 \mathrm{~mL}): \mathrm{Et}_{3} \mathrm{~N}(15 \mathrm{~mL})$. The mixture was warmed to room temperature and extracted with $\mathrm{CH}_{2} \mathrm{Cl}_{2}$. The reaction crude was dried over $\mathrm{Na}_{2} \mathrm{SO}_{4}$. The crude was dissolved in $25 \mathrm{~mL}$ of $\mathrm{CH}_{2} \mathrm{Cl}_{2}$ and TESCl $(0.25 \mathrm{~mL}, 1.5 \mathrm{mmol})$, imidazole (204 mg, $\left.3 \mathrm{mmol}\right)$ and DMAP (12 mg, $0.1 \mathrm{mmol}$ ) were added to reprotect $7 \mathbf{b}$. The reaction was stirred for 15 minutes. After this time, the mixture was washed with water, dried over $\mathrm{MgSO}_{4}$, filtered and the solvent was removed under reduced pressure. Purification by silica gel column chromatography with hexaneEtOAc $(95: 5)$ yielded $7 \mathbf{a}(2.74 \mathrm{~g}, 81 \%)$ as a single diasteromer colorless oil. $[\alpha]_{\mathrm{D}}=+19.6$ (c 1.0, $\left.\mathrm{CHCl}_{3}\right)$. IR (KBr film) v 3071, 2956, 2876, 1471, 1427, 1112, $701 \mathrm{~cm}^{-1} .{ }^{1} \mathrm{H}$ NMR (400 MHz, $\left.\mathrm{CDCl}_{3}\right) \delta 0.59$ (q, $\left.J=7.9 \mathrm{~Hz}, 6 \mathrm{H}\right), 0.94(\mathrm{t}, J=7.9 \mathrm{~Hz}, 9 \mathrm{H}), 1.06(\mathrm{~s}, 9 \mathrm{H}), 1.68(\mathrm{ddd}, J=12.6,7.0$, $5.3 \mathrm{~Hz}, 1 \mathrm{H}), 2.20(\mathrm{dt}, J=12.6,6.5 \mathrm{~Hz}, 1 \mathrm{H}), 2.28-2.38(\mathrm{~m}, 1 \mathrm{H}), 2.47$ (ddt, $J=13.6,6.5,1.4 \mathrm{~Hz}$, $1 \mathrm{H}), 3.66(\mathrm{~d}, J=4.1 \mathrm{~Hz}, 2 \mathrm{H}), 3.92(\mathrm{q}, J=4.1 \mathrm{~Hz}, 1 \mathrm{H}), 4.09-4.18(\mathrm{~m}, 1 \mathrm{H}), 4.50(\mathrm{ddd}, J=6.5,5.3$, $3.9 \mathrm{~Hz}, 1 \mathrm{H}), 5.01-5.14(\mathrm{~m}, 2 \mathrm{H}), 5.76-5.90(\mathrm{~m}, 1 \mathrm{H}), 7.34-7.46(\mathrm{~m}, 6 \mathrm{H}), 7.66-7.73(\mathrm{~m}, 4 \mathrm{H}) .{ }^{13} \mathrm{C}$ NMR (100.6 MHz, $\left.\mathrm{CDCl}_{3}\right) \delta 4.7(\mathrm{q}), 6.8(\mathrm{t}), 19.2(\mathrm{~s}), 26.8(\mathrm{q}), 40.5(\mathrm{t}), 40.8(\mathrm{t}), 64.2(\mathrm{t}), 73.0(\mathrm{~d})$, 78.2 (d), 86.2 (d), 116.6 (t), 127.6 (d), 127.6 (d), 129.6 (d), 129.6 (d), 133.4 (s), 133.5 (s), 135.3 (d), 135.6 (d), 135.7 (d). HRMS (+ESI): $\mathrm{m} / z$ calcd. for $\mathrm{C}_{30} \mathrm{H}_{50} \mathrm{O}_{3} \mathrm{NSi}_{2}(\mathrm{M}+\mathrm{NH} 4)$ 528.3324, found 528.3322 .

\section{2-((2S,4S,5R)-5-(((tert-Butyldiphenylsilyl)oxy)methyl)-4-((triethylsilyl)oxy)tetrahydrofuran-2-}

\section{yl)acetaldehyde (8)}

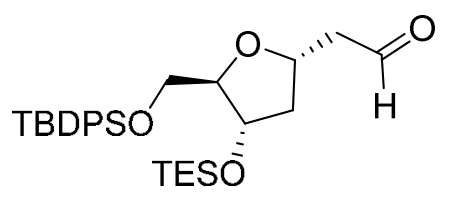

Ozone was bubbled through a solution of $7 \mathbf{a}(2.74 \mathrm{~g}, 5.37 \mathrm{mmol})$ in of $\mathrm{CH}_{2} \mathrm{Cl}_{2}(80 \mathrm{~mL})$ and of $\mathrm{MeOH}(20 \mathrm{~mL})$ at $-78{ }^{\circ} \mathrm{C}$ until the solution became intense blue. Then $\mathrm{PPh}_{3}(1.83 \mathrm{~g}, 7 \mathrm{mmol})$ was added and the reaction was stirred for $2 \mathrm{~h}$ at room temperature. The mixture was evaporated and purified by silica gel column chromatography with hexane-EtOAc (95:5) to yield $\mathbf{8}(2.64 \mathrm{~g}, 96 \%)$ as a colorless oil. $[\alpha]_{\mathrm{D}}=+19.6\left(\mathrm{c} 1.0, \mathrm{CHCl}_{3}\right)$. IR ( $\mathrm{KBr}$ film) $\vee 3071,2956,2876,1471,1427,1112$, $701 \mathrm{~cm}^{-1} .{ }^{1} \mathrm{H}$ NMR $\left(400 \mathrm{MHz}, \mathrm{CDCl}_{3}\right) \delta 0.59$ (q, $\left.J=7.9 \mathrm{~Hz}, 6 \mathrm{H}\right), 0.94(\mathrm{t}, J=7.9 \mathrm{~Hz}, 9 \mathrm{H}), 1.06(\mathrm{~s}$, 9H), 1.69 (ddd, $J=12.8,5.4,4.0 \mathrm{~Hz}, 1 \mathrm{H}), 2.35$ (ddd, $J=12.8,7.6,6.1 \mathrm{~Hz}, 1 \mathrm{H}), 2.66$ (ddd, $J=16.6$, 5.4, $2.0 \mathrm{~Hz}, 1 \mathrm{H}), 2.89$ (ddd, $J=16.6,7.6,2.0 \mathrm{~Hz}, 1 \mathrm{H}), 3.57-3.69(\mathrm{~m}, 2 \mathrm{H}), 3.97(\mathrm{dt}, J=4.8,3.4$ $\mathrm{Hz}, 1 \mathrm{H}), 4.50$ (ddd, $J=6.1,4.0,3.4 \mathrm{~Hz}, 1 \mathrm{H}), 4.58$ (tt, $J=7.6,5.4 \mathrm{~Hz}, 1 \mathrm{H}), 7.35-7.46$ (m, 6H), $7.63-7.71(\mathrm{~m}, 4 \mathrm{H}), 9.81(\mathrm{t}, J=2.0 \mathrm{~Hz}, 1 \mathrm{H}) .{ }^{13} \mathrm{C} \mathrm{NMR}\left(100.6 \mathrm{MHz}, \mathrm{CDCl}_{3}\right) \delta 4.7$ (t), 6.7 (q), 19.2 
(s), $26.8(\mathrm{q}), 40.8(\mathrm{t}), 50.5(\mathrm{t}), 64.2(\mathrm{t}), 73.2$ (d), 73.9 (d), 86.9 (d), 127.7 (d), 127.7 (d), 129.7 (d), 129.7 (d), 133.2 (s), 133.3 (s), 135.6 (d), 135.6 (d), 201.6 (d). HRMS (+ESI): $\mathrm{m} / z$ calcd. for $\mathrm{C}_{29} \mathrm{H}_{44} \mathrm{NaO}_{4} \mathrm{Si}_{2}(\mathrm{M}+\mathrm{Na})$ 535.267, found 535.2676.

\section{(R)-3-((E)-4-((2R,4S,5R)-5-(((tert-Butyldiphenylsilyl)oxy)methyl)-4-}

((triethylsilyl)oxy)tetrahydrofuran-2-yl)but-2-enoyl)-4-phenyloxazolidin-2-one (S2).

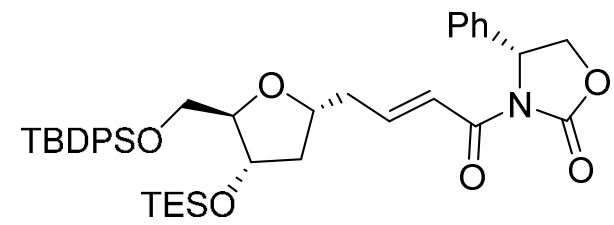

A $1 \mathrm{M}$ solution of NaHMDS in THF $(7.1 \mathrm{~mL}, 7.1 \mathrm{mmol})$ was added to a solution of phosphonate $9^{2}(3.6 \mathrm{~g}, 10.6 \mathrm{mmol})$ in THF $(70 \mathrm{~mL})$. After $10 \mathrm{~min}$, a solution of aldehyde $8(2.96 \mathrm{~g}$, $5.7 \mathrm{mmol})$ in THF $(15 \mathrm{~mL})$ was added dropwise, and the mixture was stirred at r.t. for 90 minutes. After this time, $\mathrm{KH}_{2} \mathrm{PO}_{4} \cdot \mathrm{NaOH} \mathrm{pH}=7$ buffer was added and the solvent was removed under reduced pressure. The residue was disolved in water and extracted with EtOAc. The organic layer was dried over $\mathrm{MgSO}_{4}$, filtered and the solvent was removed under reduced pressure. Purification by silica gel column chromatography with hexane-EtOAc (90:10 to 80:20) yielded S2 (3.26 g, 82\%) as a colorless foam. $[\alpha]_{\mathrm{D}}=-19.0\left(\mathrm{c} 1.0, \mathrm{CHCl}_{3}\right)$. IR $(\mathrm{KBr}$ film $) \vee 2955,2866,2857,1781,1689$, 1629, 1367, 1347, 1196, 1112, $702 \mathrm{~cm}^{-1} .{ }^{1} \mathrm{H}$ NMR (400 MHz, $\left.\mathrm{CDCl}_{3}\right) \delta 0.57$ (q, $\left.J=7.9 \mathrm{~Hz}, 6 \mathrm{H}\right)$, $0.93(\mathrm{t}, J=7.9 \mathrm{~Hz}, 9 \mathrm{H}), 1.04(\mathrm{~s}, 9 \mathrm{H}), 1.67(\mathrm{ddd}, J=13.0,6.4,4.8 \mathrm{~Hz}, 1 \mathrm{H}), 2.22(\mathrm{dt}, J=13.0,6.6$ $\mathrm{Hz}, 1 \mathrm{H}), 2.49-2.59(\mathrm{~m}, 1 \mathrm{H}), 2.63-2.74(\mathrm{~m}, 1 \mathrm{H}), 3.62(\mathrm{dd}, J=3.9,1.1 \mathrm{~Hz}, 2 \mathrm{H}), 3.91$ (q, $J=3.9$ $\mathrm{Hz}, 1 \mathrm{H}), 4.19(\mathrm{~m}, 1 \mathrm{H}), 4.28(\mathrm{~m}, 1 \mathrm{H}), 4.47$ (ddd, $J=6.4,4.8,3.6 \mathrm{~Hz}, 1 \mathrm{H}), 4.69(\mathrm{t}, J=8.7 \mathrm{~Hz}, 1 \mathrm{H})$, $5.49(\mathrm{dd}, J=8.7,3.9 \mathrm{~Hz}, 1 \mathrm{H}), 7.09$ (dt, $J=15.4,7.3 \mathrm{~Hz}, 1 \mathrm{H}), 7.29-7.44(\mathrm{~m}, 12 \mathrm{H}), 7.62-7.69(\mathrm{~m}$, 4H). ${ }^{13} \mathrm{C}$ NMR (100.6 MHz, $\left.\mathrm{CDCl}_{3}\right) \delta 4.7(\mathrm{t}), 6.8(\mathrm{q}), 19.2(\mathrm{~s}), 26.8(\mathrm{q}), 39.7(\mathrm{t}), 40.5(\mathrm{t}), 57.7(\mathrm{~d})$, $64.1(\mathrm{t}), 69.9$ (t), 73.0 (d), 77.5 (d), 86.5 (d), 121.9 (d), 126.0 (d), 127.6 (d), 128.6 (d), 129.1 (d), 129.6 (d), 129.6 (d), 133.2 (s), 133.4 (s), 135.6 (d), 135.6 (d), 139.1 (s), 148.3 (d), 153.6 (s), 164.3 (s). HRMS (+ESI): $m / z$ calcd. for $\mathrm{C}_{40} \mathrm{H}_{53} \mathrm{NNaO}_{6} \mathrm{Si}_{2}(\mathrm{M}+\mathrm{Na}) 722.3304$, found 722.3299 . 


\section{(R)-3-((S)-4-((2R,4S,5R)-5-(((tert-Butyldiphenylsilyl)oxy)methyl)-4-((triethylsilyl)oxy)}

tetrahydrofuran-2-yl)-3-methylbutanoyl)-4-phenyloxazolidin-2-one (10)

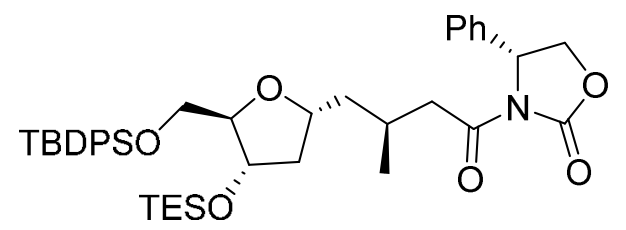

A $3 \mathrm{M}$ solution of $\mathrm{MeMgBr}$ in THF $(1.36 \mathrm{~mL}, 4.1 \mathrm{mmol})$ was added to a solution of $\mathrm{CuBr} \cdot \mathrm{Me}_{2} \mathrm{~S}$ (760 mg, $3.7 \mathrm{mmol})$ in THF $(75 \mathrm{~mL})$ at $-40{ }^{\circ} \mathrm{C}$, and the mixture was stirred at $-40{ }^{\circ} \mathrm{C}$ for $1 \mathrm{~h}$. The solution was cooled to $-78{ }^{\circ} \mathrm{C}$ and $\mathrm{BF}_{3} \cdot \mathrm{Et}_{2} \mathrm{O}(0.46 \mathrm{~mL}, 3.7 \mathrm{mmol})$ and a solution of oxazolidinone S2 $(2.81 \mathrm{~g}, 3.4 \mathrm{mmol})$ in THF were added. The reaction mixture was stirred at $-78{ }^{\circ} \mathrm{C}$ for $1 \mathrm{~h}$, slowly warmed to r.t. during $2 \mathrm{~h}$ and stirred at r.t. for 1 hour more. After this time, sat. $\mathrm{NH}_{4} \mathrm{Cl}$ was added and the solvent was removed under reduced pressure. The residue was diluted in sat. $\mathrm{NH}_{4} \mathrm{Cl}$ and extracted with $\mathrm{Et}_{2} \mathrm{O}$. The organic solution was dried over $\mathrm{MgSO}_{4}$, filtered and the solvent was removed under reduced pressure. Purification by silica gel column chromatography with hexaneEtOAc (90:10 to 80:20) yielded $\mathbf{1 0}(2.41 \mathrm{~g}, 91 \%)$ as a colorless oil. $[\alpha]_{\mathrm{D}}=-11.6\left(\mathrm{c} 1.0, \mathrm{CHCl}_{3}\right)$. IR $\left(\mathrm{KBr}\right.$ film) $\vee 2956,2876,1784,1707,1457,1383,1322,1196,1112 \mathrm{~cm}^{-1} .{ }^{1} \mathrm{H}$ NMR (400 MHz, $\left.\mathrm{CDCl}_{3}\right) \delta 0.58(\mathrm{q}, J=7.9 \mathrm{~Hz}, 6 \mathrm{H}), 0.93(\mathrm{~d}, J=6.7 \mathrm{~Hz}, 3 \mathrm{H}), 0.93(\mathrm{t}, J=7.9 \mathrm{~Hz}, 9 \mathrm{H}), 1.05(\mathrm{~s}, 9 \mathrm{H})$, $1.47-1.54(\mathrm{~m}, 1 \mathrm{H}), 1.56-1.61(\mathrm{~m}, 1 \mathrm{H}), 1.61-1.66(\mathrm{~m}, 1 \mathrm{H}), 2.10-2.17(\mathrm{~m}, 1 \mathrm{H}), 2.20(\mathrm{dd}, J=$ 12.4, $6.3 \mathrm{~Hz}, 1 \mathrm{H}), 2.84(\mathrm{dd}, J=16.7,6.7 \mathrm{~Hz}, 1 \mathrm{H}), 2.95(\mathrm{dd}, J=16.7,6.7 \mathrm{~Hz}, 1 \mathrm{H}), 3.64(\mathrm{dd}, J=$ 11.0, $3.8 \mathrm{~Hz}, 1 \mathrm{H}), 3.70(\mathrm{dd}, J=11.0,3.8 \mathrm{~Hz}, 1 \mathrm{H}), 3.81(\mathrm{dt}, J=4.8,3.7 \mathrm{~Hz}, 1 \mathrm{H}), 4.07-4.13$ (m, $1 \mathrm{H}), 4.24(\mathrm{dd}, J=8.8,3.6 \mathrm{~Hz}, 1 \mathrm{H}), 4.48(\mathrm{td}, J=6.5,4.6 \mathrm{~Hz}, 1 \mathrm{H}), 4.62(\mathrm{t}, J=8.8 \mathrm{~Hz}, 1 \mathrm{H}), 5.39$ (dd, $J=8.8,3.6 \mathrm{~Hz}, 1 \mathrm{H}), 7.26-7.46(\mathrm{~m}, 11 \mathrm{H}), 7.64-7.75(\mathrm{~m}, 4 \mathrm{H}) .{ }^{13} \mathrm{C} \mathrm{NMR}\left(100.6 \mathrm{MHz}, \mathrm{CDCl}_{3}\right) \delta$ $4.7(\mathrm{t}), 6.8(\mathrm{q}), 19.2(\mathrm{~s}), 20.1(\mathrm{q}), 26.8(\mathrm{q}), 27.5(\mathrm{~d}), 41.6(\mathrm{t}), 42.3(\mathrm{t}), 42.9(\mathrm{t}), 57.6(\mathrm{~d}), 64.1(\mathrm{t}), 69.8$ (t), 72.6 (d), 76.8 (d), 85.5 (d), 125.9 (d), 127.6 (d), 128.6 (d), 129.1 (d), 129.5 (d), 129.6 (d), 133.4 (s), 133.5 (s), 135.6 (d), 135.7 (d), 139.2 (s), 153.7 (s), 171.9 (s). HRMS (+ESI): m/z calcd. for $\mathrm{C}_{41} \mathrm{H}_{57} \mathrm{O}_{6} \mathrm{NNaSi}_{2}(\mathrm{M}+\mathrm{Na})$ 738.3617, found 738.3617 .

\section{(R)-4-((2R,4S,5R)-5-(((tert-Butyldiphenylsilyl)oxy)methyl)-4-((triethylsilyl)oxy)}

tetrahydrofuran-2-yl)-3-methylbutan-1-ol (S3)

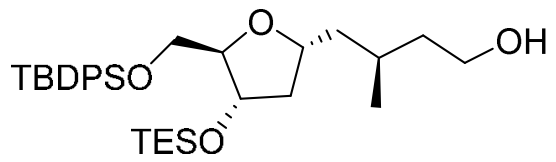


A $2 \mathrm{M}$ solution of $\mathrm{LiBH}_{4}$ in THF $(3.1 \mathrm{~mL}, 6.21 \mathrm{mmol})$ was added to a solution of oxazolidinone $\mathbf{1 0}$ $(1.85 \mathrm{~g}, 2.58 \mathrm{mmol})$ in $\mathrm{Et}_{2} \mathrm{O}(65 \mathrm{~mL}): \mathrm{MeOH}(3 \mathrm{~mL})$ at $-10^{\circ} \mathrm{C}$ and the reaction mixture was stirred at $0{ }^{\circ} \mathrm{C}$ for $1 \mathrm{~h}$. After this time, a $1 \mathrm{M}$ solution of $\mathrm{NaOH}$ was added and the mixture was extracted with EtOAc, dried over $\mathrm{MgSO}_{4}$, filtered and the solvent was removed under reduced pressure. Purification by silica gel column chromatography with hexane-EtOAc (90:10 to 85:15) yielded $\mathbf{S 3}$ $(0.85 \mathrm{~g}, 65 \%)$ as a colorless oil. $[\alpha]_{\mathrm{D}}=+27.1\left(\mathrm{c} 1.0, \mathrm{CHCl}_{3}\right)$. IR ( $\mathrm{KBr}$ film) $\vee 3404$ (br), 2955, 2931, 2875, 1463, 1428, 1239, $1113 \mathrm{~cm}^{-1} .{ }^{1} \mathrm{H} \mathrm{NMR}\left(400 \mathrm{MHz}, \mathrm{CDCl}_{3}\right) \delta 0.58(\mathrm{q}, J=7.9 \mathrm{~Hz}, 6 \mathrm{H}), 0.93(\mathrm{t}$, $J=7.8 \mathrm{~Hz}, 9 \mathrm{H}), 0.94(\mathrm{~d}, J=6.8 \mathrm{~Hz}, 3 \mathrm{H}), 1.06(\mathrm{~s}, 9 \mathrm{H}), 1.38-1.64(\mathrm{~m}, 5 \mathrm{H}), 1.74(\mathrm{dt}, J=12.8,6.6$ $\mathrm{Hz}, 1 \mathrm{H}), 2.24(\mathrm{dt}, J=12.8,6.5 \mathrm{~Hz}, 1 \mathrm{H}), 3.61-3.75(\mathrm{~m}, 4 \mathrm{H}), 3.86(\mathrm{q}, J=3.9 \mathrm{~Hz}, 1 \mathrm{H}), 4.15-4.24$ $(\mathrm{m}, 1 \mathrm{H}), 4.46-4.53(\mathrm{~m}, 1 \mathrm{H}), 7.35-7.45(\mathrm{~m}, 6 \mathrm{H}), 7.66-7.73(\mathrm{~m}, 4 \mathrm{H}) .{ }^{13} \mathrm{C} \mathrm{NMR}(100.6 \mathrm{MHz}$, $\left.\mathrm{CDCl}_{3}\right) \delta 4.7(\mathrm{t}), 6.8(\mathrm{q}), 19.2(\mathrm{~s}), 20.4(\mathrm{q}), 26.8(\mathrm{q}), 27.2(\mathrm{~d}), 39.7(\mathrm{t}), 41.6(\mathrm{t}), 43.3(\mathrm{t}), 61.1(\mathrm{t}), 64.2$ (t), 72.7 (d), 76.8 (d), 85.7 (d), 127.6 (d), 127.6 (d), 129.6 (d), 129.6 (d), 133.4 (s), 133.5 (s), 135.6 (d), 135.7 (d). HRMS (+ESI): $m / z$ calcd. for $\mathrm{C}_{32} \mathrm{H}_{52} \mathrm{O}_{4} \mathrm{NaSi}_{2}(\mathrm{M}+\mathrm{Na})$ 579.3296, found 579.3269.

\section{(S)-4-((2R,4S,5R)-5-(((tert-Butyldiphenylsilyl)oxy)methyl)-4-((triethylsilyl)oxy)}

tetrahydrofuran-2-yl)-3-methylbutanal (2)

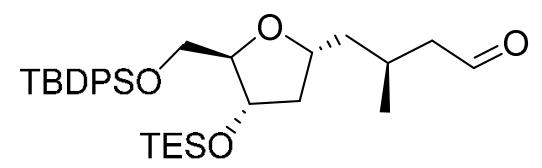

Dess-Martin periodinane $(823 \mathrm{mg}, 1.94 \mathrm{mmol})$ was added to a solution of alcohol $\mathbf{S 3}(830 \mathrm{mg}, 1.5$ mmol) in $\mathrm{CH}_{2} \mathrm{Cl}_{2}(30 \mathrm{~mL})$ and the mixture was stirred for 30 minutes. The reaction mixture was diluted with sat. $\mathrm{Na}_{2} \mathrm{~S}_{2} \mathrm{O}_{3}$ and sat. $\mathrm{NaHCO}_{3}$ and the residue was extracted with $\mathrm{CH}_{2} \mathrm{Cl}_{2}$. The organic extracts were dried over $\mathrm{MgSO}_{4}$, filtered and concentrated under reduced pressure. Purification by silica gel column chromatography with hexane-EtOAc (9:1) yielded 2 (791 $\mathrm{mg}, 95 \%)$ as a colorless oil. $[\alpha]_{\mathrm{D}}=+25.3\left(\mathrm{c} 1.0, \mathrm{CHCl}_{3}\right)$. IR $(\mathrm{KBr}$ film) $\vee 2956,2930,2857,1727,1472,1428,1252,1112$ $\mathrm{cm}^{-1} .{ }^{1} \mathrm{H}$ NMR $\left(400 \mathrm{MHz}, \mathrm{CDCl}_{3}\right) \delta 0.59(\mathrm{t}, J=7.9 \mathrm{~Hz}, 6 \mathrm{H}), 0.94(\mathrm{t}, J=7.9 \mathrm{~Hz}, 9 \mathrm{H}), 1.01(\mathrm{~d}, J=$ $6.5 \mathrm{~Hz}, 3 \mathrm{H}), 1.06$ (s, 9H), 1.50 (ddd, $J=13.7,6.7,5.0 \mathrm{~Hz}, 1 \mathrm{H}), 1.62$ (ddd, $J=12.2,7.6,5.9 \mathrm{~Hz}$, 1H), $1.72(\mathrm{ddd}, J=13.7,8.3,6.4 \mathrm{~Hz}, 1 \mathrm{H}), 2.16-2.30(\mathrm{~m}, 3 \mathrm{H}), 2.46-2.53(\mathrm{~m}, 1 \mathrm{H}), 3.66$ (dd, $J=$ 11.0, $3.9 \mathrm{~Hz}, 1 \mathrm{H}), 3.71(\mathrm{dd}, J=11.0,3.9 \mathrm{~Hz}, 1 \mathrm{H}), 3.85$ (q, $J=3.9 \mathrm{~Hz}, 1 \mathrm{H}), 4.15(\mathrm{tdd}, J=8.0,6.4$, $5.0 \mathrm{~Hz}, 1 \mathrm{H}), 4.46-4.52(\mathrm{~m}, 1 \mathrm{H}), 7.33-7.46(\mathrm{~m}, 6 \mathrm{H}), 7.65-7.72(\mathrm{~m}, 4 \mathrm{H}), 9.72-9.74(\mathrm{~m}, 1 \mathrm{H}) .{ }^{13} \mathrm{C}$ NMR (100.6 MHz, $\left.\mathrm{CDCl}_{3}\right) \delta 4.7$ (t), $6.8(\mathrm{q}), 19.2(\mathrm{~s}), 20.5$ (q), $25.8(\mathrm{~d}), 26.8(\mathrm{q}), 41.6(\mathrm{t}), 43.2(\mathrm{t})$, $50.6(\mathrm{t}), 64.1(\mathrm{t}), 72.7$ (d), 76.3 (d), 85.8 (d), 127.6 (d), 127.6 (d), 129.6 (d), 129.6 (d), $133.4(\mathrm{~s})$, 
133.5 (s), 135.6 (d), 135.6 (d), 202.9 (d). HRMS (+ESI): $m / z$ calcd. for $\mathrm{C}_{32} \mathrm{H}_{51} \mathrm{O}_{4} \mathrm{Si}_{2}(\mathrm{M}+\mathrm{H})$ 555.3320 , found 555.3326 .

6-(tert-Butyl) 1-methyl (R,E)-2-methyl-4-((triisopropylsilyl)oxy)hex-2-enedioate (S4).<smiles>CCOC(=O)C[C@@H]1C=C(C(=O)OC)[TeH][GeH2]1</smiles>

A $2 \mathrm{M}$ solution of LDA in THF $(2.7 \mathrm{~mL}, 5.4 \mathrm{mmol})$ was slowly added to a solution of (1mehtoxycarbonylethyl)triphenylphosphonium bromide ${ }^{3}(2.3 \mathrm{~g}, 5.4 \mathrm{mmol})$ in $\mathrm{CH}_{2} \mathrm{Cl}_{2}(140 \mathrm{~mL})$ at $78{ }^{\circ} \mathrm{C}$. After $5 \mathrm{~min}$, the reaction was warmed to $0^{\circ} \mathrm{C}$ and a solution of aldehyde $4^{2}(1.5 \mathrm{~g}, 4.5 \mathrm{mmol})$ in THF $(10 \mathrm{~mL})$ was added dropwise, and the mixture was stirred $1 \mathrm{~h}$ at $0{ }^{\circ} \mathrm{C}$ and $1 \mathrm{~h}$ at r.t. The reaction was quenched by the addition of $100 \mathrm{~mL}$ of $\mathrm{NH}_{4} \mathrm{Cl}$ saturated solution. The mixture was extracted three times with $\mathrm{CH}_{2} \mathrm{Cl}_{2}(75 \mathrm{~mL})$. The organic layer was dried over $\mathrm{MgSO}_{4}$, filtered and the solvent was removed under reduced pressure. Purification by silica gel column chromatography with hexane-EtOAc (95:5) yielded $\mathbf{S} 4(1.6 \mathrm{~g}, 87 \%)$ as a colorless oil. $[\alpha]_{\mathrm{D}}=+9.6\left(\mathrm{c} 1.0, \mathrm{CHCl}_{3}\right)$. IR $(\mathrm{KBr}$ film $) \vee 2944,2867,1723,1463,1248,1159,887 \mathrm{~cm}^{-1} .{ }^{1} \mathrm{H}$ NMR $\left(400 \mathrm{MHz}, \mathrm{CDCl}_{3}\right) \delta 1.01-$ $1.06(\mathrm{~m}, 21 \mathrm{H}), 1.42(\mathrm{~s}, 9 \mathrm{H}), 1.86(\mathrm{~d}, J=1.4 \mathrm{~Hz}, 3 \mathrm{H}), 2.39(\mathrm{dd}, J=14.6,6.3 \mathrm{~Hz}, 1 \mathrm{H}), 2.57(\mathrm{dd}, J=$ 14.6, $6.3 \mathrm{~Hz}, 1 \mathrm{H}), 3.75(\mathrm{~s}, 3 \mathrm{H}), 5.00(\mathrm{dt}, J=8.7,6.3 \mathrm{~Hz}, 1 \mathrm{H}), 6.72(\mathrm{dq}, J=8.7,1.4 \mathrm{~Hz}, 1 \mathrm{H}) .{ }^{13} \mathrm{C}$ NMR (100.6 MHz, $\left.\mathrm{CDCl}_{3}\right) \delta 12.3$ (d), 13.0 (q), 17.9 (q), 28.0 (q), 44.4 (t), 51.9 (q), 66.8 (d), 80.7 (s), 126.4 (s), 143.7 (d), 168.3 (s), 169.6 (s). HRMS (+ESI): $m / z$ calcd. for $\mathrm{C}_{21} \mathrm{H}_{40} \mathrm{O}_{5} \mathrm{NaSi}(\mathrm{M}+\mathrm{Na}$ ) 423.2537, found 423.2537 .

tert-Butyl (R,E)-6-hydroxy-5-methyl-3-((triisopropylsilyl)oxy)hex-4-enoate (11).<smiles>C/C(=C\[C@@H]([TeH2])CC(=O)OCC(C)C)CO</smiles>

A $1 \mathrm{M}$ solution of DIBAL in THF $(31.6 \mathrm{~mL}, 31.6 \mathrm{mmol})$ was slowly added to a solution of S4 $(5.76 \mathrm{~g}, 14.37 \mathrm{mmol})$ in THF $(210 \mathrm{~mL})$ at $-78{ }^{\circ} \mathrm{C}$ and the reaction mixture was stirred for 15 minutes. After this time the reaction was warmed to r.t and stirred $1 \mathrm{~h}$. The reaction was quenched by the addition of EtOAc $(10 \mathrm{~mL})$ and Rochelle's Salt saturated solution $(150 \mathrm{~mL})$. The mixture was stirred $2 \mathrm{~h}$ and filtered through celite. The pad was washed several times with EtOAc. The organic solvents were evaporated and the crude was extracted three times with $\mathrm{CH}_{2} \mathrm{Cl}_{2}$. This organic layer was dried over $\mathrm{MgSO}_{4}$, filtered and the solvent was removed under reduced pressure. 
Purification by silica gel column chromatography with hexane-EtOAc (9:1) yielded 11 (3.89 g, $75 \%)$ as a colorless oil. $[\alpha]_{\mathrm{D}}=+8.7\left(\mathrm{c} 1.0, \mathrm{CHCl}_{3}\right)$. IR (KBr film) $\vee 3444$ (br), 2943, 2866, 1731, 1464, 1368, 1147, 1084, $883 \mathrm{~cm}^{-1} .{ }^{1} \mathrm{H}$ NMR (400 MHz, $\left.\mathrm{CDCl}_{3}\right) \delta 1.00-1.06(\mathrm{~m}, 21 \mathrm{H}), 1.41(\mathrm{~s}$, 9H), $1.68(\mathrm{~d}, J=1.2 \mathrm{~Hz}, 3 \mathrm{H}), 2.34(\mathrm{dd}, J=14.1,6.9 \mathrm{~Hz}, 1 \mathrm{H}), 2.54(\mathrm{dd}, J=14.1,6.1 \mathrm{~Hz}, 1 \mathrm{H}), 3.98$ $(\mathrm{s}, 2 \mathrm{H}), 4.89-4.97(\mathrm{~m}, 1 \mathrm{H}), 5.47(\mathrm{dq}, J=8.8,1.2 \mathrm{~Hz}, 1 \mathrm{H}) .{ }^{13} \mathrm{C} \mathrm{NMR}\left(100.6 \mathrm{MHz}, \mathrm{CDCl}_{3}\right) \delta 12.3$ (d), 14.2 (q), 17.9 (q), 28.0 (q), 45.4 (t), 66.5 (d), 68.1 (t), 80.3 (s), 128.6 (d), 134.9 (s), 170.2 (s). HRMS (+ESI): $m / z$ calcd. for $\mathrm{C}_{20} \mathrm{H}_{40} \mathrm{O}_{4} \mathrm{NaSi}(\mathrm{M}+\mathrm{Na})$ 395.2588, found 395.2599.

tert-Butyl (R,E)-5-methyl-6-(((methylthio)carbonothioyl)oxy)-3-((triisopropylsilyl) oxy)hex-4enoate (S5).

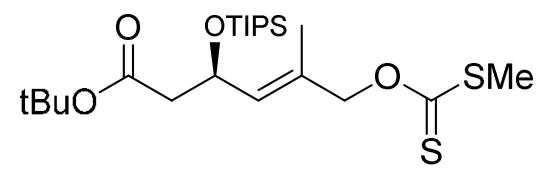

Alcohol $11(1.3 \mathrm{~g}, 3.6 \mathrm{mmol})$ in toluene $(5 \mathrm{~mL})$ was added to a suspension of $\mathrm{NaH}(60 \%$ in mineral oil) $(290 \mathrm{mg}, 7.2 \mathrm{mmol})$ in toluene $(10 \mathrm{~mL})$ at $0{ }^{\circ} \mathrm{C}$ and the mixture was stirred for 30 minutes. After this time, $\mathrm{CS}_{2}$ was added $(1.7 \mathrm{~mL}, 18 \mathrm{mmol})$ and the reaction was stirred for $3 \mathrm{~h}$. Finally MeI (2.2 mL, $18 \mathrm{mmol})$ was added and the reaction was stirred for $1 \mathrm{~h}$ at r.t. The reaction was filtered thorugh celite washing the pad with EtOAc, and the solvent was evaporated. The residue was extracted three times with $\mathrm{CH}_{2} \mathrm{Cl}_{2}$. This organic layer was washed with brine, dried over $\mathrm{MgSO}_{4}$, filtered and the solvent was removed under reduced pressure. Purification by silica gel column chromatography with hexane-EtOAc $(97: 3)$ yielded S5 $(1.48 \mathrm{~g}, 89 \%)$ as an orange oil. $[\alpha]_{\mathrm{D}}$ $=+10.6\left(\mathrm{c} 1.0, \mathrm{CHCl}_{3}\right)$. IR $(\mathrm{KBr}$ film $) \vee 2942,2866,1730,1456,1367,1147,1066,883 \mathrm{~cm}^{-1} .{ }^{1} \mathrm{H}$ NMR (400 MHz, $\left.\mathrm{CDCl}_{3}\right) \delta 1.01-1.06$ (brs, 21H), $1.43(\mathrm{~s}, 9 \mathrm{H}), 1.75(\mathrm{~d}, J=1.4 \mathrm{~Hz}, 3 \mathrm{H}), 2.36$ (dd, $J$ $=14.3,7.0 \mathrm{~Hz}, 1 \mathrm{H}), 2.55(\mathrm{~s}, 3 \mathrm{H}) 2.57(\mathrm{dd}, J=14.3,6.2 \mathrm{~Hz}, 1 \mathrm{H}), 4.90-5.01(\mathrm{~m}, 3 \mathrm{H}), 5.59(\mathrm{dd}, J=$ 8.9, $1.4 \mathrm{~Hz}, 1 \mathrm{H}) .{ }^{13} \mathrm{C} \mathrm{NMR}\left(100.6 \mathrm{MHz}, \mathrm{CDCl}_{3}\right) \delta 12.3$ (d), 14.8 (q), 17.9 (q), 18.9 (q), 28.1 (q), $45.1(\mathrm{t}), 66.4$ (d), 78.4 (t), 80.5 (s), 129.3 (s), 133.6 (d), 169.9 (s), 215.5 (s). HRMS (+ESI): $m / z$ calcd. for $\mathrm{C}_{22} \mathrm{H}_{42} \mathrm{O}_{4} \mathrm{NaS}_{2} \mathrm{Si}(\mathrm{M}+\mathrm{Na})$ 485.2186, found 485.2191. 
tert-Butyl (3R,4RS)-5-methyl-4-(((methylthio)carbonyl)thio)-3-((triisopropylsilyl) oxy)hex-5enoate (12).<smiles>C=C(C)[C@H](SC(=O)SC)[C@@H](CC(=O)OCCC)O[GaH]</smiles>

Xanthate S5 (1.94 g, $4.2 \mathrm{mmol})$ in toluene $(155 \mathrm{~mL})$ was heated at reflux temperature for $16 \mathrm{~h}$. The solvent was evaporated to yield 12 (1.94 g, q.) as a 65(A):35(B) mixture of epimers. IR ( $\mathrm{KBr}$ film) $v 2943,2867,1730,1647,1463,1368,1153,1110,864 \mathrm{~cm}^{-1} .{ }^{1} \mathrm{H}$ NMR $\left(400 \mathrm{MHz}, \mathrm{CDCl}_{3}\right) \delta 1.05-$ $1.11\left(\mathrm{~m}, 21 \mathrm{H}_{\mathrm{A}}+21 \mathrm{H}_{\mathrm{B}}\right), 1.46\left(\mathrm{~s}, 9 \mathrm{H}_{\mathrm{A}}+9 \mathrm{H}_{\mathrm{B}}\right), 1.85-1.86\left(\mathrm{dd}, J=1.5,0.8 \mathrm{~Hz}, 3 \mathrm{H}_{\mathrm{A}}\right), 1.86-1.87(\mathrm{dd}, J$ $\left.=1.5,0.7 \mathrm{~Hz}, 3 \mathrm{H}_{\mathrm{B}}\right), 2.40\left(\mathrm{~s}, 3 \mathrm{H}_{\mathrm{B}}\right), 2.41\left(\mathrm{~s}, 3 \mathrm{H}_{\mathrm{A}}\right), 2.48-2.54\left(\mathrm{~m}, 2 \mathrm{H}_{\mathrm{A}}+1 \mathrm{H}_{\mathrm{B}}\right), 2.70(\mathrm{dd}, J=15.7,8.6$ $\left.\mathrm{Hz}, 1 \mathrm{H}_{\mathrm{B}}\right), 4.40\left(\mathrm{dd}, J=3.4,0.8 \mathrm{~Hz}, 1 \mathrm{H}_{\mathrm{B}}\right), 4.49-4.59\left(\mathrm{~m}, 2 \mathrm{H}_{\mathrm{A}}+1 \mathrm{H}_{\mathrm{B}}\right), 4.93-5.03\left(\mathrm{~m}, 1 \mathrm{H}_{\mathrm{A}}+1 \mathrm{H}_{\mathrm{B}}\right)$, $5.04-5.15\left(\mathrm{~m}, 1 \mathrm{H}_{\mathrm{A}}+1 \mathrm{H}_{\mathrm{B}}\right) .{ }^{13} \mathrm{C} \mathrm{NMR}\left(100.6 \mathrm{MHz}, \mathrm{CDCl}_{3}\right) \delta 12.7$ (d, A), $12.9(\mathrm{~d}, \mathrm{~B}), 13.1$ (q, A+B), $18.1(\mathrm{q}, \mathrm{A}), 18.2$ (q, B), 21.5 (q, B), 21.6 (q, A), 28.0 (q, A+B), 41.8 (t, A), $42.7(\mathrm{t}, \mathrm{A}), 56.4$ (d, A), $57.6(\mathrm{~d}, \mathrm{~B}), 70.5(\mathrm{~d}, \mathrm{~B}), 71.8(\mathrm{~d}, \mathrm{~A}), 81.0(\mathrm{~s}, \mathrm{~A}), 81.0(\mathrm{~s}, \mathrm{~B}), 115.0(\mathrm{t}, \mathrm{B}), 116.7(\mathrm{t}, \mathrm{A}), 141.5(\mathrm{t}, \mathrm{A})$, 142.8 (t, B), 169.8 (s, A+B), 189.1 (s, A+B). HRMS (+ESI): $m / z$ calcd. for $\mathrm{C}_{22} \mathrm{H}_{42} \mathrm{O}_{4} \mathrm{NaS}_{2} \mathrm{Si}(\mathrm{M}+\mathrm{Na})$ 485.2186, found 485.2186 .

tert-Butyl (ZE,R)-5-methyl-6-(tributylstannyl)-3-((triisopropylsilyl)oxy)hex-4-enoate (3).<smiles>[BH3-][OH+]C(C=C(C)CSCCCC)CC(=O)OC(C)(C)C</smiles>

Tributyltin hydride $(0.61 \mathrm{~mL}, 2.25 \mathrm{mmol})$ and AIBN (16 mg, $0.1 \mathrm{mmol})$ were added to a degassed solution of 12 (700 mg, $1.5 \mathrm{mmol})$ in benzene $(40 \mathrm{~mL})$. The reaction was heated at reflux temperature for $6 \mathrm{~h}$ (the colour changes from yellow to grey). Then, the solvent was evaporated and the crude was purified by silica gel column chromatography with hexane-EtOAc $(99: 1)$ and 1\% of Et3N to yield 3 (788 mg, 81\%) as a 7(A):3(B) isomeric double bond mixture. ${ }^{1} \mathrm{H}$ NMR (400 MHz, $\left.\mathrm{CDCl}_{3}\right) \delta 0.79-0.89\left(\mathrm{~m}, 6 \mathrm{H}_{\mathrm{A}}+6 \mathrm{H}_{\mathrm{B}}\right), 0.89\left(\mathrm{t}, J=7.3 \mathrm{~Hz}, 9 \mathrm{H}_{\mathrm{A}}+9 \mathrm{H}_{\mathrm{B}}\right), 1.02-1.06\left(\mathrm{~m}, 21 \mathrm{H}_{\mathrm{A}}+21 \mathrm{H}_{\mathrm{B}}\right)$, $1.25-1.36\left(\mathrm{~m}, 6 \mathrm{H}_{\mathrm{A}}+6 \mathrm{H}_{\mathrm{B}}\right), 1.44\left(\mathrm{~s}, 9 \mathrm{H}_{\mathrm{A}}+9 \mathrm{H}_{\mathrm{B}}\right), 1.45-1.52\left(\mathrm{~m}, 6 \mathrm{H}_{\mathrm{A}}+6 \mathrm{H}_{\mathrm{B}}\right), 1.52-1.55\left(\mathrm{~m}, 1 \mathrm{H}_{\mathrm{A}}\right)$, $1.62\left(\mathrm{~d}, J=1.2 \mathrm{~Hz}, 3 \mathrm{H}_{\mathrm{A}}+3 \mathrm{H}_{\mathrm{B}}\right), 1.66\left(\mathrm{~d}, J=0.8 \mathrm{~Hz}, 1 \mathrm{H}_{\mathrm{B}}\right), 1.70\left(\mathrm{~d}, J=0.8 \mathrm{~Hz}, 1 \mathrm{H}_{\mathrm{B}}\right), 1.97(\mathrm{~d}, J=$ $\left.11.4 \mathrm{~Hz}, 1 \mathrm{H}_{\mathrm{A}}\right), 2.22\left(\mathrm{dd}, J=14.5,3.6 \mathrm{~Hz}, 1 \mathrm{H}_{\mathrm{A}}\right), 2.29\left(\mathrm{dd}, J=14.0,5.8 \mathrm{~Hz}, 1 \mathrm{H}_{\mathrm{B}}\right), 2.38(\mathrm{dd}, J=14.5$, $\left.8.2 \mathrm{~Hz}, 1 \mathrm{H}_{\mathrm{A}}\right), 2.47\left(\mathrm{dd}, J=14.0,7.0 \mathrm{~Hz}, 1 \mathrm{H}_{\mathrm{B}}\right), 4.86-4.93\left(\mathrm{~m}, 1 \mathrm{H}_{\mathrm{A}}+1 \mathrm{H}_{\mathrm{B}}\right), 4.94-4.99\left(\mathrm{~m}, 1 \mathrm{H}_{\mathrm{A}}\right)$, $5.03\left(\mathrm{~m}, 1 \mathrm{H}_{\mathrm{B}}\right) .{ }^{13} \mathrm{C} \mathrm{NMR}\left(100.6 \mathrm{MHz}, \mathrm{CDCl}_{3}\right)$ (cuaternary $\mathrm{C}$ not included) $\delta 9.85(\mathrm{t}, \mathrm{A}+\mathrm{B}), 12.5(\mathrm{~d}$, 
A+B), 13.6 (q, A+B), 15.9 (t, A), 18.1 (q, A+B), 19.0 (q, B), 21.8 (t, B), 26.1 (q, A), 27.3 (t, A+B), 28.1 (q, A+B), 29.2 (t, A+B), 45.5 (t, A), 46.4 (t, B), 67.3 (d, A+B), 124.3 (d, A+B). HRMS (+ESI): $m / z$ calcd. for $\mathrm{C}_{32} \mathrm{H}_{67} \mathrm{O}_{3} \mathrm{SiSn}(\mathrm{M}+\mathrm{H}) 647.3876$, found 647.3882 .

tert-Butyl

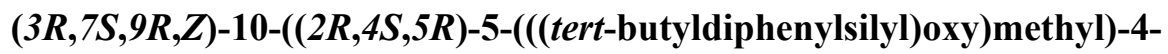

((triethylsilyl)oxy)tetrahydrofuran-2-yl)-7-hydroxy-5,9-dimethyl-3-((triisopropylsilyl)

oxy)dec-4-enoate (13)

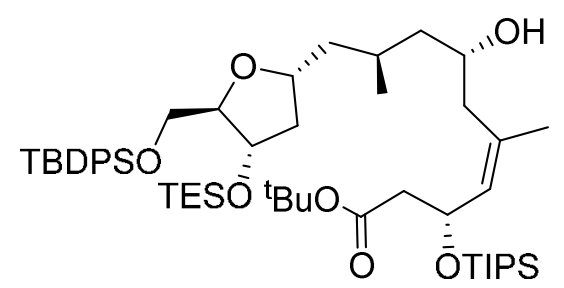

A $1 \mathrm{M} \mathrm{SnCl}_{4}$ solution in $\mathrm{CH}_{2} \mathrm{Cl}_{2}(1.26 \mathrm{~mL}, 1.26 \mathrm{mmol})$ was added slowly to a solution of allylstannane $3(780 \mathrm{mg}, 1.2 \mathrm{mmol})$ in $\mathrm{CH}_{2} \mathrm{Cl}_{2}(10 \mathrm{~mL})$ at $-78{ }^{\circ} \mathrm{C}$. After 10 minutes a solution of aldehyde 2 (580 mg, $1.05 \mathrm{mmol})$ in $\mathrm{CH}_{2} \mathrm{Cl}_{2}(1.5 \mathrm{~mL})$ was added and the reaction was stirred for $2 \mathrm{~h}$ at $-78{ }^{\circ} \mathrm{C}$ and quenched with $\mathrm{NaHCO}_{3}$ saturated solution $(10 \mathrm{~mL})$. The crude was extracted two times with $\mathrm{Et}_{2} \mathrm{O}$ and the organic phase was washed with $10 \% \mathrm{NH}_{3}$, brine and dried over $\mathrm{Na}_{2} \mathrm{SO}_{4}$. The solvent was removed under reduced pressure. Purification by silica gel column chromatography with hexane-EtOAc $(9: 1)$ yielded $13(717 \mathrm{mg}, 75 \%)$ as a single diasteromer. $[\alpha]_{\mathrm{D}}=+5.2$ (c 1.0, $\left.\mathrm{CHCl}_{3}\right)$. IR (KBr film) v 3436 (br), 2955, 2866, 1726, 1470, 1374, 1112, 880, 702 $\mathrm{cm}^{-1}$. ${ }^{1} \mathrm{H}$ NMR $\left(400 \mathrm{MHz}, \mathrm{CDCl}_{3}\right) \delta 0.58(\mathrm{q}, J=8.1 \mathrm{~Hz}, 6 \mathrm{H}), 0.93(\mathrm{t}, J=7.9 \mathrm{~Hz}, 9 \mathrm{H}), 0.97(\mathrm{~d}, J=6.7 \mathrm{~Hz}, 3 \mathrm{H})$, $1.00-1.04(\mathrm{~m}, 21 \mathrm{H}), 1.05(\mathrm{~s}, 9 \mathrm{H}), 1.42(\mathrm{~s}, 9 \mathrm{H}), 1.41-1.43(\mathrm{~m}, 2 \mathrm{H}), 1.54-1.66(\mathrm{~m}, 3 \mathrm{H}), 1.71(\mathrm{~d}, J$ $=1.4 \mathrm{~Hz}, 3 \mathrm{H}), 1.76-1.82(\mathrm{~m}, 1 \mathrm{H}), 1.96(\mathrm{dd}, J=13.7,3.1 \mathrm{~Hz}, 1 \mathrm{H}), 2.25(\mathrm{dt}, J=12.5,6.4 \mathrm{~Hz}, 1 \mathrm{H})$, $2.39(\mathrm{dd}, J=13.7,9.7 \mathrm{~Hz}, 1 \mathrm{H}), 2.45(\mathrm{dd}, J=15.3,6.3 \mathrm{~Hz}, 1 \mathrm{H}), 2.57(\mathrm{dd}, J=15.3,6.3 \mathrm{~Hz}, 1 \mathrm{H})$, $3.65(\mathrm{dd}, J=11.0,3.8 \mathrm{~Hz}, 1 \mathrm{H}), 3.70(\mathrm{dd}, J=11.0,3.8 \mathrm{~Hz}, 1 \mathrm{H}), 3.86(\mathrm{~m}, 2 \mathrm{H}), 4.19$ (dd, $J=7.7,6.2$ $\mathrm{Hz}, 1 \mathrm{H}), 4.49(\mathrm{td}, J=6.4,4.4 \mathrm{~Hz}, 1 \mathrm{H}), 4.87(\mathrm{dt}, J=9.0,6.3 \mathrm{~Hz}, 1 \mathrm{H}), 5.32$ (d, $J=9.0 \mathrm{~Hz}, 1 \mathrm{H}), 7.33$ - $7.44(\mathrm{~m}, 6 \mathrm{H}), 7.65-7.73(\mathrm{~m}, 4 \mathrm{H}) .{ }^{13} \mathrm{C} \mathrm{NMR}\left(100.6 \mathrm{MHz}, \mathrm{CDCl}_{3}\right) \delta 4.7$ (t), 6.8 (q), 12.4 (d), 18.0 (q), $19.2(\mathrm{~s}), 20.9(\mathrm{q}), 23.1(\mathrm{q}), 26.8(\mathrm{q}), 27.5(\mathrm{~d}), 28.1(\mathrm{q}), 41.2(\mathrm{t}), 41.5(\mathrm{t}), 42.8(\mathrm{t}), 45.2(\mathrm{t}), 46.0$ (t), 64.2 (t), 65.9 (d), 66.7 (d), 72.8 (d), 77.2 (d), 80.6 (s), 85.7 (d), 127.6 (d), 127.6 (d), 129.6 (d), 129.6 (d), 131.6 (d), 133.3 (s), 133.4 (s), 133.6 (s), 135.6 (d), 135.7 (d), 171.3 (s). HRMS (+ESI): $m / z$ calcd. for $\mathrm{C}_{52} \mathrm{H}_{90} \mathrm{NaO}_{7} \mathrm{Si}_{3}(\mathrm{M}+\mathrm{Na})$ 933.5887, found 933.5896 . 
$(3 R, 7 S, 9 S, Z)-7-((t e r t-B u t y l d i m e t h y l s i l y l) 0 x y)-10-((2 R, 4 S, 5 R)-5-(($ tert-

butyldiphenylsilyl)oxy)methyl)-4-hydroxytetrahydrofuran-2-yl)-5,9-dimethyl-3-

((triisopropylsilyl)oxy)dec-4-enoic acid (14)

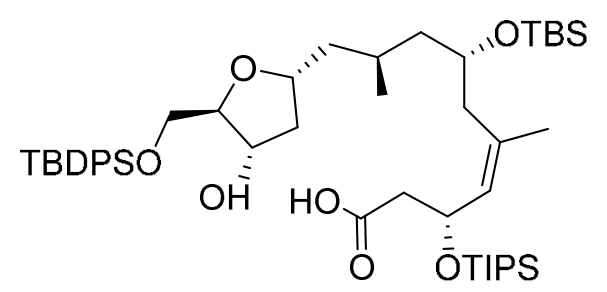

2,6-lutidine $(0.1 \mathrm{~mL}, 0.82 \mathrm{mmol})$ and TBSOTf $(0.05 \mathrm{~mL}, 0.22 \mathrm{mmol})$ were added to a solution of $13(50 \mathrm{mg}, 0.055 \mathrm{mmol})$ in $\mathrm{CH}_{2} \mathrm{Cl}_{2}(3 \mathrm{~mL})$ at $0{ }^{\circ} \mathrm{C}$. The reaction was stirred 30 minutes at r.t. When the protection is quantitative (TLC) TMSOTf $(0.08 \mathrm{~mL}, 0.44$ eq. $)$ was added at $0{ }^{\circ} \mathrm{C}$ and the solution is stirred for 30 minutes at r.t. The reaction was quenched by the addition of saturated $\mathrm{NH}_{4} \mathrm{Cl}$ solution $(3 \mathrm{~mL})$ and the organic phase was washed two times with $0.1 \mathrm{M} \mathrm{HCl}(2 \times 3$ $\mathrm{mL})$. The crude is evaporated and $\mathrm{MeOH}(2 \mathrm{~mL})$ was added. Then PPTS (55 mg, 0.22) was added and the reaction was stirred for 20 minutes. The mixture was evaporated and purification by silica gel column chromatography with hexane-EtOAc (8:2) yielded seco acid 14 (34 mg, 72\%) as a colorless oil. $[\alpha]_{\mathrm{D}}=+3.5\left(\mathrm{c} 1.0, \mathrm{CHCl}_{3}\right)$. IR (KBr film) $\vee 3430($ br), 2930, 2864, 1712, 1463, 1252, $1105,702 \mathrm{~cm}^{-1} .{ }^{1} \mathrm{H}$ NMR $\left(400 \mathrm{MHz}, \mathrm{CDCl}_{3}\right) \delta 0.06(\mathrm{~s}, 3 \mathrm{H}), 0.07(\mathrm{~s}, 3 \mathrm{H}), 0.87(\mathrm{~s}, 9 \mathrm{H}), 0.92(\mathrm{~d}, J=$ $6.6 \mathrm{~Hz}, 3 \mathrm{H}), 1.01-1.07(\mathrm{~m}, 30 \mathrm{H}), 1.22-1.26(\mathrm{~m}, 1 \mathrm{H}), 1.36-1.44(\mathrm{~m}, 1 \mathrm{H}), 1.51-1.56(\mathrm{~m}, 2 \mathrm{H})$, $1.59-1.67(\mathrm{~m}, 2 \mathrm{H}), 1.70(\mathrm{~d}, J=1.3 \mathrm{~Hz}, 3 \mathrm{H}), 1.98(\mathrm{dd}, J=13.5,6.7 \mathrm{~Hz}, 1 \mathrm{H}), 2.30(\mathrm{dd}, J=13.5,6.7$ $\mathrm{Hz}, 1 \mathrm{H}), 2.38$ (dt, $J=13.0,6.7 \mathrm{~Hz}, 1 \mathrm{H}), 2.54$ (dd, $J=14.7,5.6 \mathrm{~Hz}, 1 \mathrm{H}), 2.60$ (dd, $J=14.7,5.6 \mathrm{~Hz}$, 1H), $3.64(\mathrm{dd}, J=10.4,6.3 \mathrm{~Hz}, 1 \mathrm{H}), 3.78$ (dd, $J=10.4,4.3 \mathrm{~Hz}, 1 \mathrm{H}), 3.85-3.91(\mathrm{~m}, 2 \mathrm{H}), 4.12$ $4.20(\mathrm{~m}, 1 \mathrm{H}), 4.42(\mathrm{td}, J=6.3,4.3 \mathrm{~Hz}, 1 \mathrm{H}), 4.88(\mathrm{dt}, J=8.5,5.6 \mathrm{~Hz}, 1 \mathrm{H}), 5.31(\mathrm{~d}, J=8.5 \mathrm{~Hz}, 1 \mathrm{H})$, $7.35-7.46(\mathrm{~m}, 6 \mathrm{H}), 7.63-7.69(\mathrm{~m}, 4 \mathrm{H}) .{ }^{13} \mathrm{C} \mathrm{NMR}\left(100.6 \mathrm{MHz}, \mathrm{CDCl}_{3}\right) \delta-4.5$ (q), -3.9 (q), 12.3 (d), 17.9 (d), 18.0 (q), 18.0 (s), 19.2 (s), 20.9 (q), 24.2 (q), 25.9 (q), 26.8 (q), 27.3 (d), 40.8 (t), 40.9 $(\mathrm{t}), 43.3(\mathrm{t}), 43.6(\mathrm{t}), 45.0(\mathrm{t}), 65.0(\mathrm{t}), 66.8(\mathrm{~d}), 69.4(\mathrm{~d}), 74.6(\mathrm{~d}), 76.9(\mathrm{~d}), 84.3(\mathrm{~d}), 127.7(\mathrm{~d}), 127.8$ (d), 129.8 (d), 129.8 (d), 133.1 (s), 134.2 (s), 135.5 (d), 135.6 (d), 173.2 (s). HRMS (+ESI): m/z calcd. for $\mathrm{C}_{48} \mathrm{H}_{82} \mathrm{NaO}_{7} \mathrm{Si}_{3}(\mathrm{M}+\mathrm{Na})$ 877.5261, found 877.5278. 
$(1 S, 5 R, 9 S, 11 S, 13 R, 15 R, Z)-9-(($ tert-Butyldimethylsilyl)oxy)-15-((tert-

butyldiphenylsilyl)oxy)methyl)-7,11-dimethyl-5-((triisopropylsilyl)oxy)-2,14-

dioxabicyclo[11.2.1]hexadec-6-en-3-one (15)

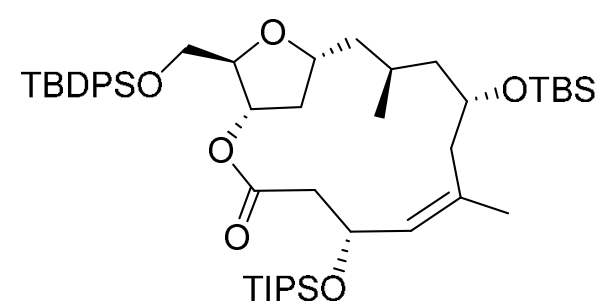

A solution of hydroxyacid $14(223 \mathrm{mg}, 0.26 \mathrm{mmol})$ in toluene $(60 \mathrm{~mL})$ was added during $24 \mathrm{~h}$ using a syringe pump over a solution of DMAP (230 mg, $1.88 \mathrm{mmol})$ and MNBA (313 mg, $0.91 \mathrm{mmol})$ in toluene $(220 \mathrm{~mL})$ at r.t. The reaction mixture was stirred 2 hours after addition finished and was quenched with a saturated $\mathrm{NH}_{4} \mathrm{Cl}$ solution $(45 \mathrm{~mL})$. The crude was extracted with $\mathrm{CH}_{2} \mathrm{Cl}_{2}$ and the organic phase was dried over $\mathrm{Na}_{2} \mathrm{SO}_{4}$ and the solvent was removed under reduced pressure. Purification by silica gel column chromatography with hexane-EtOAc (95:5) yielded macrolactone $15(145 \mathrm{mg}, 67 \%)$ as a colorless oil. $[\alpha]_{\mathrm{D}}=-11.2\left(\mathrm{c} 1.0, \mathrm{CHCl}_{3}\right) . \mathrm{IR}(\mathrm{KBr}$ film $) v$ 2929, 2864, 1742, 1450, 1112, 1063, 926, $702 \mathrm{~cm}^{-1} .{ }^{1} \mathrm{H}$ NMR (400 MHz, $\left.\mathrm{CDCl}_{3}\right) \delta 0.02(\mathrm{~s}, 3 \mathrm{H})$, $0.06(\mathrm{~s}, 3 \mathrm{H}), 0.84(\mathrm{~s}, 9 \mathrm{H}), 0.93(\mathrm{~d}, J=6.2 \mathrm{~Hz}, 3 \mathrm{H}), 1.02-1.07(\mathrm{~m}, 30 \mathrm{H}), 1.30-1.33(\mathrm{~m}, 1 \mathrm{H}), 1.41$ $-1.45(\mathrm{~m}, 1 \mathrm{H}), 1.59-1.68(\mathrm{~m}, 2 \mathrm{H}), 1.72$ (d, $J=1.4 \mathrm{~Hz}, 3 \mathrm{H}), 1.91-1.99$ (m, 1H), 2.06 (d, $J=13.2$ $\mathrm{Hz}, 1 \mathrm{H}), 2.25$ (ddd, $J=13.2,8.0,6.2 \mathrm{~Hz}, 1 \mathrm{H}), 2.35$ (ddd, $J=14.1,11.0,3.1 \mathrm{~Hz}, 1 \mathrm{H}), 2.53$ (dd, $J=$ 14.1, $7.8 \mathrm{~Hz}, 1 \mathrm{H}), 2.68(\mathrm{dd}, J=13.6,9.7 \mathrm{~Hz}, 1 \mathrm{H}), 2.75(\mathrm{dd}, J=14.1,2.8 \mathrm{~Hz}, 1 \mathrm{H}), 3.70(\mathrm{dd}, J=$ 10.9, $3.4 \mathrm{~Hz}, 1 \mathrm{H}), 3.86(\mathrm{dd}, J=10.9,3.1 \mathrm{~Hz}, 1 \mathrm{H}), 4.01-4.08(\mathrm{~m}, 2 \mathrm{H}), 4.43-4.52(\mathrm{~m}, 1 \mathrm{H}), 5.11$ $(\mathrm{td}, J=7.4,2.8 \mathrm{~Hz}, 1 \mathrm{H}), 5.26(\mathrm{~d}, J=5.7 \mathrm{~Hz}, 1 \mathrm{H}), 5.43(\mathrm{~d}, J=7.8 \mathrm{~Hz}, 1 \mathrm{H}), 7.34-7.43(\mathrm{~m}, 6 \mathrm{H})$, $7.63-7.71(\mathrm{~m}, 4 \mathrm{H}) .{ }^{13} \mathrm{C} \mathrm{NMR}\left(100.6 \mathrm{MHz}, \mathrm{CDCl}_{3}\right) \delta-4.4$ (q), -4.1 (q), 12.3 (d), 17.9 (q), 18.0 (q), 18.1 (s), 19.3 (s), 20.9 (q), 23.3 (q), 25.9 (q), 26.7 (d), 26.8 (q), 34.1 (t), 38.5 (t), 41.2 (t), 46.1 (t), $47.0(\mathrm{t}), 65.0$ (t), 68.3 (d), 70.7 (d), 77.4 (d), 78.6 (d), 84.7 (d), 127.7 (d), 127.7 (d), 129.6 (d), 129.7 (d), 132.6 (d), 132.9 (s), 133.3 (s), 133.4 (s), 135.6 (d), 135.6 (d), 171.0(s) . HRMS (+ESI): m/z calcd. for $\mathrm{C}_{48} \mathrm{H}_{84} \mathrm{NO}_{6} \mathrm{Si}_{3}\left(\mathrm{M}+\mathrm{NH}_{4}\right)$ 854.5601, found 854.5601. 
(1S,5R,9S,11S,13R,15R,Z)-9-((tert-Butyldimethylsilyl)oxy)-15-(hydroxymethyl)-7,11-dimethyl5-((triisopropylsilyl)oxy)-2,14-dioxabicyclo[11.2.1] hexadec-6-en-3-one (S6)

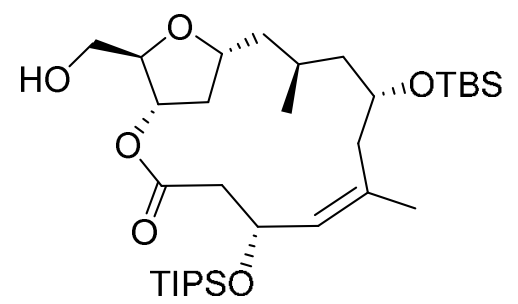

A buffered TBAF/AcOH 1:1 solution (1.44 mL, $0.22 \mathrm{mmol}$ of TBAF) was added to a solution of lactone $15(15 \mathrm{mg}, 0.018 \mathrm{mmol})$ in THF $(1 \mathrm{~mL})$ and the mixture is stirred $16 \mathrm{~h}$ at $40{ }^{\circ} \mathrm{C}$. The reaction was quenched with a saturated $\mathrm{NaHCO}_{3}$ solution $(1 \mathrm{~mL})$. The crude was extracted twice with EtOAc and the organic phase was dried over $\mathrm{Na}_{2} \mathrm{SO}_{4}$ and the solvent was removed under reduced pressure. Purification by silica gel column chromatography with hexane-EtOAc (95:5) yielded alcohol $\mathbf{S 6}(8 \mathrm{mg}, 72 \%)$ as a colorless oil. ${ }^{1} \mathrm{H}$ NMR $\left(400 \mathrm{MHz}, \mathrm{CDCl}_{3}\right) \delta 0.01(\mathrm{~s}, 3 \mathrm{H}), 0.06$ (s, 3H), $0.84(\mathrm{~s}, 9 \mathrm{H}), 0.91(\mathrm{~d}, J=6.4 \mathrm{~Hz}, 3 \mathrm{H}), 1.02(\mathrm{~s}, 21 \mathrm{H}), 1.25-1.30(\mathrm{~m}, 1 \mathrm{H}), 1.41-1.48(\mathrm{~m}$, $1 \mathrm{H}), 1.57-1.65(\mathrm{~m}, 1 \mathrm{H}), 1.70(\mathrm{~d}, J=1.4 \mathrm{~Hz}, 3 \mathrm{H}), 1.71-1.76(\mathrm{~m}, 1 \mathrm{H}), 1.84(\mathrm{~d}, J=13.8 \mathrm{~Hz}, 1 \mathrm{H})$, $2.07-2.12(\mathrm{~m}, 2 \mathrm{H}), 2.44-2.54(\mathrm{~m}, 2 \mathrm{H}), 2.75-2.86(\mathrm{~m}, 2 \mathrm{H}), 3.65-3.76(\mathrm{~m}, 2 \mathrm{H}), 4.00(\mathrm{dt}, J=$ 4.2, $2.2 \mathrm{~Hz}, 1 \mathrm{H}), 4.08-4.15(\mathrm{~m}, 1 \mathrm{H}), 4.42-4.50(\mathrm{~m}, 1 \mathrm{H}), 4.99-5.03(\mathrm{~m}, 1 \mathrm{H}), 5.15(\mathrm{t}, J=7.6 \mathrm{~Hz}$, $1 \mathrm{H}), 5.45(\mathrm{dt}, J=7.8,1.4 \mathrm{~Hz}, 1 \mathrm{H}) .{ }^{13} \mathrm{C} \mathrm{NMR}\left(100.6 \mathrm{MHz}, \mathrm{CDCl}_{3}\right) \delta-4.4$ (q), -4.1 (q), $12.2(\mathrm{~d}), 17.9$ (q), 17.9 (q), 18.1 (s), 20.7 (q), 22.6 (q), 25.9 (q), 26.6 (d), 33.8 (t), 38.0 (t), $40.4(\mathrm{t}), 45.8(\mathrm{t}), 47.2$ (t), 63.4 (t), 68.4 (d), 70.1 (d), 78.7 (d), 84.7 (d), 132.5 (s), 133.0 (d), 171.5 (s). HRMS (+ESI): m/z calcd. for $\mathrm{C}_{32} \mathrm{H}_{62} \mathrm{NaO}_{6} \mathrm{Si}_{2}(\mathrm{M}+\mathrm{Na})$ 621.3977, found 621.3961 .

\section{(1S,5R,9S,11S,13R,15S,Z)-9-((tert-Butyldimethylsilyl)oxy)-7,11-dimethyl-3-oxo-5-}

((triisopropylsilyl)oxy)-2,14-dioxabicyclo[11.2.1]hexadec-6-ene-15-carbaldehyde (16)

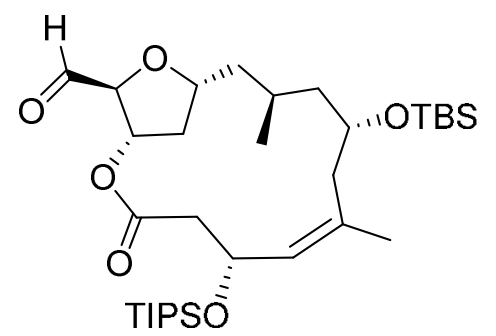

Dess-Martin periodinane (28 mg, $0.064 \mathrm{mmol}$ ) was added to a suspension of alcohol S6 (32 $\mathrm{mg}, 0.054 \mathrm{mmol})$ and $\mathrm{NaHCO}_{3}(11 \mathrm{mg}, 0.128 \mathrm{mmol})$ in $\mathrm{CH}_{2} \mathrm{Cl}_{2}(2 \mathrm{~mL})$ and the mixture was stirred for 45 minutes. The reaction mixture was diluted with sat. $\mathrm{Na}_{2} \mathrm{~S}_{2} \mathrm{O}_{3}$ and sat. $\mathrm{NaHCO}_{3}$ and the 
resulting solution was extracted with $\mathrm{Et}_{2} \mathrm{O}$. The organic extracts were dried over $\mathrm{MgSO}_{4}$, filtered and concentrated under reduced pressure. Purification by silica gel column chromatography with hexane-EtOAc (9.1) yielded 2 (31 mg, quant.) as a colorless oil. IR ( $\mathrm{KBr}$ film) v 2958, 2921, 1737, 1460, 1378, 1057, 831 $\mathrm{cm}^{-1} .{ }^{1} \mathrm{H}$ NMR $\left(400 \mathrm{MHz}, \mathrm{CDCl}_{3}\right) \delta 0.02(\mathrm{~s}, 3 \mathrm{H}), 0.06(\mathrm{~s}, 3 \mathrm{H}), 0.84(\mathrm{~s}, 9 \mathrm{H})$, $0.93(\mathrm{~d}, J=6.3 \mathrm{~Hz}, 3 \mathrm{H}), 1.03(\mathrm{~s}, 21 \mathrm{H}), 1.37-1.41(\mathrm{~m}, 1 \mathrm{H}), 1.42-1.46(\mathrm{~m}, 1 \mathrm{H}), 1.62(\mathrm{dd}, J=9.4$, $4.6 \mathrm{~Hz}, 1 \mathrm{H}), 1.65-1.69(\mathrm{~m}, 1 \mathrm{H}), 1.71(\mathrm{~d}, J=1.4 \mathrm{~Hz}, 3 \mathrm{H}), 1.91(\mathrm{dd}, J=13.6,3.3 \mathrm{~Hz}, 1 \mathrm{H}), 1.97-$ $2.02(\mathrm{~m}, 1 \mathrm{H}), 2.13(\mathrm{~d}, J=14.3 \mathrm{~Hz}, 1 \mathrm{H}), 2.41(\mathrm{ddd}, J=14.2,11.0,3.4 \mathrm{~Hz}, 1 \mathrm{H}), 2.57(\mathrm{dd}, J=14.1$, $7.2 \mathrm{~Hz}, 1 \mathrm{H}), 2.68$ (dd, $J=13.6,9.9 \mathrm{~Hz}, 1 \mathrm{H}), 2.79$ (dd, $J=14.1,2.8 \mathrm{~Hz}, 1 \mathrm{H}), 4.03-4.08$ (m, 1H), $4.51(\mathrm{~d}, J=1.2 \mathrm{~Hz}, 1 \mathrm{H}), 4.55-4.63(\mathrm{~m}, 1 \mathrm{H}), 5.09(\mathrm{td}, J=7.4,2.9 \mathrm{~Hz}, 1 \mathrm{H}), 5.27(\mathrm{dd}, J=5.5,1.2$ $\mathrm{Hz}, 1 \mathrm{H}), 5.42(\mathrm{dt}, J=7.8,1.4 \mathrm{~Hz}, 1 \mathrm{H}), 9.71(\mathrm{~s}, 1 \mathrm{H}) .{ }^{13} \mathrm{C} \mathrm{NMR}\left(100.6 \mathrm{MHz}, \mathrm{CDCl}_{3}\right) \delta-4.4(\mathrm{q}),-4.1$ (q), 12.3 (d), 17.9 (q), 17.9 (q), 18.1 (s), 20.8 (q), 23.1 (q), 25.8 (q), 26.6 (d), 33.0 (t), 38.4 (t), 40.9 (t), $45.8(\mathrm{t}), 46.7$ (t), 68.1 (d), 70.5 (d), 74.7 (d), 79.8 (d), 88.4 (d), 132.5 (d), 133.0 (s), 170.8 (s), 198.5 (d). HRMS (+ESI): $m / z$ calcd. for $\mathrm{C}_{32} \mathrm{H}_{60} \mathrm{NaO}_{6} \mathrm{Si}_{2}(\mathrm{M}+\mathrm{Na}) 619.3821$, found 619.3833 .

\section{(1S,5R,9S,11R,13R,15R,Z)-5,9-Dihydroxy-15-(hydroxymethyl)-7,11-dimethyl-2,14-}

\section{dioxabicyclo[11.2.1]hexadec-6-en-3-one (1)}

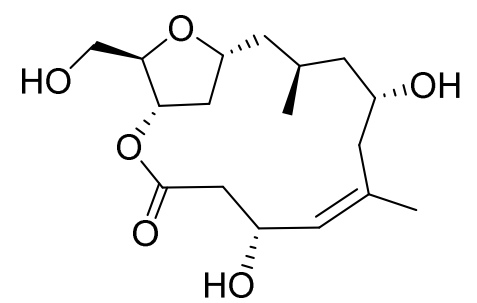

A buffered TBAF/AcOH 1:1 solution (1.3 mL, $0.81 \mathrm{mmol}$ of TBAF) was added to a solution of lactone $15(45 \mathrm{mg}, 0.054 \mathrm{mmol})$ in 1,4-dioxane $(3 \mathrm{~mL})$ and the mixture is stirred $36 \mathrm{~h}$ at $90{ }^{\circ} \mathrm{C}$. The reaction was quenched with a saturated $\mathrm{NaHCO}_{3}$ solution $(1 \mathrm{~mL})$. The crude was extracted twice with EtOAc and the organic phase was dried over $\mathrm{Na}_{2} \mathrm{SO}_{4}$ and the solvent was removed under reduced pressure. Purification by silica gel column chromatography with EtOAc:MeOH (99:1) yielded triol 1 (9.6 mg, 54\%) as a colorless oil. IR (KBr film) v 3387(br), 2947, 2918, 1728, 1459, $1051 \mathrm{~cm}^{-1} .{ }^{1} \mathrm{H}$ NMR $\left(400 \mathrm{MHz}, \mathrm{CDCl}_{3}\right) \delta 0.96(\mathrm{~d}, J=6.0 \mathrm{~Hz}, 3 \mathrm{H}), 1.34-$ $1.46(\mathrm{~m}, 2 \mathrm{H}), 1.59-1.68(\mathrm{~m}, 2 \mathrm{H}), 1.80$ (d, $J=1.5 \mathrm{~Hz}, 3 \mathrm{H}), 2.00-2.17$ (m, 4H), $2.60-2.65(\mathrm{~m}$, 2H), $2.74(\mathrm{dd}, J=13.4,7.7 \mathrm{~Hz}, 1 \mathrm{H}), 3.62-3.71(\mathrm{~m}, 2 \mathrm{H}), 3.97(\mathrm{td}, J=9.9,7.7,4.4 \mathrm{~Hz}, 1 \mathrm{H}), 4.11$ $(\mathrm{td}, J=4.5,1.5 \mathrm{~Hz}, 1 \mathrm{H}), 4.35-4.44(\mathrm{~m}, 1 \mathrm{H}), 4.84(\mathrm{dt}, J=8.7,5.8 \mathrm{~Hz}, 1 \mathrm{H}), 5.14(\mathrm{dd}, J=5.9,1.5$ $\mathrm{Hz}, 1 \mathrm{H}), 5.49$ (dd, $J=8.7,1.5 \mathrm{~Hz}, 1 \mathrm{H}) .{ }^{13} \mathrm{C} \mathrm{NMR}\left(100.6 \mathrm{MHz}, \mathrm{CDCl}_{3}\right) \delta 20.6$ (q), 24.9 (q), 27.0 (d), $34.8(\mathrm{t}), 39.0(\mathrm{t}), 41.0(\mathrm{t}), 44.3(\mathrm{t}), 44.9(\mathrm{t}), 63.3(\mathrm{t}), 65.9(\mathrm{~d}), 69.9(\mathrm{~d}), 77.7(\mathrm{~d}), 78.3(\mathrm{~d}), 84.1(\mathrm{~d})$, 
129.9 (d), 137.1 (s), 171.5 (s). HRMS (+ESI): $m / z$ calcd. for $\mathrm{C}_{17} \mathrm{H}_{28} \mathrm{NaO}_{6}(\mathrm{M}+\mathrm{Na})$ 351.1778, found 351.1789 .

\section{NOE 1D experiment of $7 \mathrm{a}$}

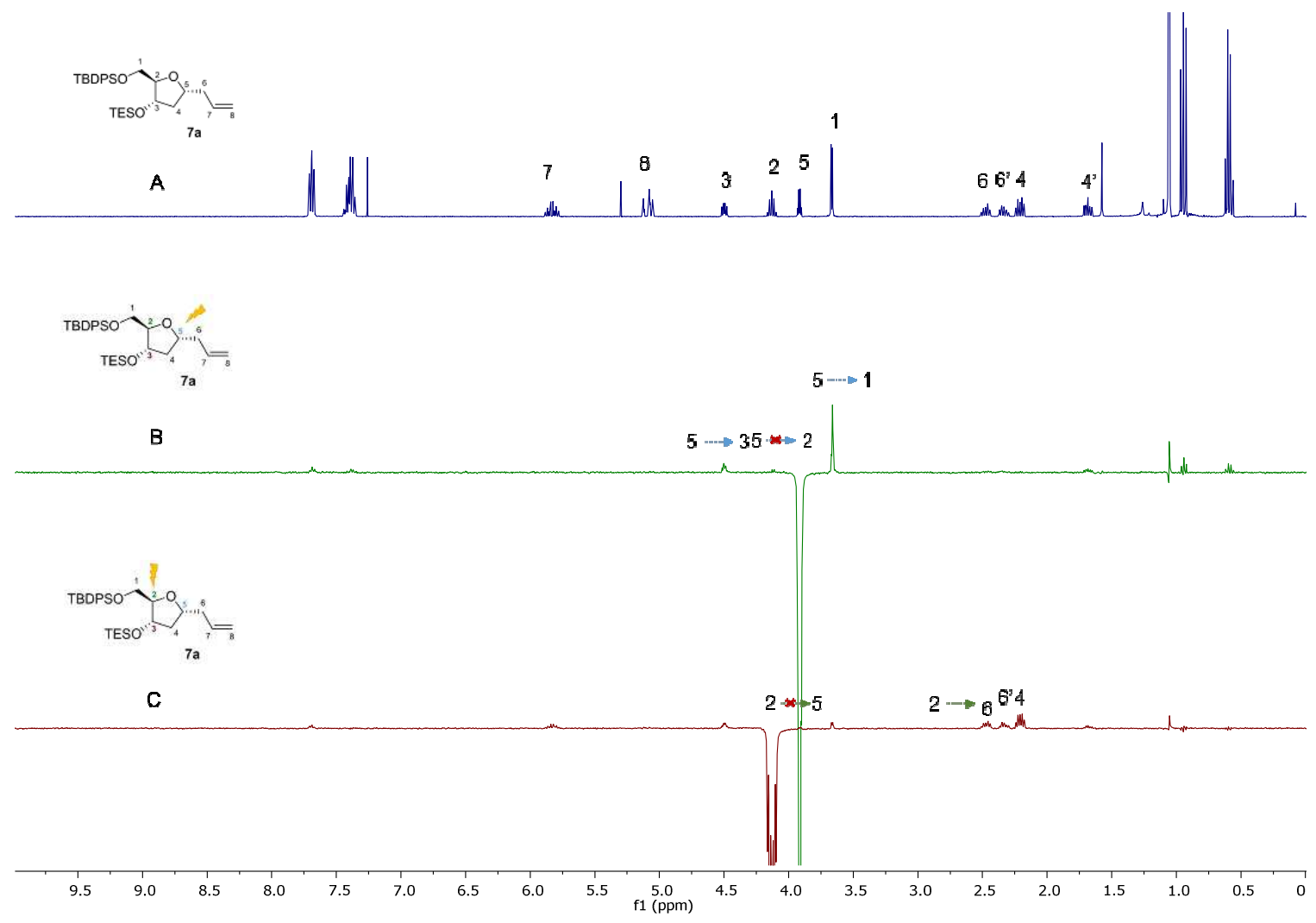

Scheme S1. A) H-NMR of compound 7a; B) irradiation to proton in position $5\left(\mathrm{H}^{5}\right)$; C) irradiation to proton in position $2\left(\mathrm{H}^{2}\right)$.

Absolute configuration of stereocenters at positions 2 and 3 are determined by the used starting material (2-D-deoxyribose). When proton 5 was irradiated, no NOE correlation with $\mathrm{H}^{2}$ was osbserved, confirming that susbtituents in position 2 and 5 are in anti- relative position. Therefore the created stereocenter in $\mathrm{C} 5$ has $\mathrm{R}$ stereochemistry. Irradiation of $\mathrm{H}^{2}$ has no correlation with $\mathrm{H}^{5}$ (as expected) but it has correlation with the two hydrogens in position 6 (the substituent in $\mathrm{C}^{5}$ is in syn- relation with $\mathrm{H}^{2}$ ) and with $\mathrm{H}^{4}$ (the hydrogen in position 4 located in syn to $\mathrm{H}^{2}$ ). 


\section{Mosher's derivatization of compound 13}

tert-Butyl

$(3 R, 7 S, 9 S, Z)-10-((2 R, 4 S, 5 R)-5-(($ tert-butyldiphenylsilyl)oxy)methyl)-4-

((triethylsilyl)oxy)tetrahydrofuran-2-yl)-7-((S)-2-methoxy-2-phenylacetoxy)-5,9-dimethyl-3-

((triisopropylsilyl)oxy)dec-4-enoate (S7)

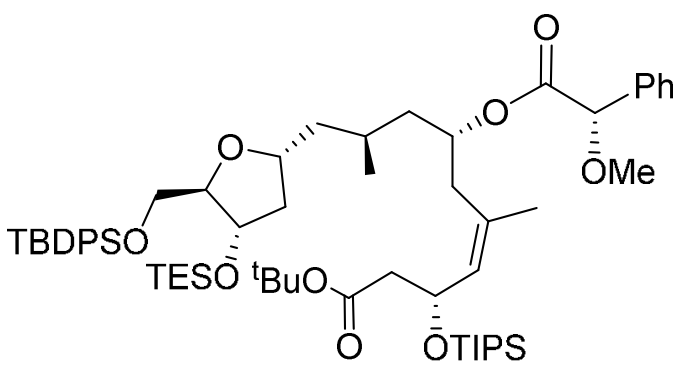

(S)- $\alpha$-Methoxyphenylacetic acid (31 $\mathrm{mg}, 0.19 \mathrm{mmol})$ and $\mathrm{EDC} \cdot \mathrm{HCl}(30 \mathrm{mg}, 0.19 \mathrm{mmol})$ were added to a solution of alcohol $13(25 \mathrm{mg}, 0.027 \mathrm{mmol})$ in THF $(1 \mathrm{~mL})$, then DMAP $(0.3 \mathrm{mg}, 0.003$ mmol) was added and the solution was stirred for $120 \mathrm{~min}$. The solution was filtered, poured into $\mathrm{Et}_{2} \mathrm{O}$ and washed with with aqueous $0.2 \mathrm{M} \mathrm{HCl}$ and sat. $\mathrm{NaHCO}_{3}$. The organic residue was dried over $\mathrm{MgSO}_{4}$, filtered and the solvent was removed under reduced pressure. Purification by silica gel column chromatography with hexane-EtOAc (90:10) yielded $\mathbf{S 7}(20 \mathrm{mg}, 70 \%)$ as a colorless oil. ${ }^{1} \mathrm{H}$ NMR (400 MHz, $\left.\mathrm{CDCl}_{3}\right) \delta{ }^{1} \mathrm{H} \mathrm{NMR}(400 \mathrm{MHz}$, Chloroform- $d$ ) $\delta 0.58$ (q, $J=7.9 \mathrm{~Hz}, 6 \mathrm{H}), 0.91(\mathrm{~m}$, 3H), $0.92(\mathrm{~m}, 1 \mathrm{H}), 0.94(\mathrm{t}, J=7.9 \mathrm{~Hz}, 9 \mathrm{H}), 0.98-1.04(\mathrm{~m}, 21 \mathrm{H}), 1.05(\mathrm{~s}, 9 \mathrm{H}), 1.43$ (s, 9H), 1.48 $(\mathrm{d}, J=1.4 \mathrm{~Hz}, 3 \mathrm{H}), 1.49-1.56(\mathrm{~m}, 4 \mathrm{H}), 2.05-2.34(\mathrm{~m}, 6 \mathrm{H}), 3.35(\mathrm{~s}, 3 \mathrm{H}), 3.60-3.72(\mathrm{~m}, 2 \mathrm{H})$, $3.82(\mathrm{q}, J=3.9 \mathrm{~Hz}, 1 \mathrm{H}), 3.98-4.07(\mathrm{~m}, 1 \mathrm{H}), 4.45(\mathrm{td}, J=6.5,4.6 \mathrm{~Hz}, 1 \mathrm{H}), 4.86(\mathrm{td}, J=8.1,4.4$ $\mathrm{Hz}, 1 \mathrm{H}), 5.16-5.23(\mathrm{~m}, 2 \mathrm{H}), 7.30-7.46(\mathrm{~m}, 11 \mathrm{H}), 7.66-7.72(\mathrm{~m}, 4 \mathrm{H})$. HRMS (+ESI): $\mathrm{m} / z$ calcd. for $\mathrm{C}_{61} \mathrm{H}_{98} \mathrm{NaO}_{9} \mathrm{Si}_{3}(\mathrm{M}+\mathrm{Na})$ 1081.6411, found 1081.6407.

tert-Butyl (3R,7S,9S,Z)-10-((2R,4S,5R)-5-(((tert-butyldiphenylsilyl)oxy)methyl)-4-

((triethylsilyl)oxy)tetrahydrofuran-2-yl)-7-((R)-2-methoxy-2-phenylacetoxy)-5,9-dimethyl-3((triisopropylsilyl)oxy)dec-4-enoate (S8) 


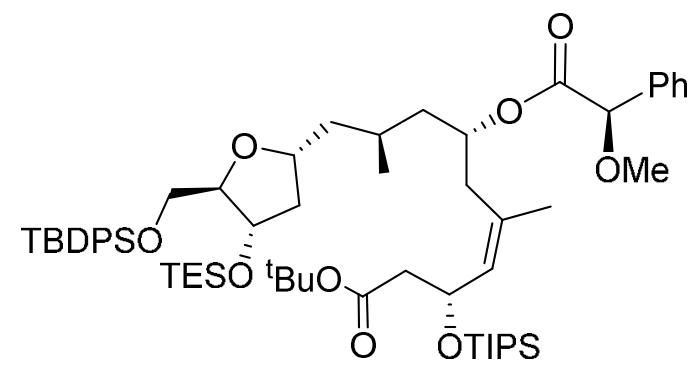

(R)- $\alpha$-Methoxyphenylacetic acid (31 $\mathrm{mg}, 0.19 \mathrm{mmol})$ and $\mathrm{EDC} \cdot \mathrm{HCl}(30 \mathrm{mg}, 0.19 \mathrm{mmol})$ were added to a solution of alcohol $13(25 \mathrm{mg}, 0.027 \mathrm{mmol})$ in THF $(1 \mathrm{~mL})$, then DMAP $(0.3 \mathrm{mg}, 0.003$ mmol) was added and the solution was stirred for $120 \mathrm{~min}$. The solution was filtered, poured into $\mathrm{Et}_{2} \mathrm{O}$ and washed with with aqueous $0.2 \mathrm{M} \mathrm{HCl}$ and sat. $\mathrm{NaHCO}_{3}$. The organic residue was dried over $\mathrm{MgSO}_{4}$, filtered and the solvent was removed under reduced pressure. Purification by silica gel column chromatography with hexane-EtOAc $(90: 10)$ yielded $\mathbf{S 8}(23 \mathrm{mg}, 80 \%)$ as a colorless oil. ${ }^{1} \mathrm{H}$ NMR (400 MHz, $\left.\mathrm{CDCl}_{3}\right) 0.58$ (q, $\left.J=7.9 \mathrm{~Hz}, 6 \mathrm{H}\right), 0.70(\mathrm{~d}, J=6.6 \mathrm{~Hz}, 3 \mathrm{H}), 0.94$ (t, $\left.J=7.9 \mathrm{~Hz}, 9 \mathrm{H}\right)$, $0.94(\mathrm{~m}, 1 \mathrm{H}), 0.99-1.07(\mathrm{~m}, 30 \mathrm{H}), 1.22-1.41(\mathrm{~m}, 4 \mathrm{H}), 1.41-1.44(\mathrm{~m}, 1 \mathrm{H}), 1.46(\mathrm{~s}, 9 \mathrm{H}), 1.67$ (d, $J=1.4 \mathrm{~Hz}, 3 \mathrm{H}), 2.00-2.15(\mathrm{~m}, 2 \mathrm{H}), 2.33-2.50(\mathrm{~m}, 3 \mathrm{H}), 3.36(\mathrm{~s}, 3 \mathrm{H}), 3.58-3.69(\mathrm{~m}, 2 \mathrm{H}), 3.78$ $(\mathrm{q}, J=3.9 \mathrm{~Hz}, 1 \mathrm{H}), 3.81-3.89(\mathrm{~m}, 1 \mathrm{H}), 4.42(\mathrm{td}, J=6.5,4.7 \mathrm{~Hz}, 1 \mathrm{H}), 4.66(\mathrm{~s}, 1 \mathrm{H}), 4.95(\mathrm{td}, J=$ 8.2, $4.1 \mathrm{~Hz}, 1 \mathrm{H}), 5.18$ (dt, $J=8.4,3.8 \mathrm{~Hz}, 1 \mathrm{H}), 5.33$ (dd, $J=8.7,1.6 \mathrm{~Hz}, 1 \mathrm{H}), 7.24-7.44(\mathrm{~m}, 11 \mathrm{H})$, 7.66 - $7.71(\mathrm{~m}, 4 \mathrm{H})$. HRMS (+ESI): $\mathrm{m} / z$ calcd. for $\mathrm{C}_{61} \mathrm{H}_{98} \mathrm{NaO}_{9} \mathrm{Si}_{3}(\mathrm{M}+\mathrm{Na})$ 1081.6411, found 1081.6400 .

\section{Absolute configuration determination:}

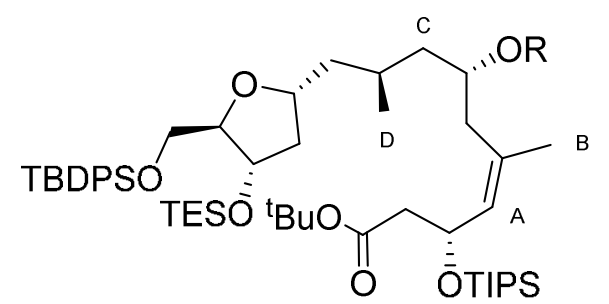

\begin{tabular}{c|c|c|c|c} 
& $\delta \mathbf{H}_{\mathrm{A}}$ & $\delta \mathbf{H}_{\mathrm{B}}$ & $\delta \mathbf{H}_{\mathrm{C}}$ & $\delta \mathbf{H}_{\mathrm{D}}$ \\
\hline $\mathbf{R}=(\boldsymbol{R})-\mathrm{MPA}$ & $\mathbf{5 . 3 3}$ & $\mathbf{1 . 6 7}$ & 1.31 & $\mathbf{0 . 7 0}$ \\
\hline $\mathbf{R}=(S)-\mathrm{MPA}$ & 5.19 & 1.49 & 1.47 & $\mathbf{0 . 9 1}$ \\
\hline$\Delta^{\mathrm{RS}}$ & $\mathbf{0 . 1 4}$ & $\mathbf{0 . 1 8}$ & $\mathbf{- 0 . 2 1}$ & $\mathbf{- 0 . 2 1}$
\end{tabular}




\section{Bibliography}

(1) Teng, K.; Marquez, V. E.; Mihe, G. W. a; Barchi, J. J.; Kazanietz, M. G.; Lewin, N. E.; Blumberg, P. M.; Abushanabg, E. J. Am. Chem. Soc. 1992, 114 (3), 1059.

(2) Lorente, A.; Gil, A.; Fernández, R.; Cuevas, C.; Albericio, F.; Álvarez, M. Chem. - A Eur. Journal. 2015, 21, 150.

(3) Boisse, T.; Rigo, B.; Millet, R.; Hénichart, J.-P. Tetrahedron. 2007, 63, 10511. 
NMR data of compounds 5-S8

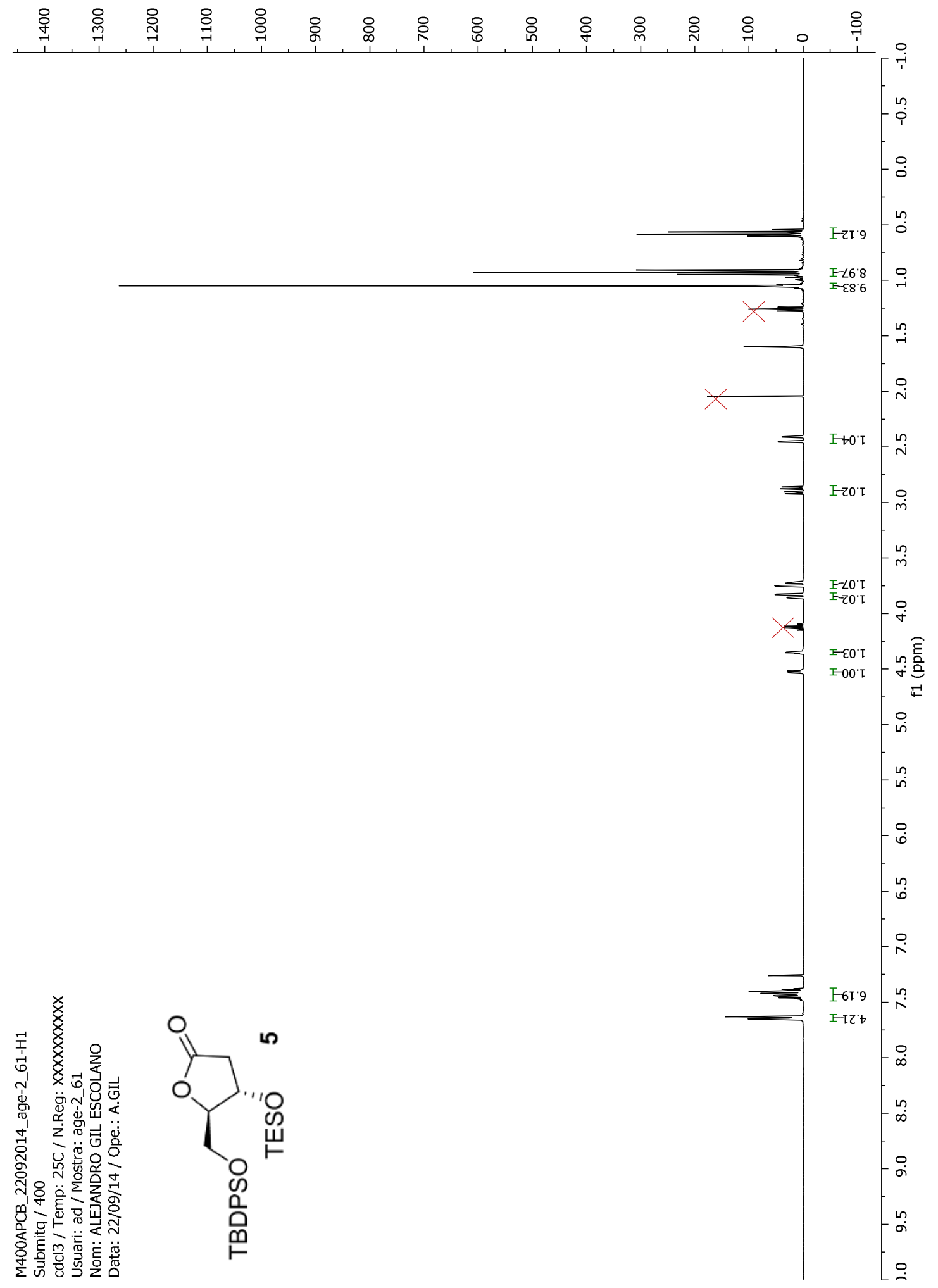




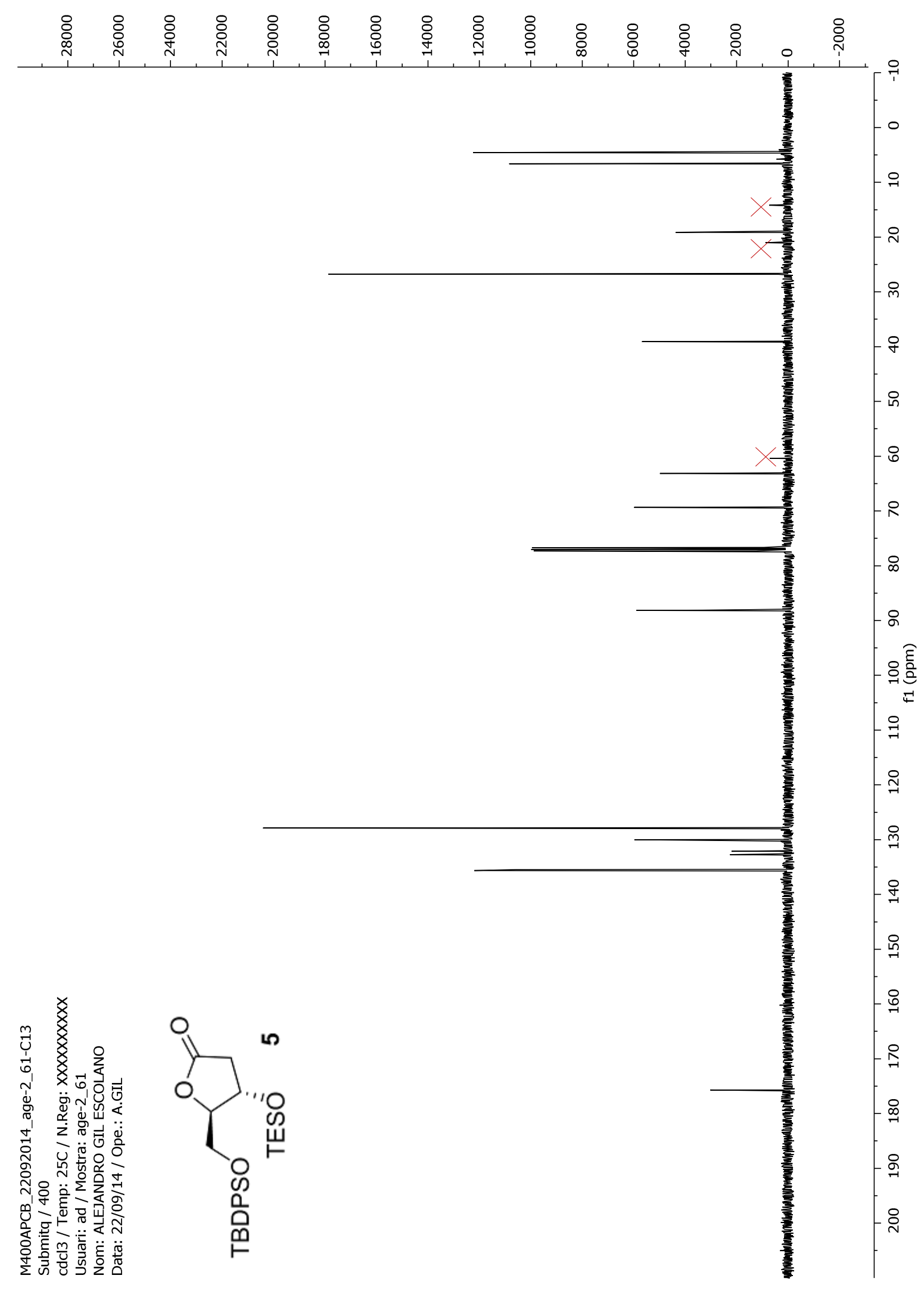


(udd) If

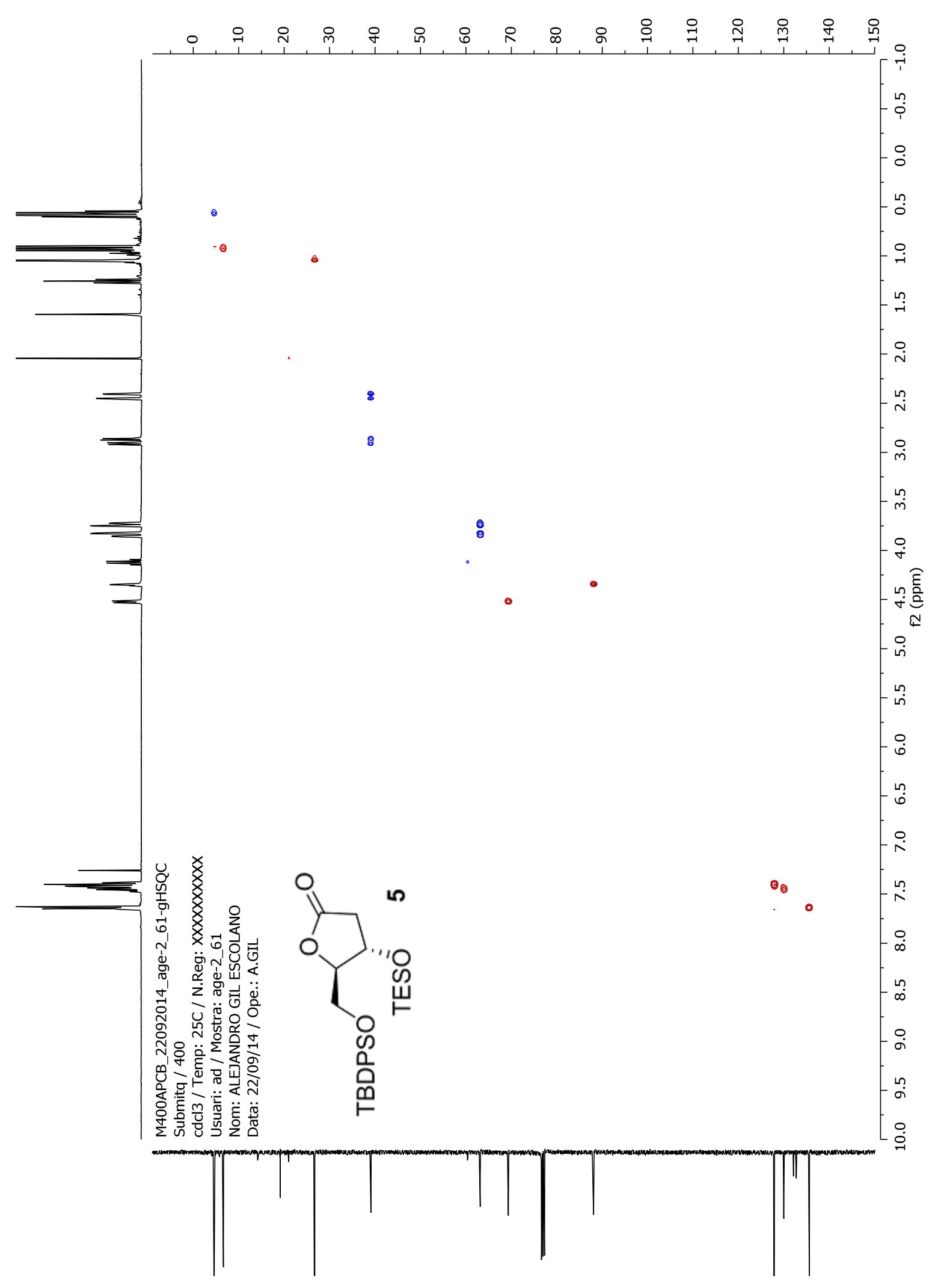




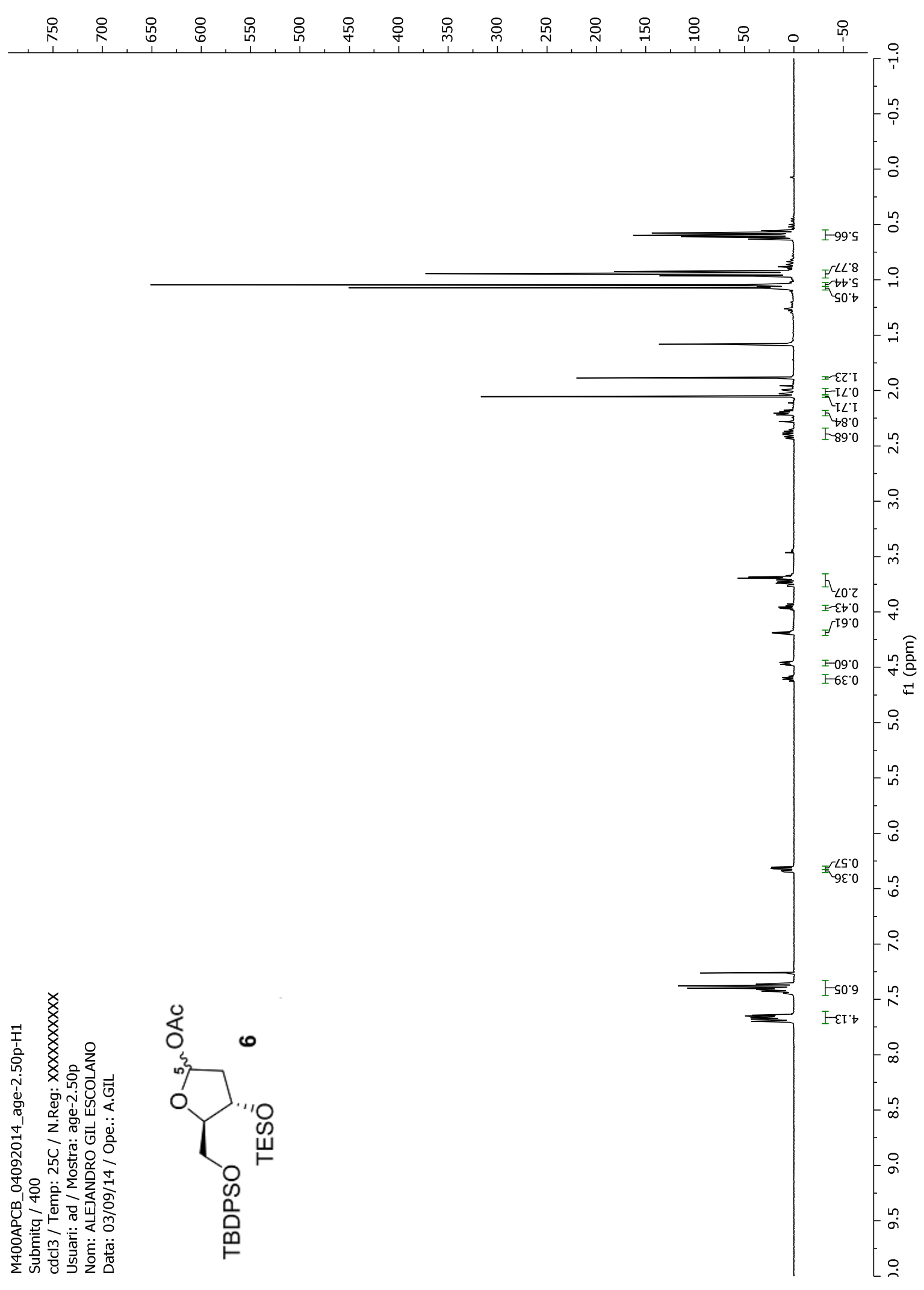




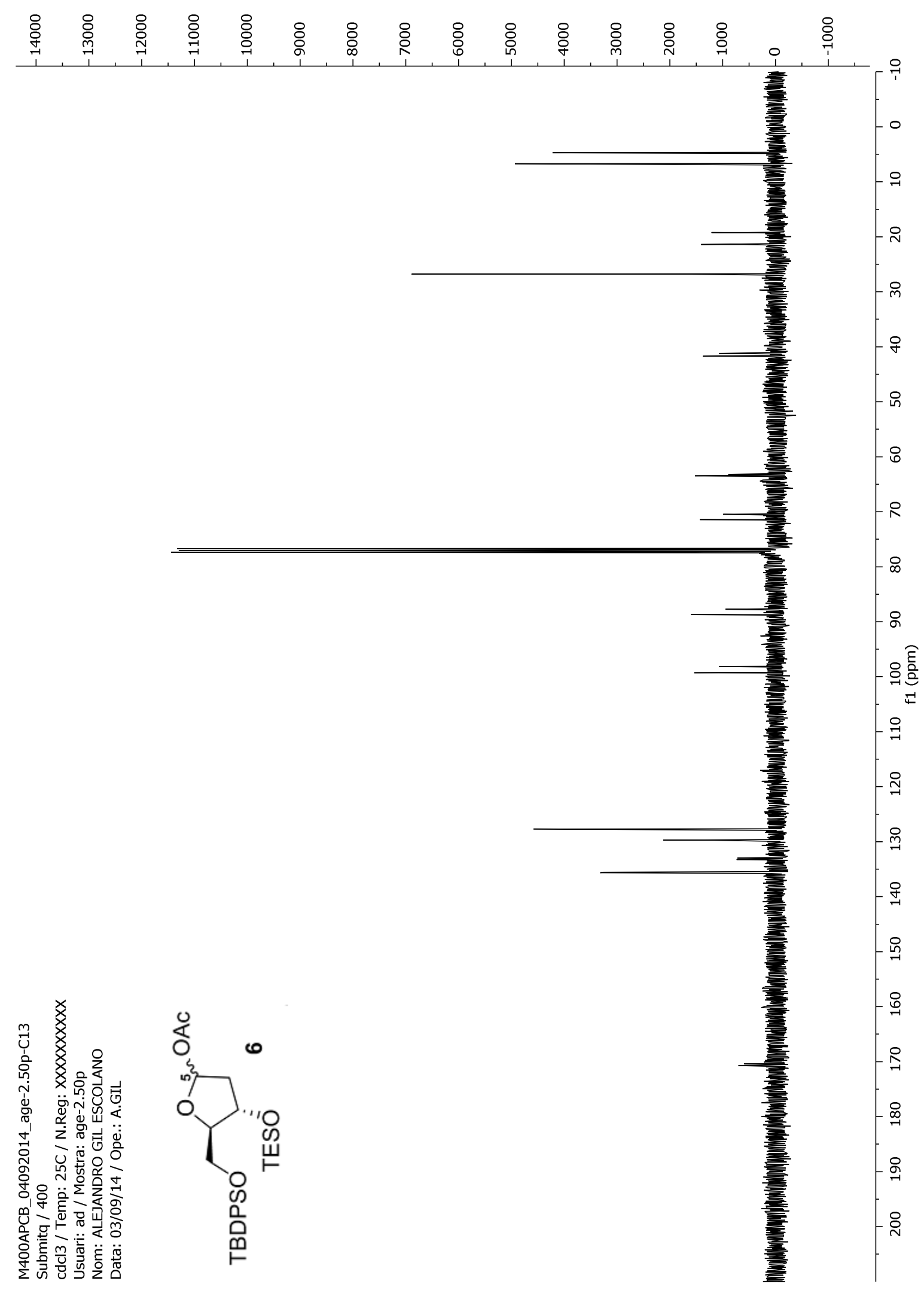


(uidd) If

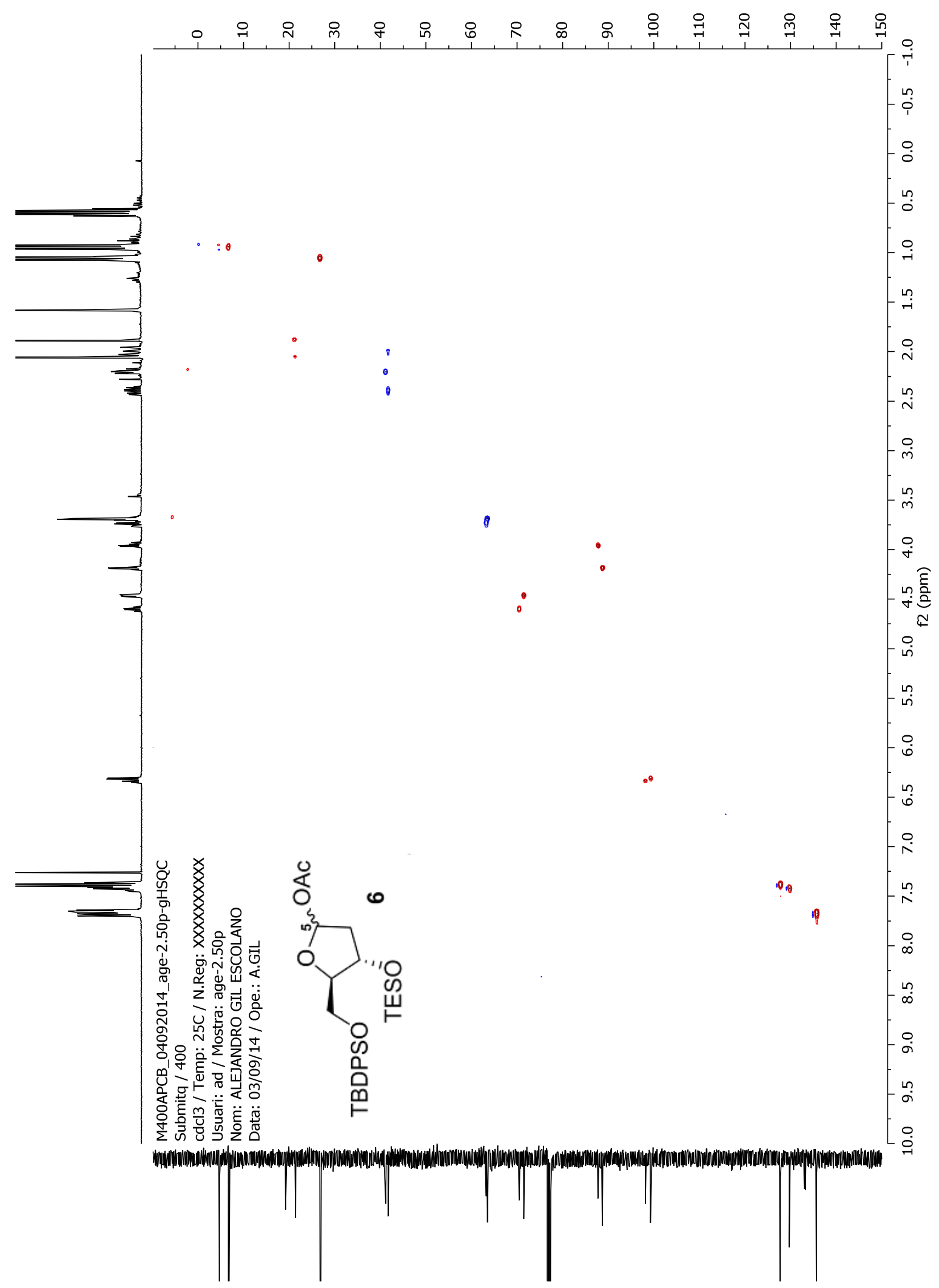




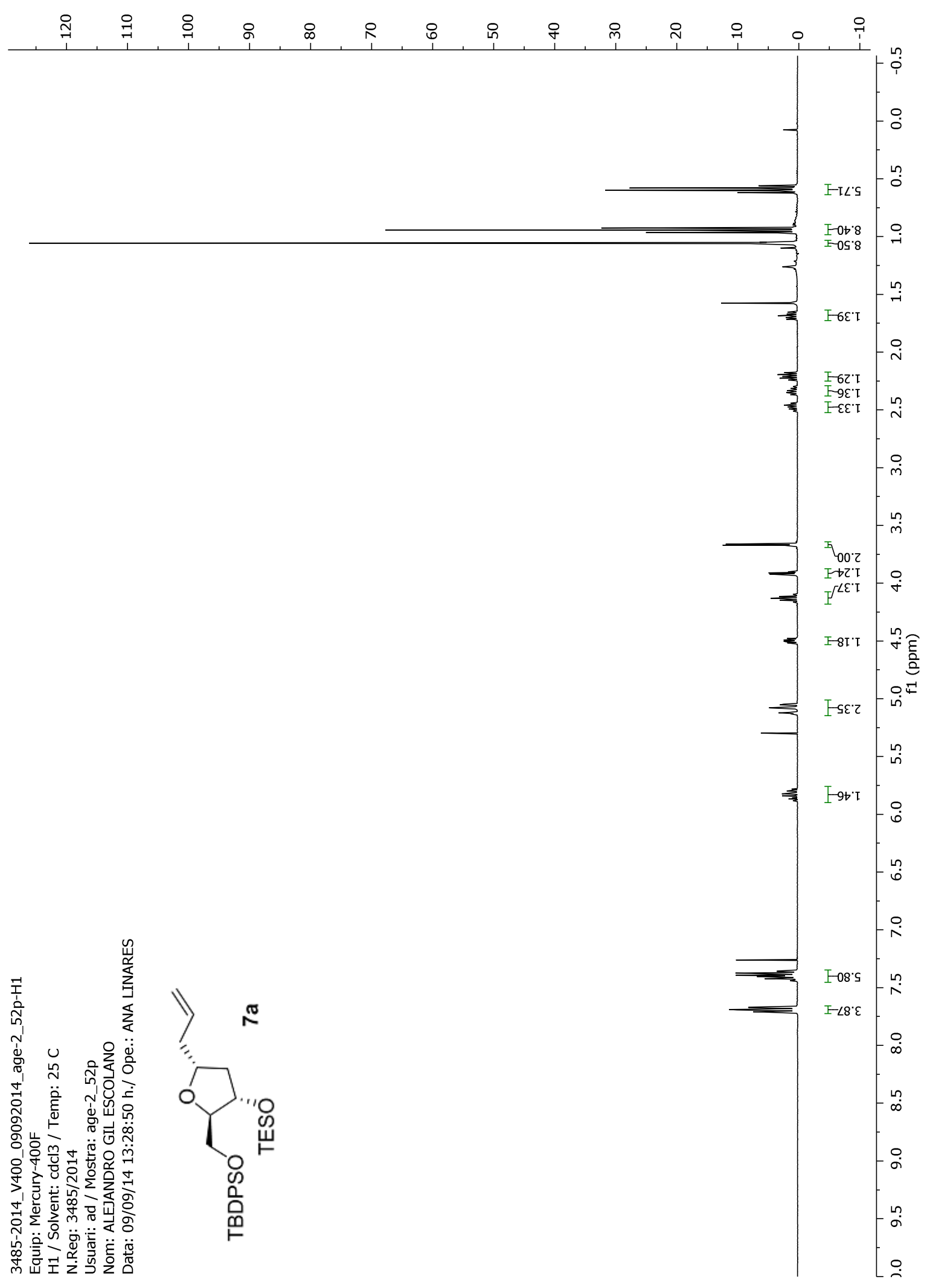




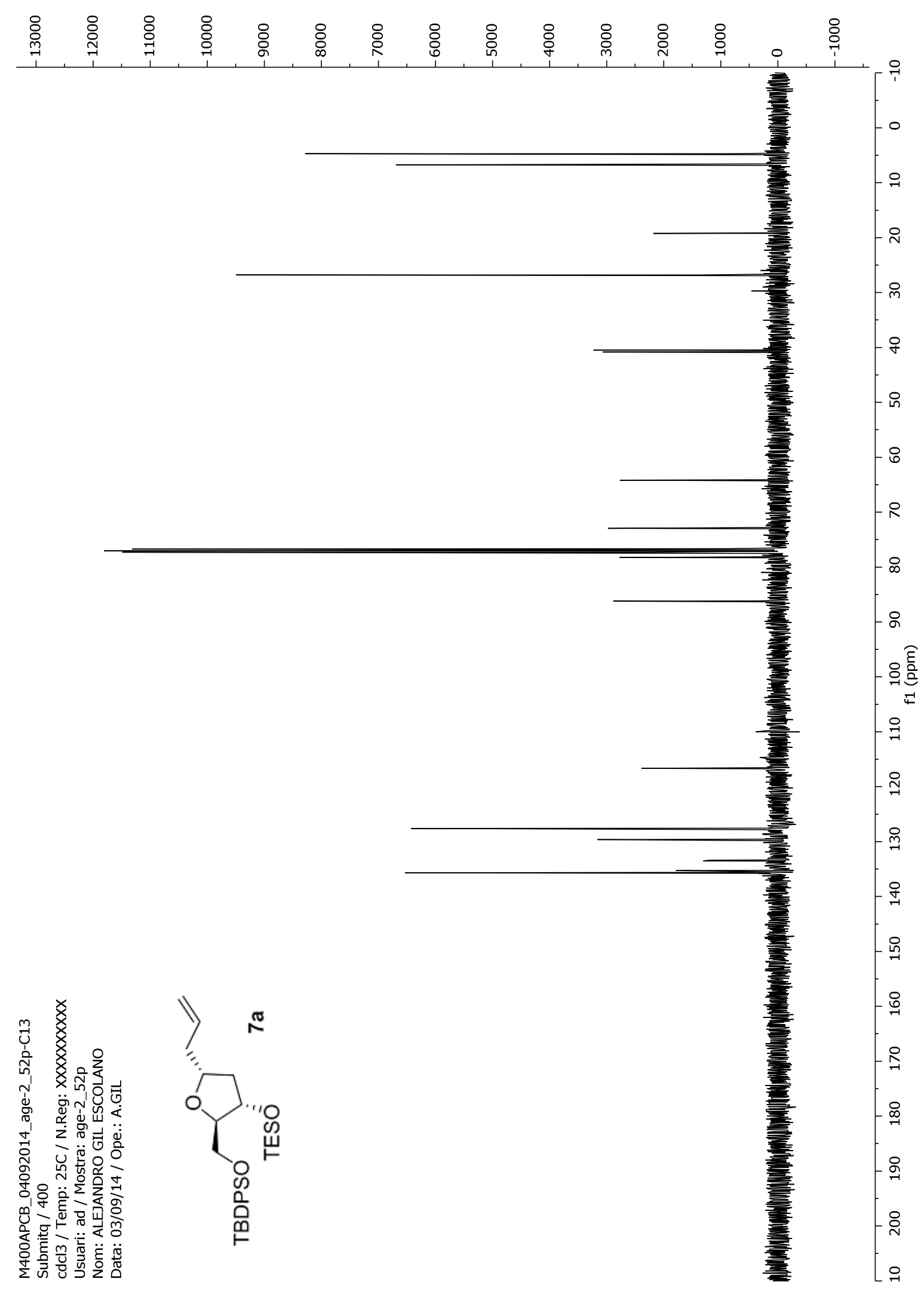


(uidd) If

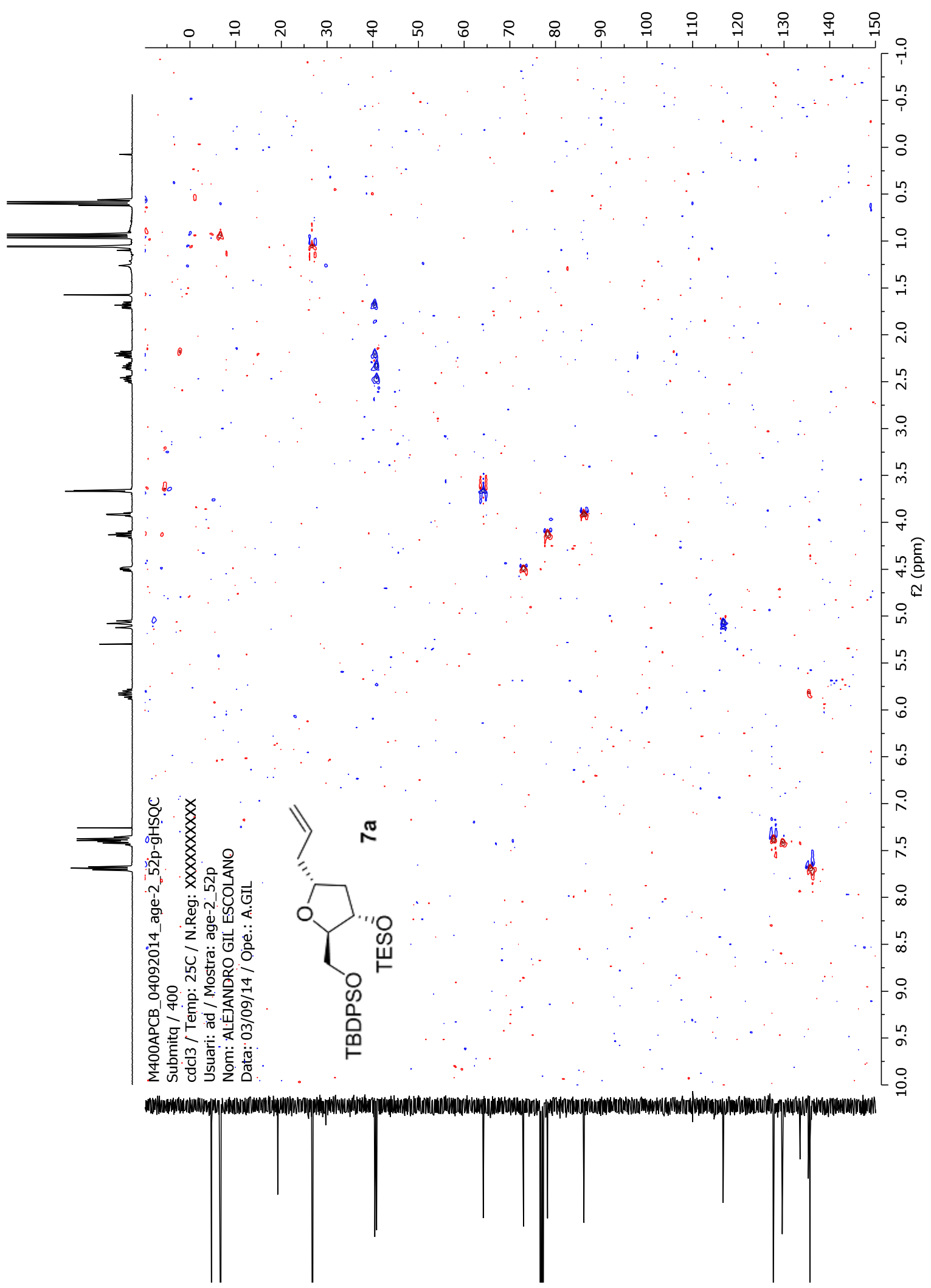




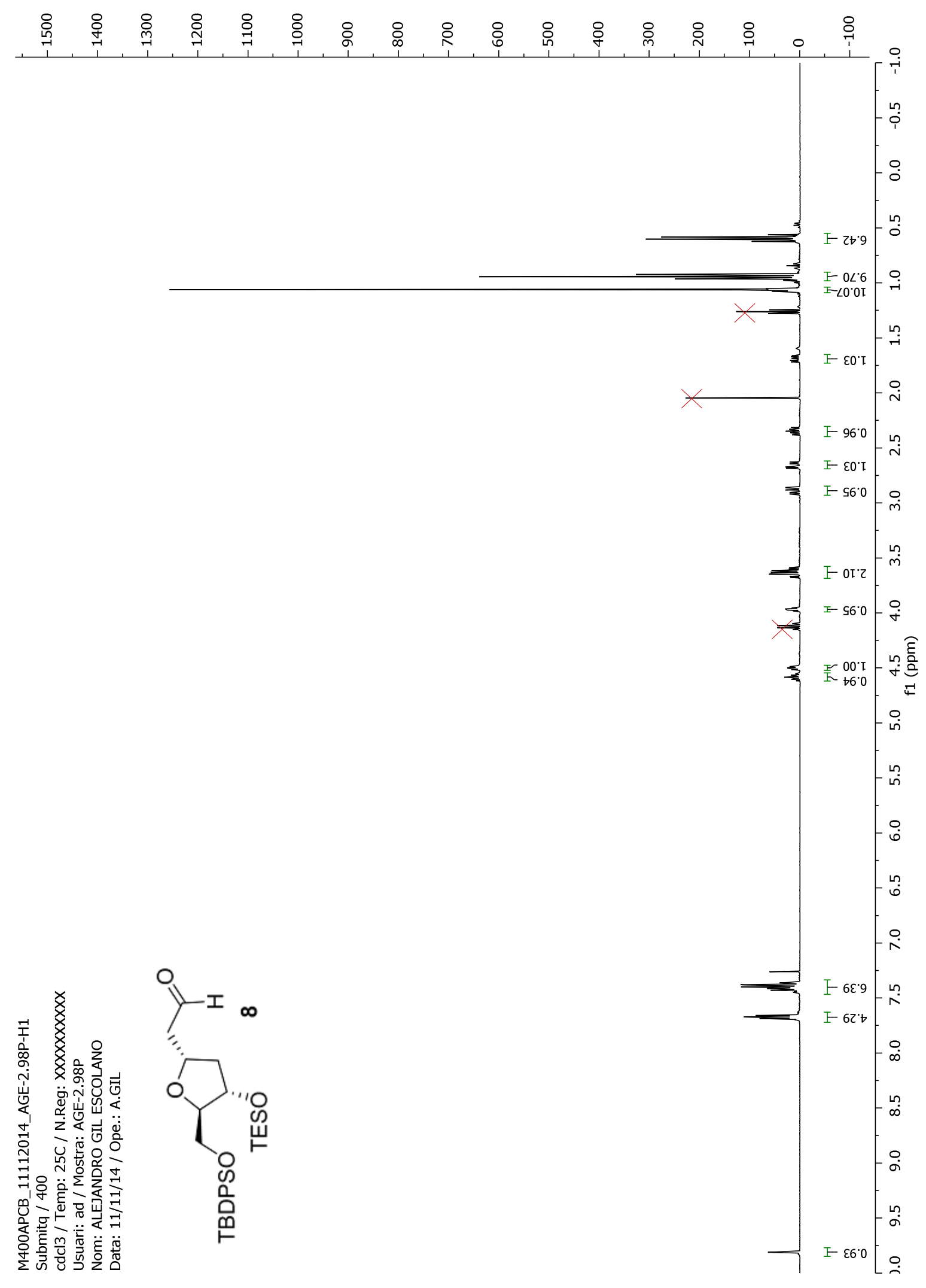




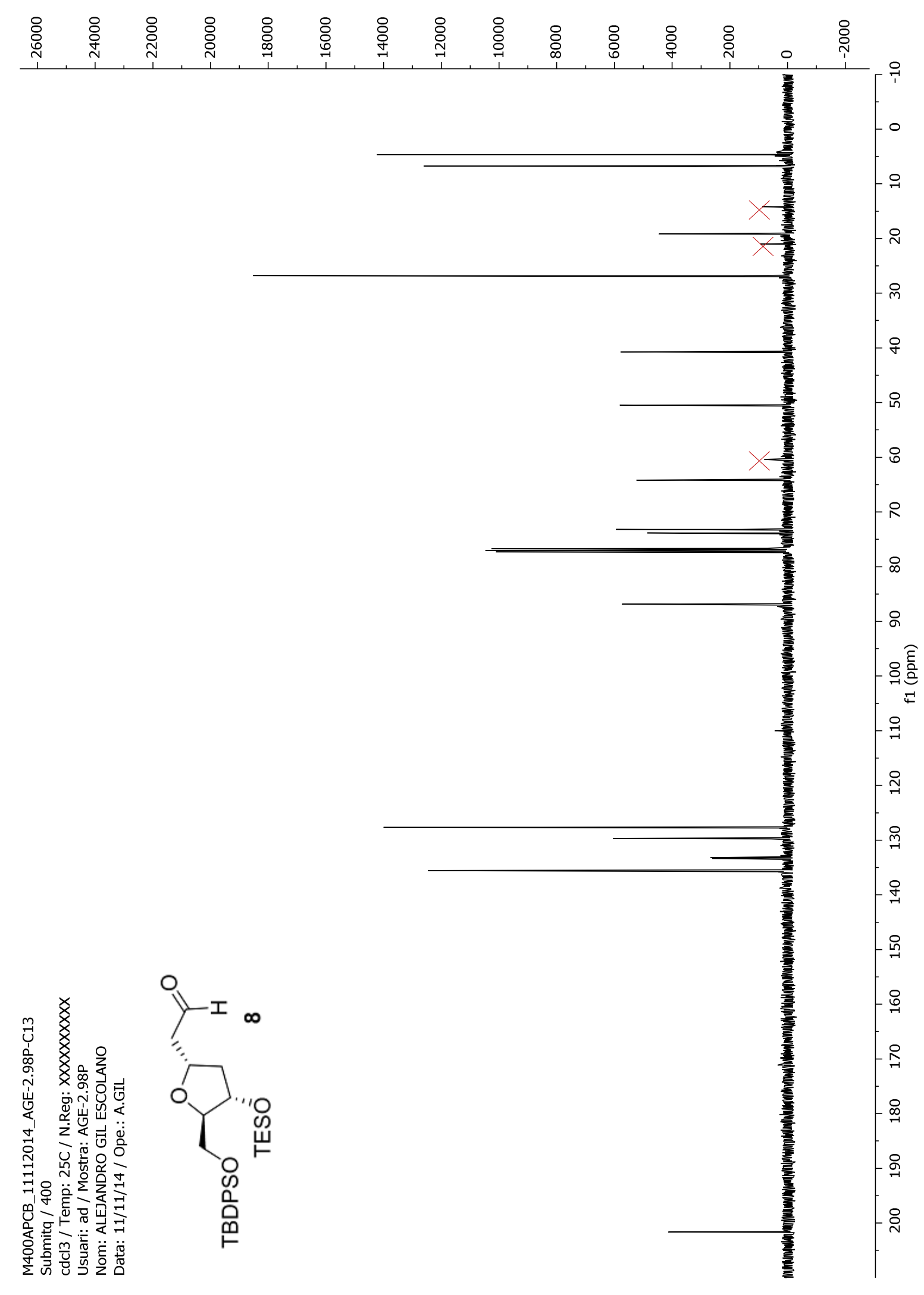


(uidd) If

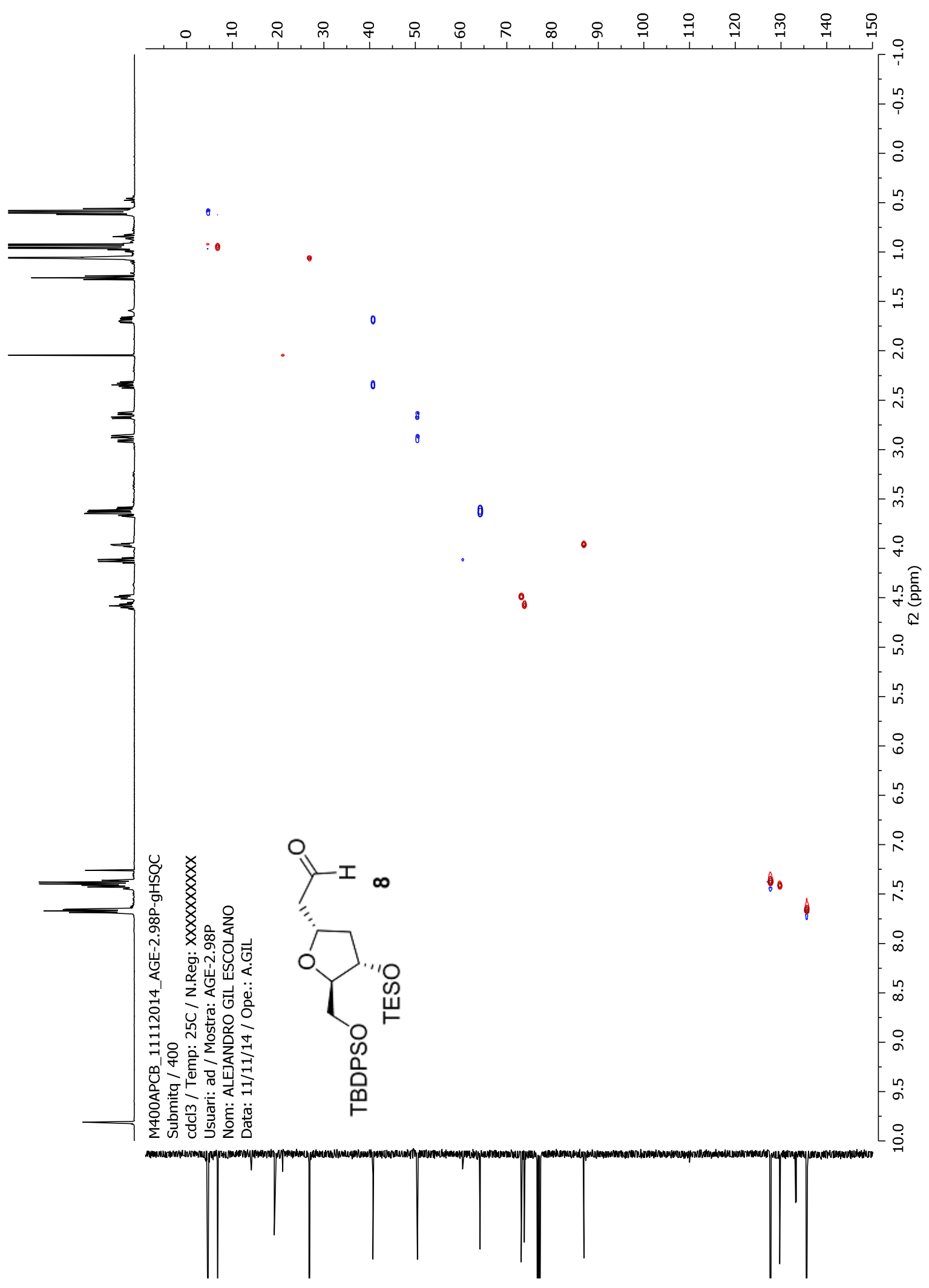




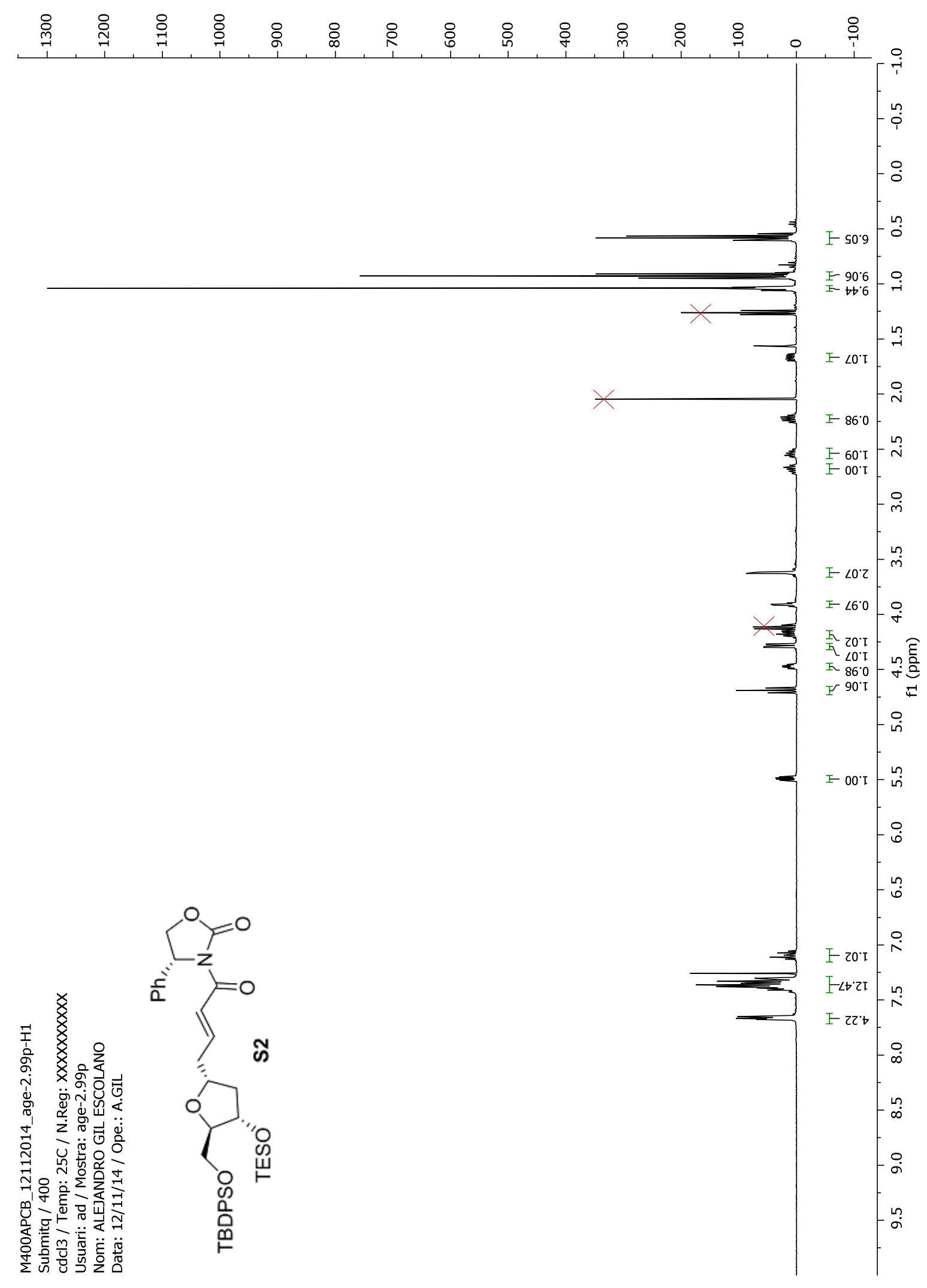




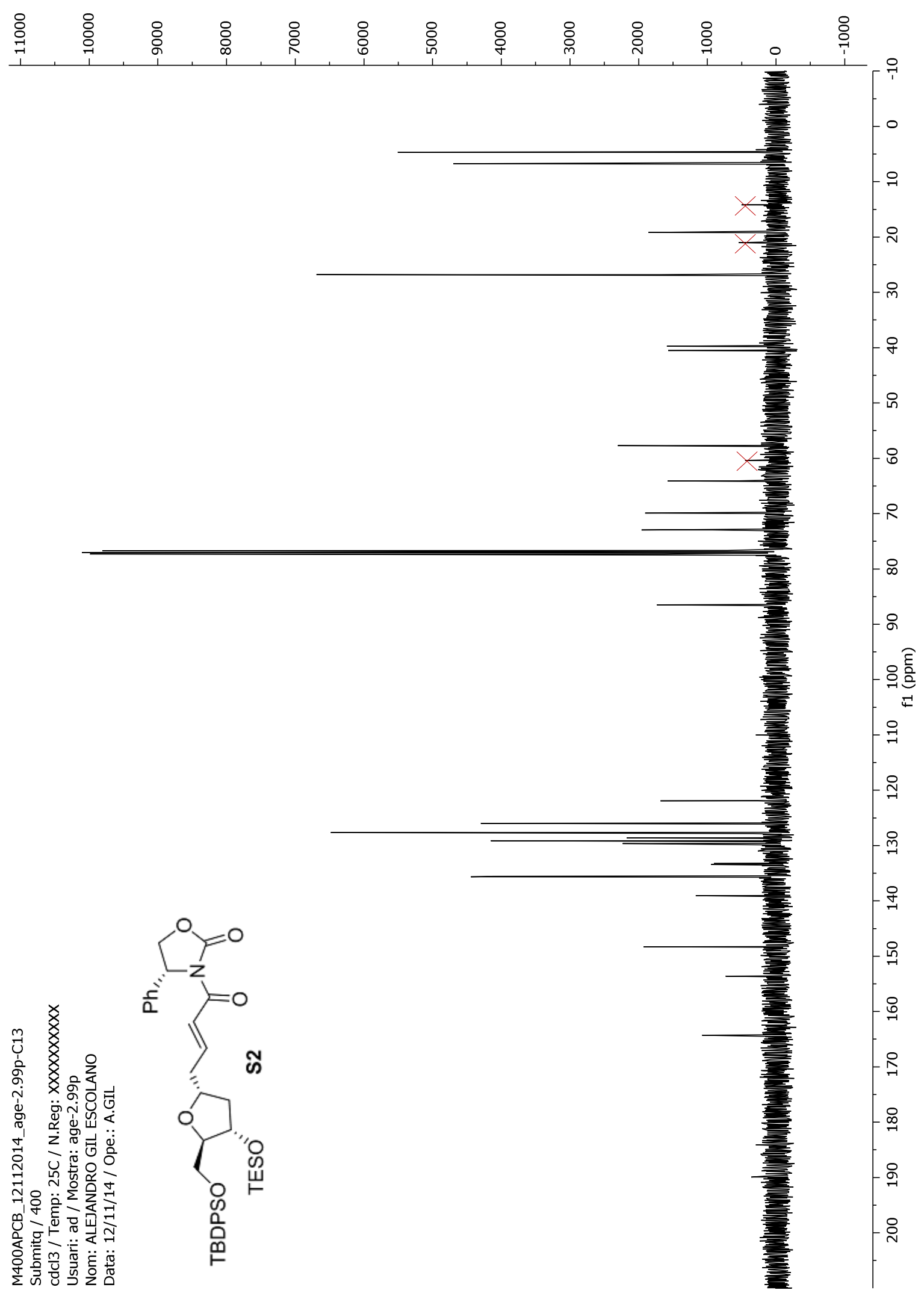


(udd) If

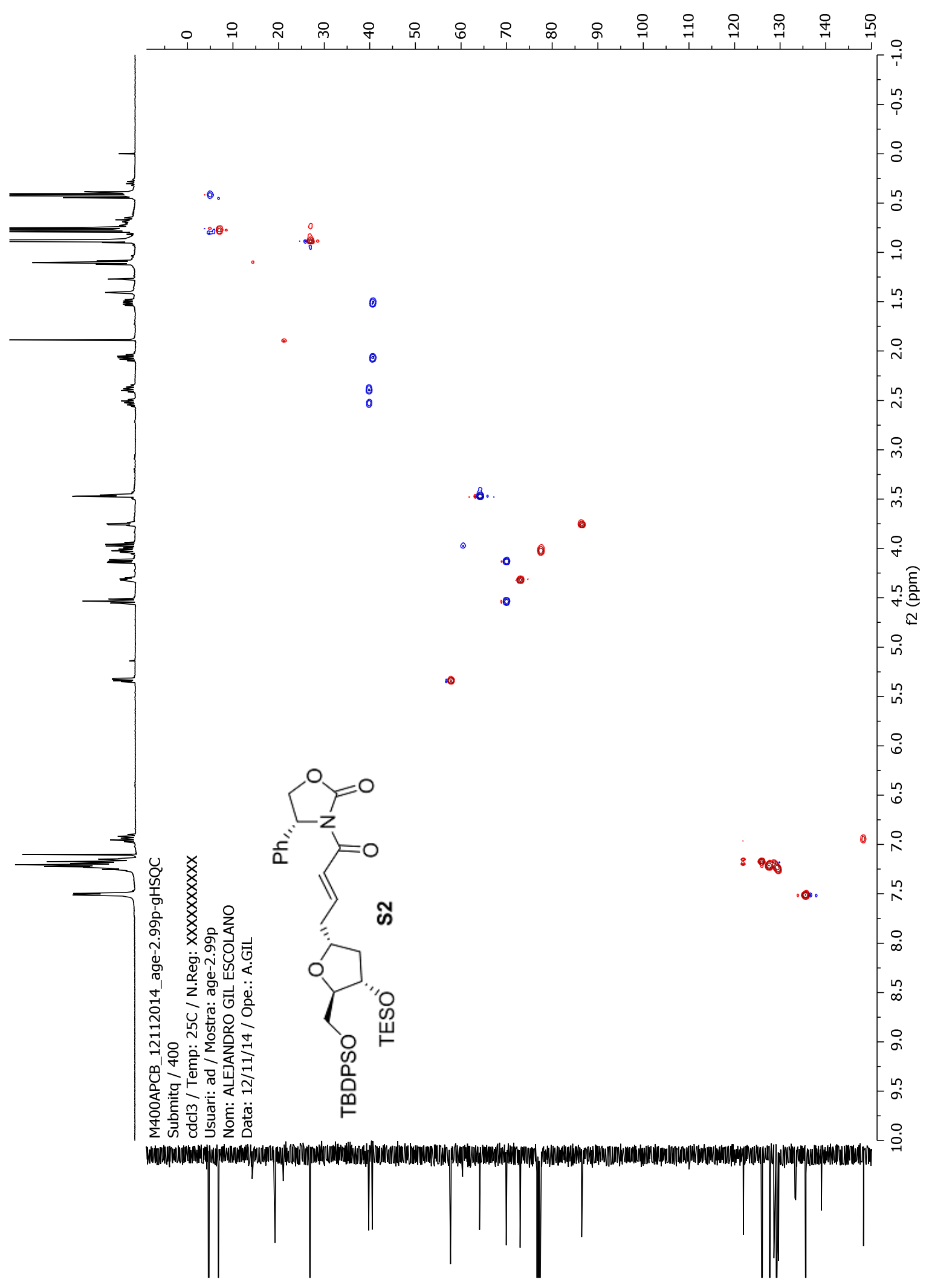




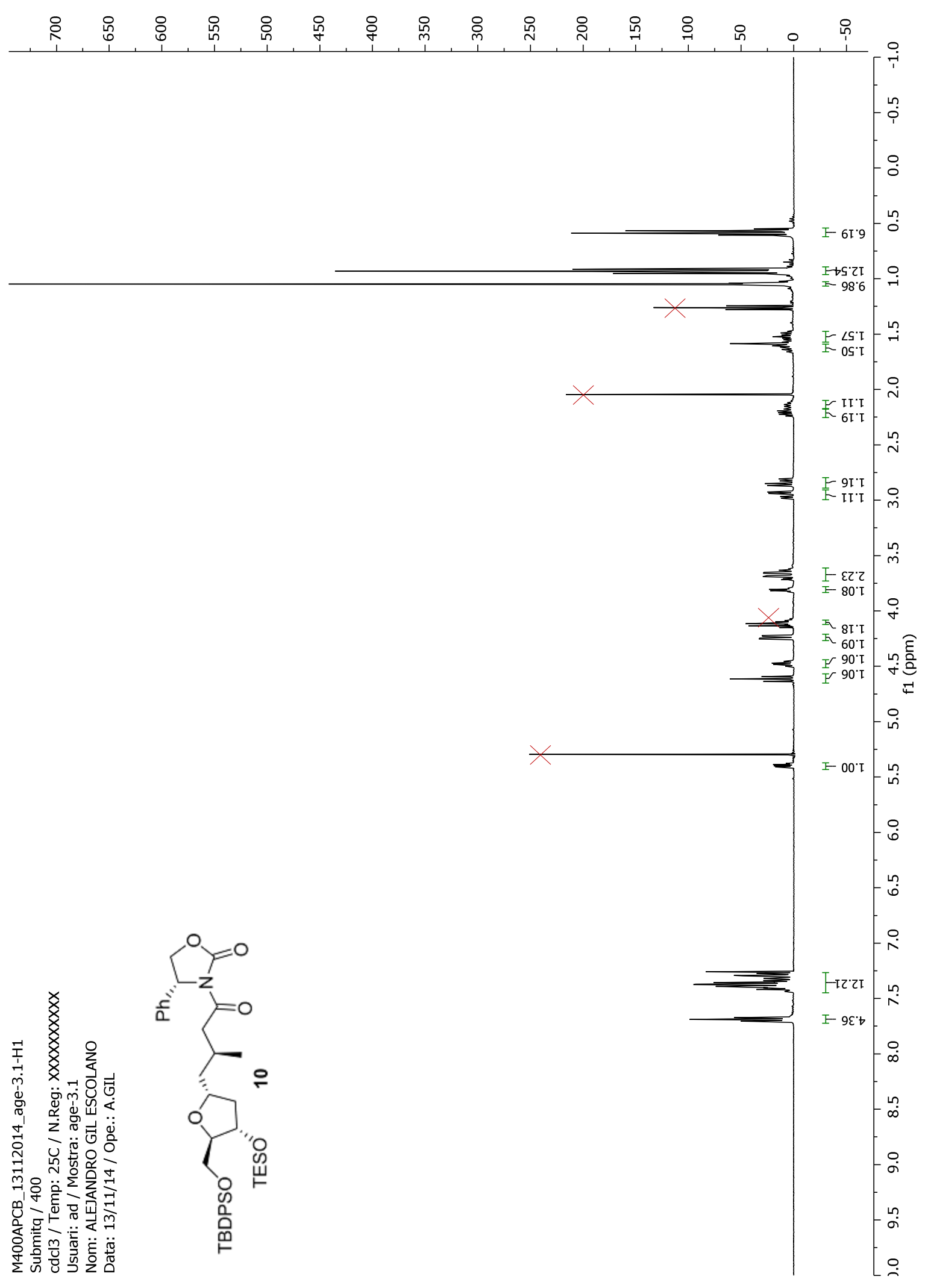




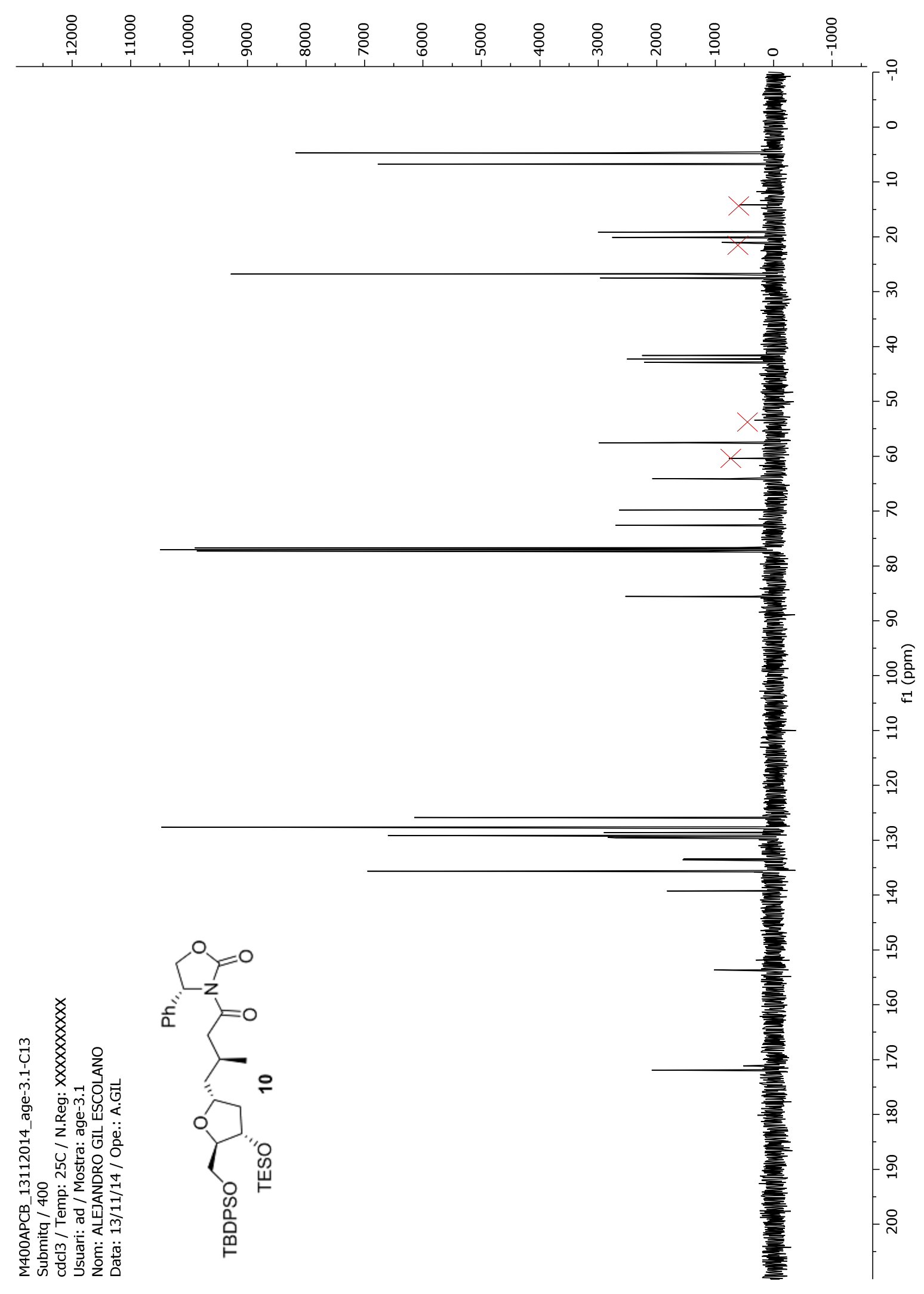


(udd) If

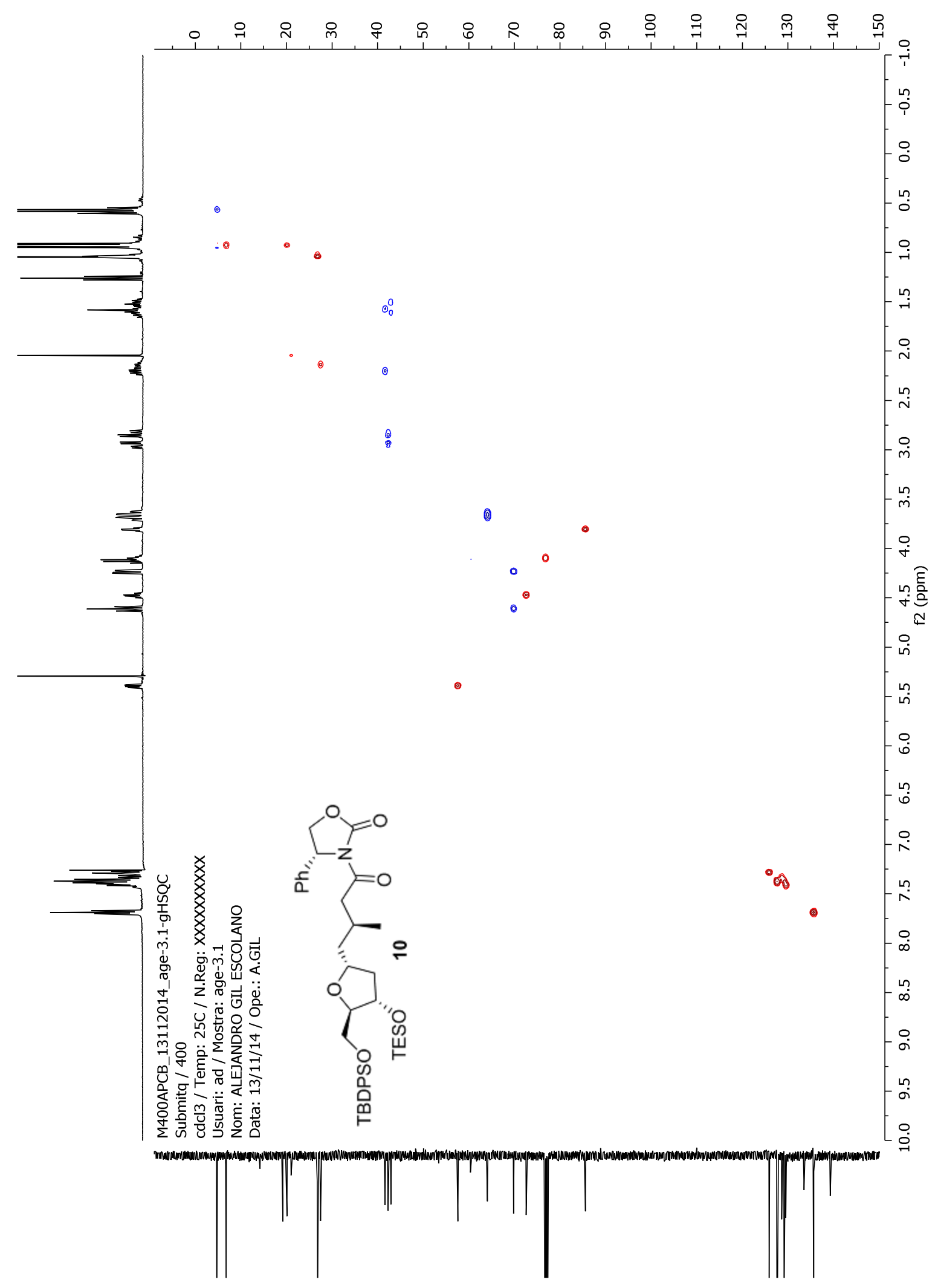




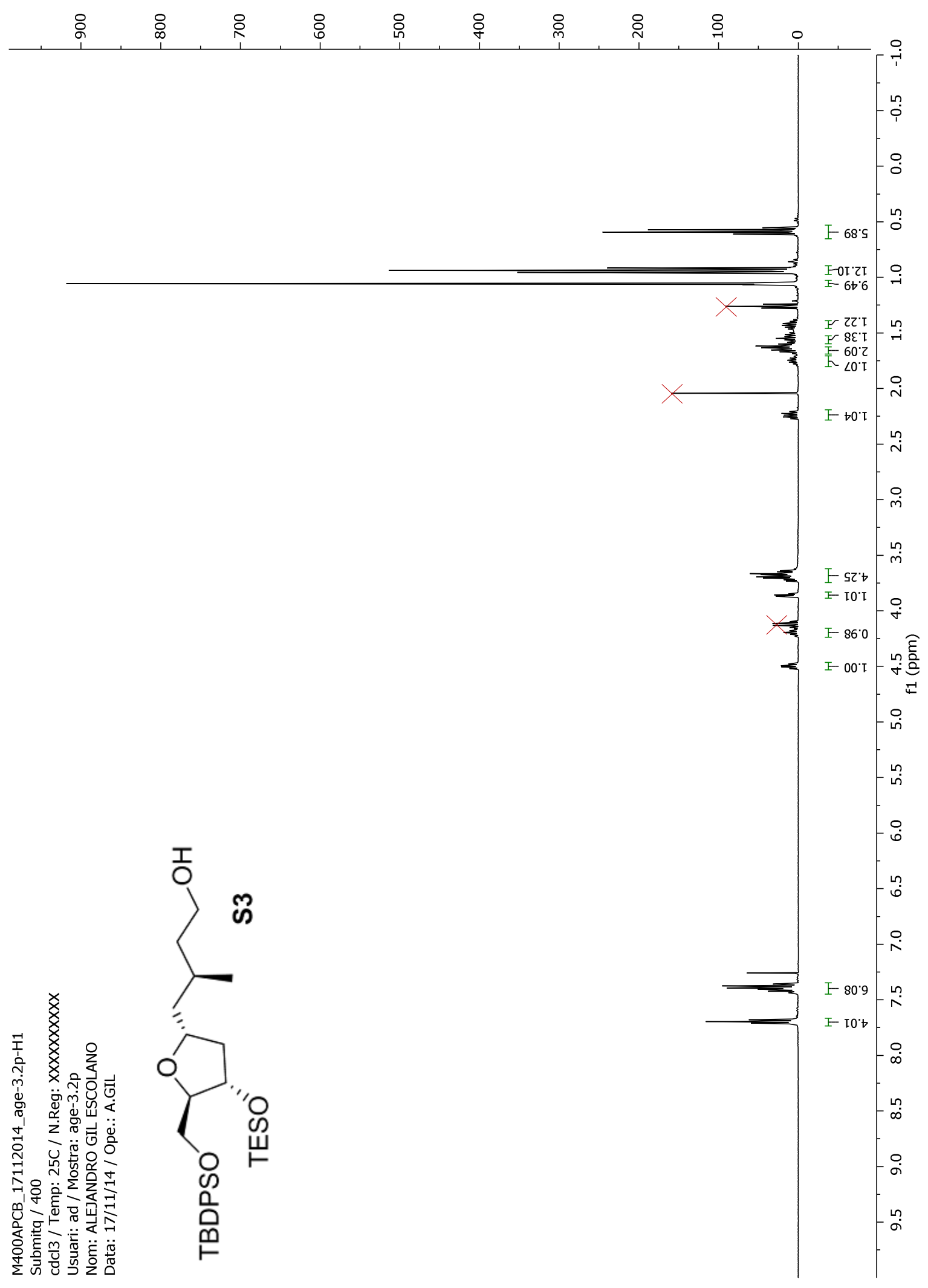




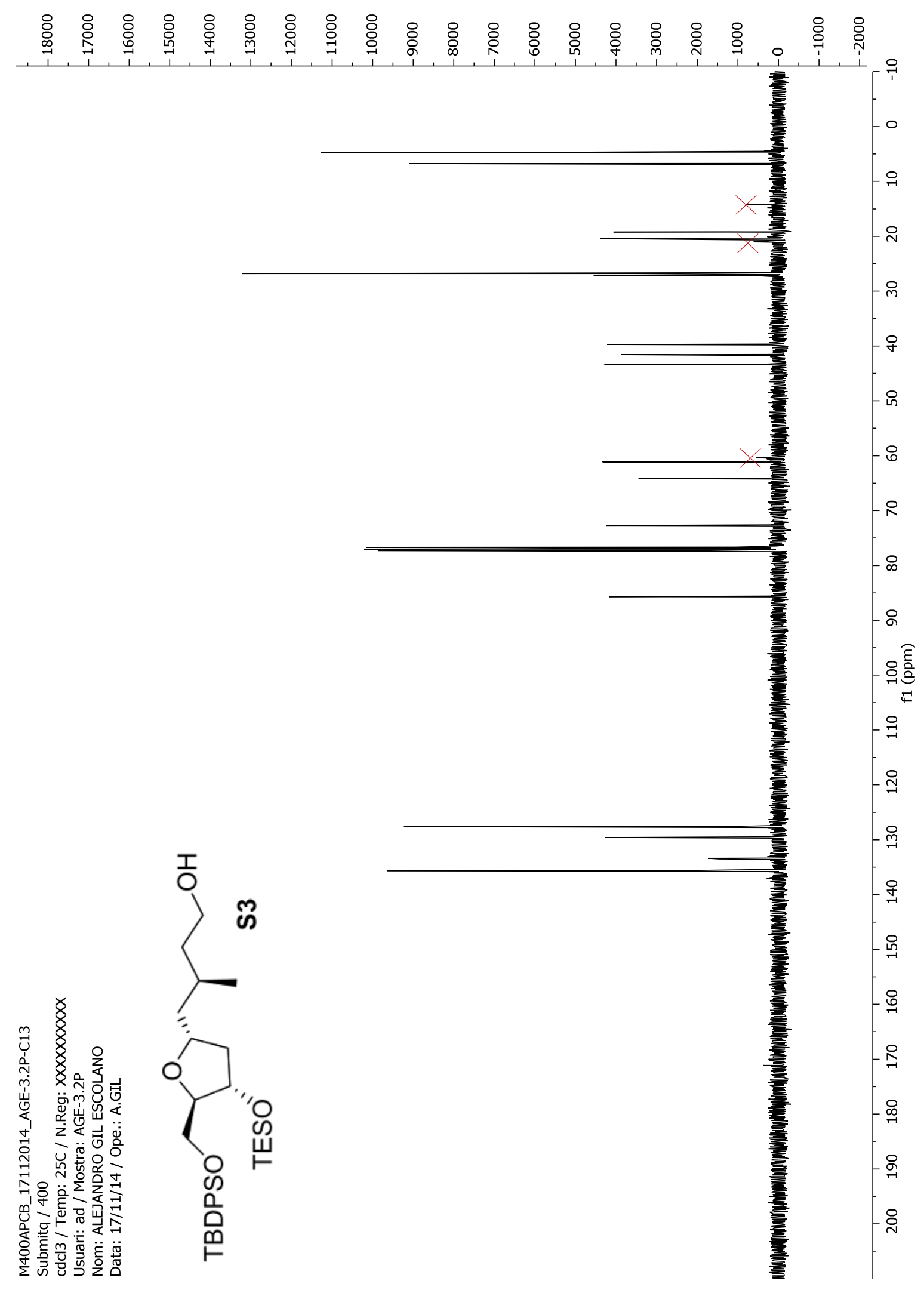


(udd) If

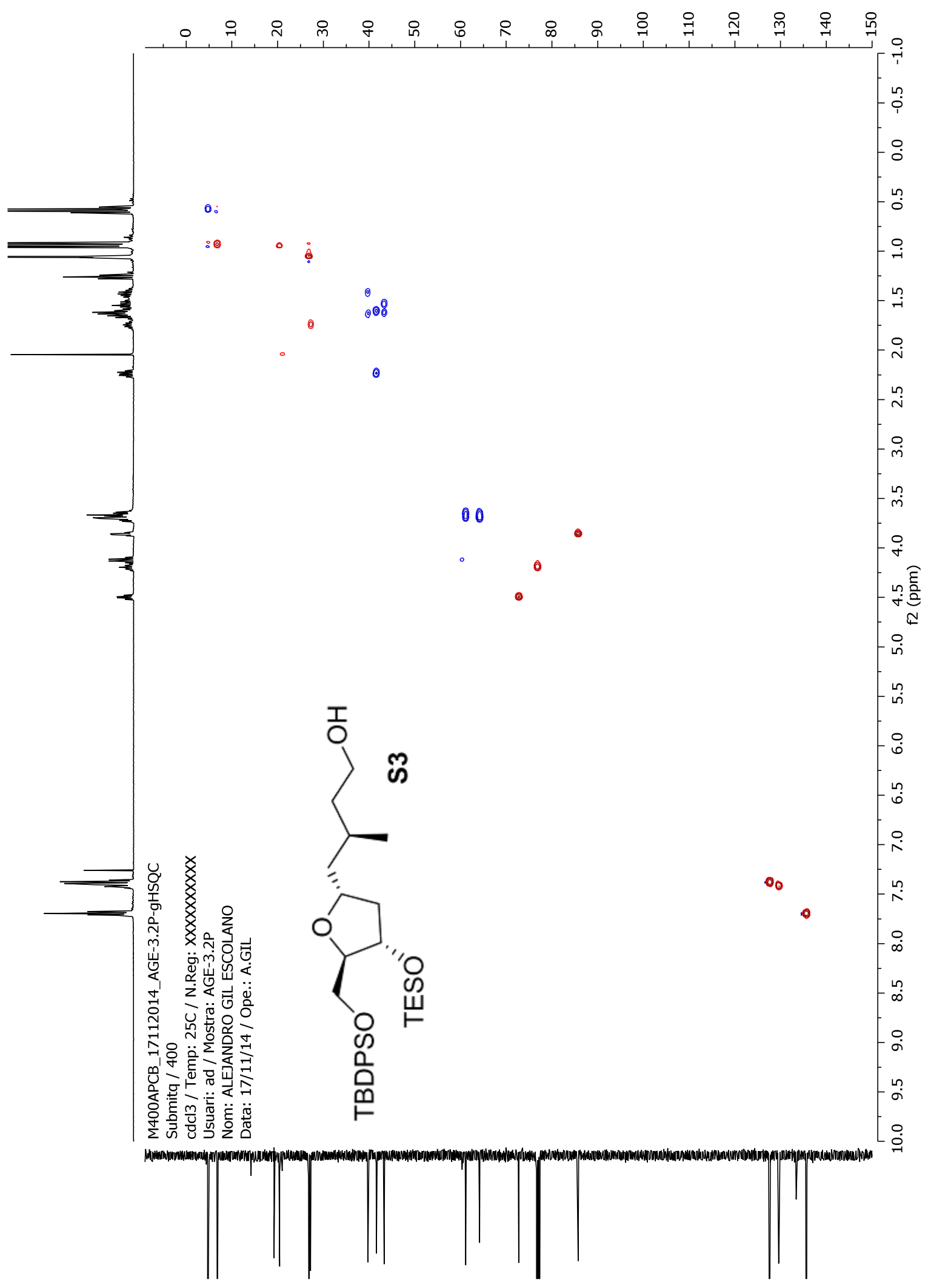




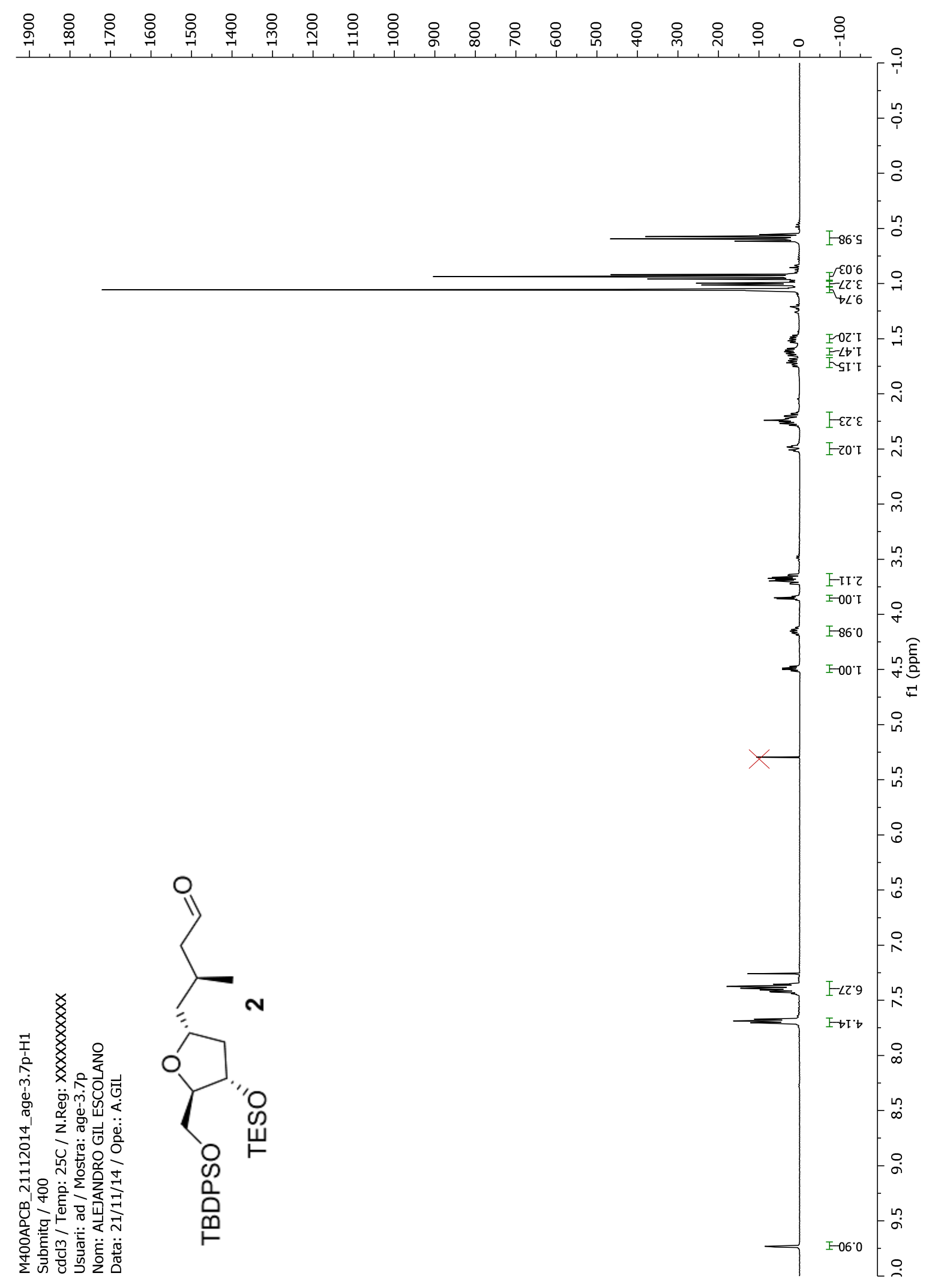




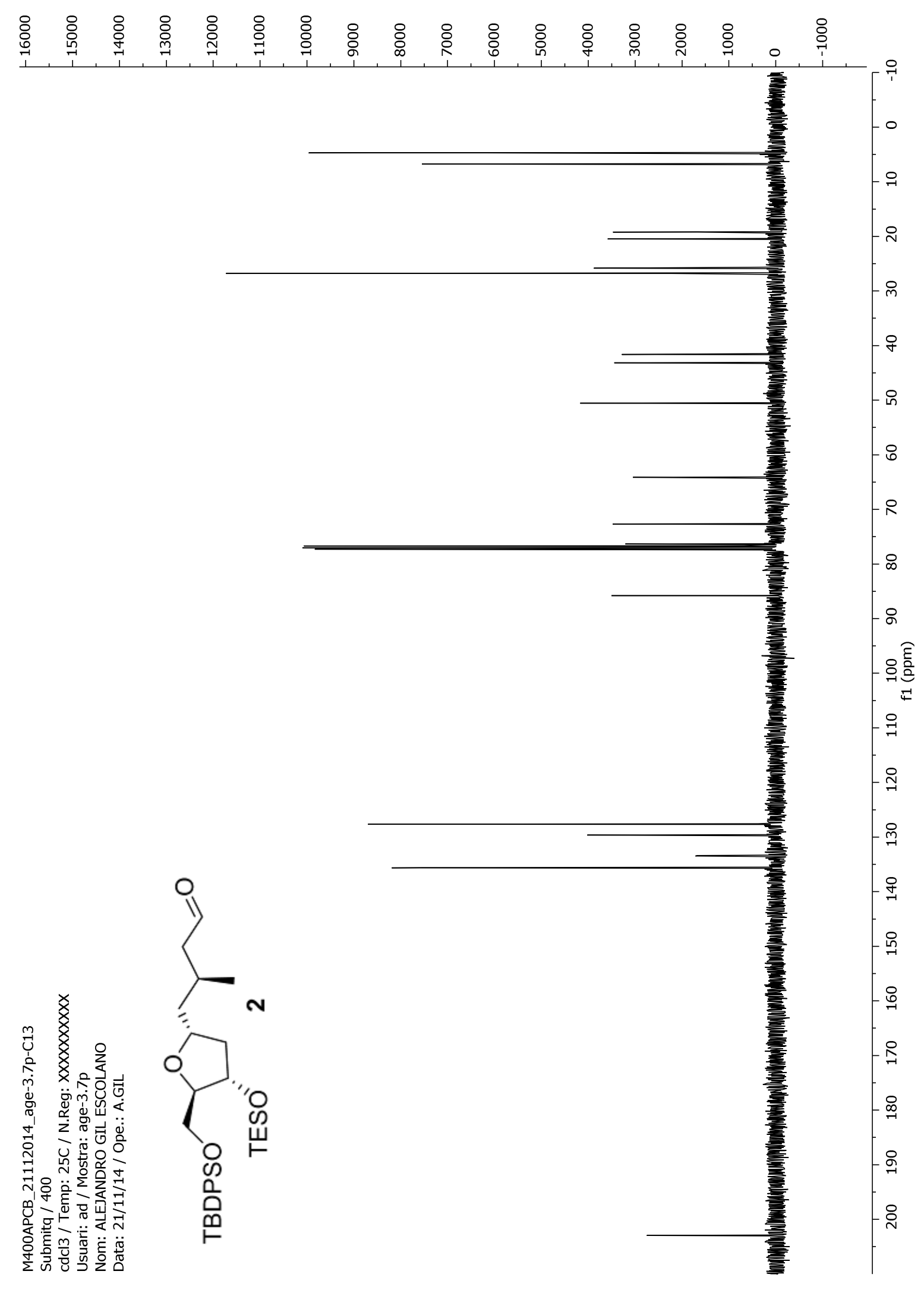


(udd) If

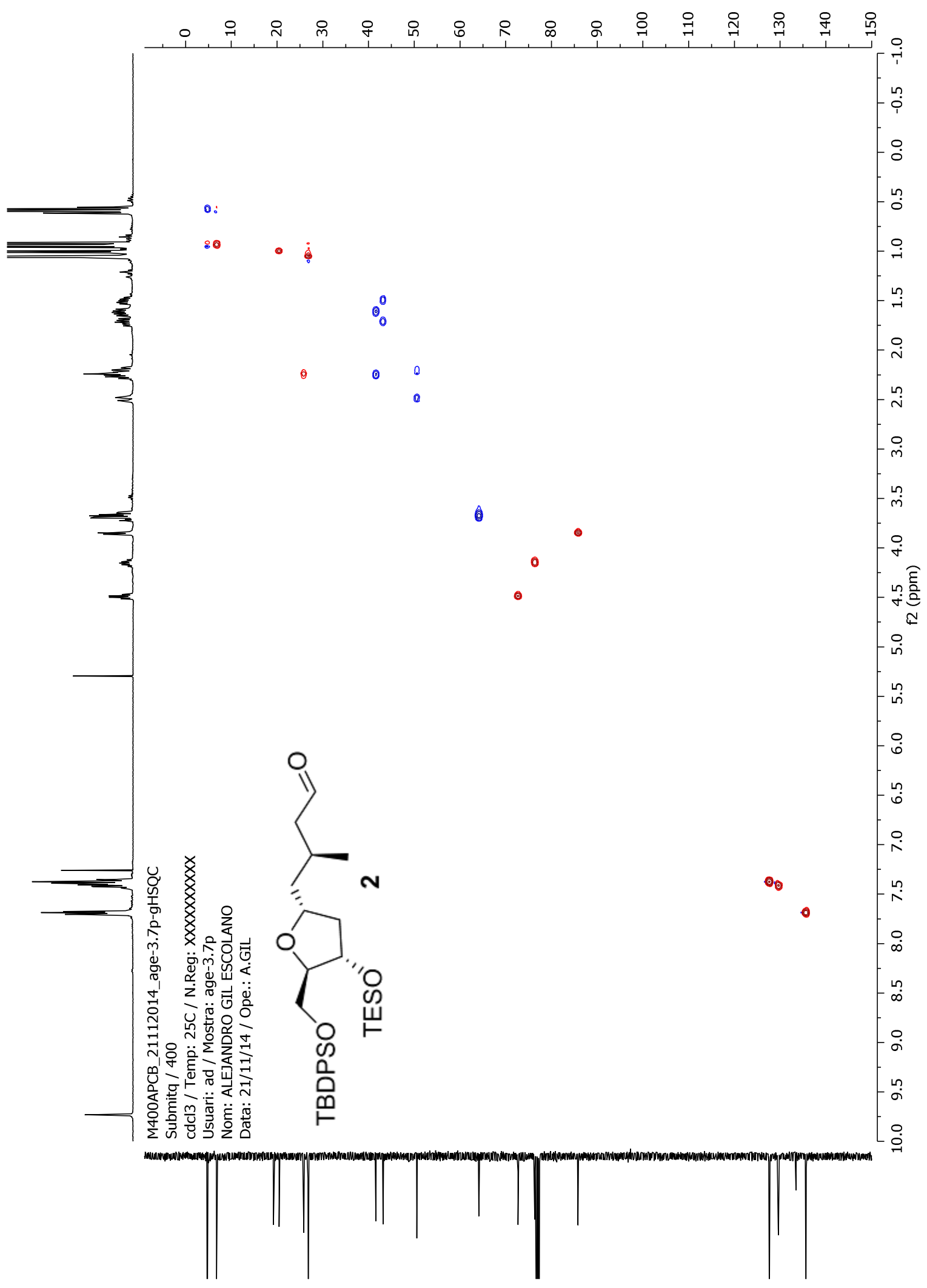




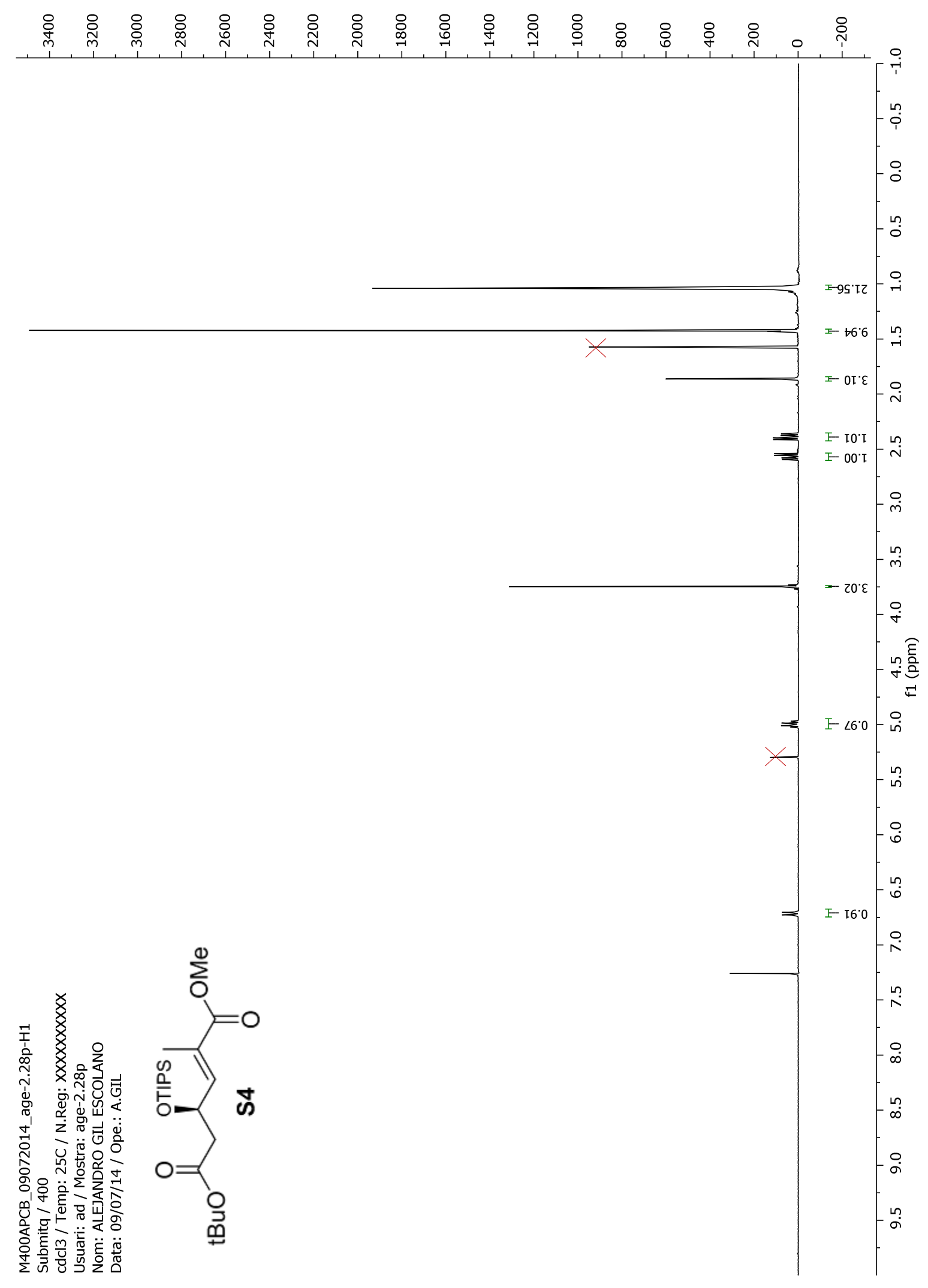




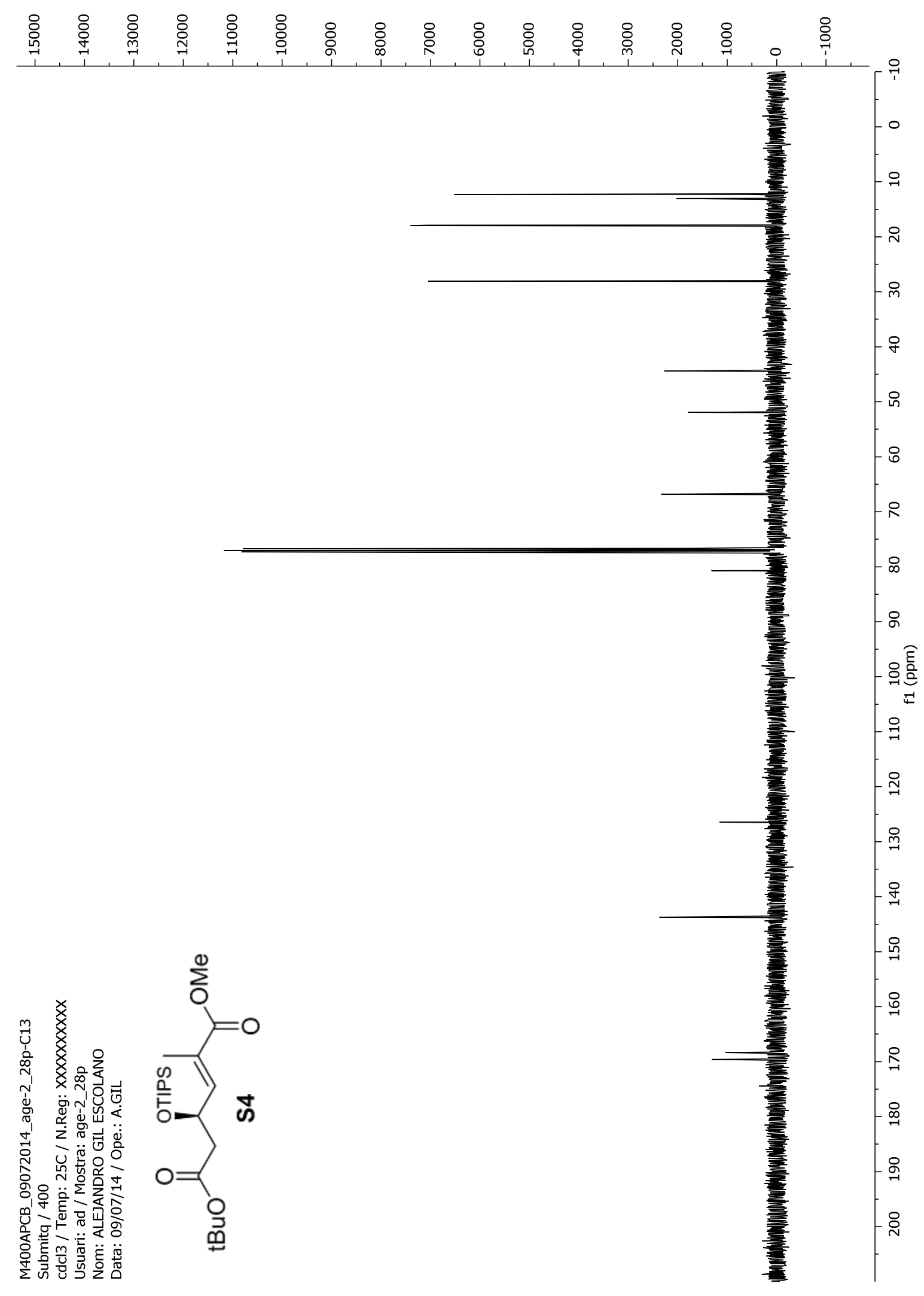


(udd) If

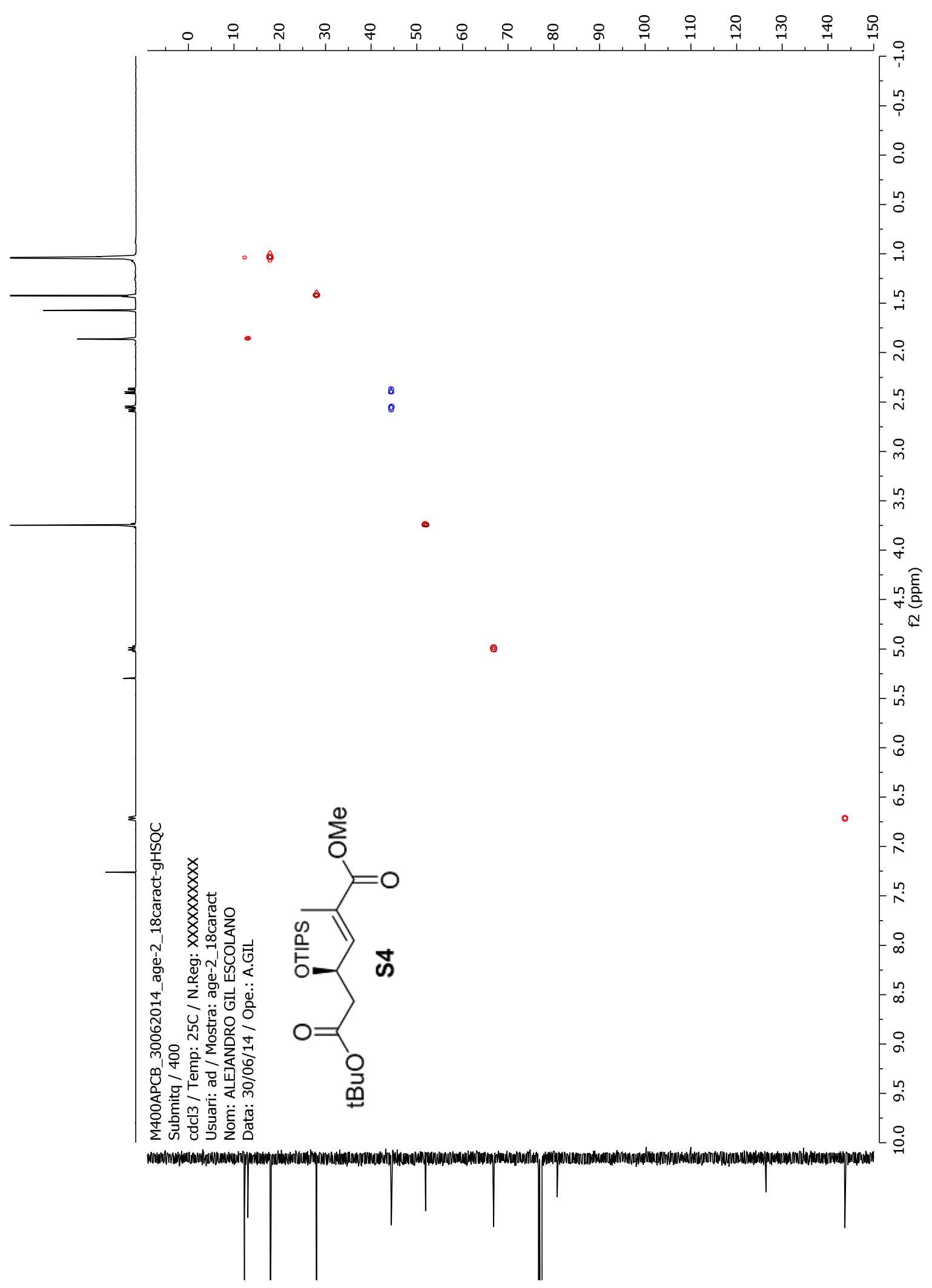




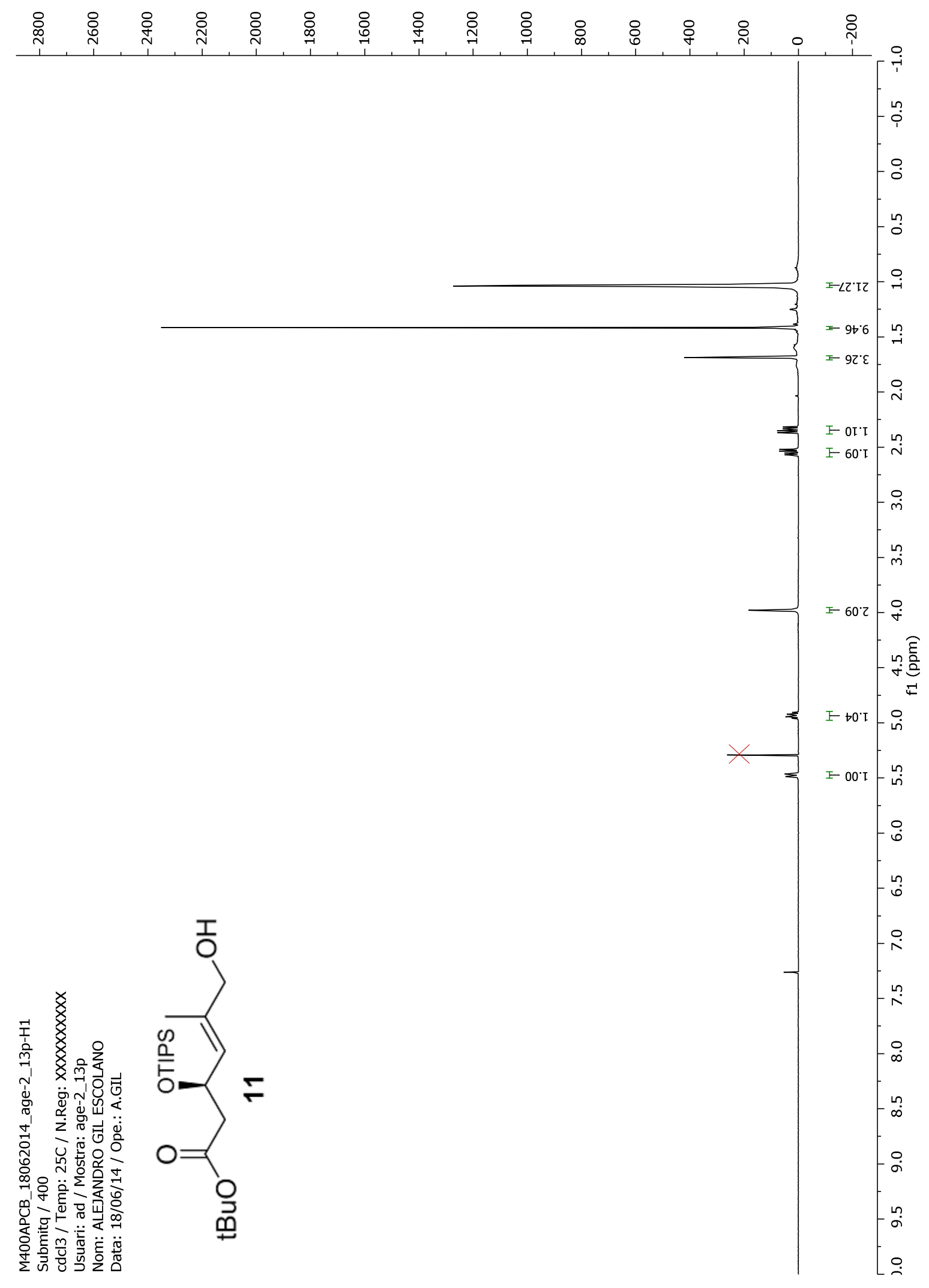




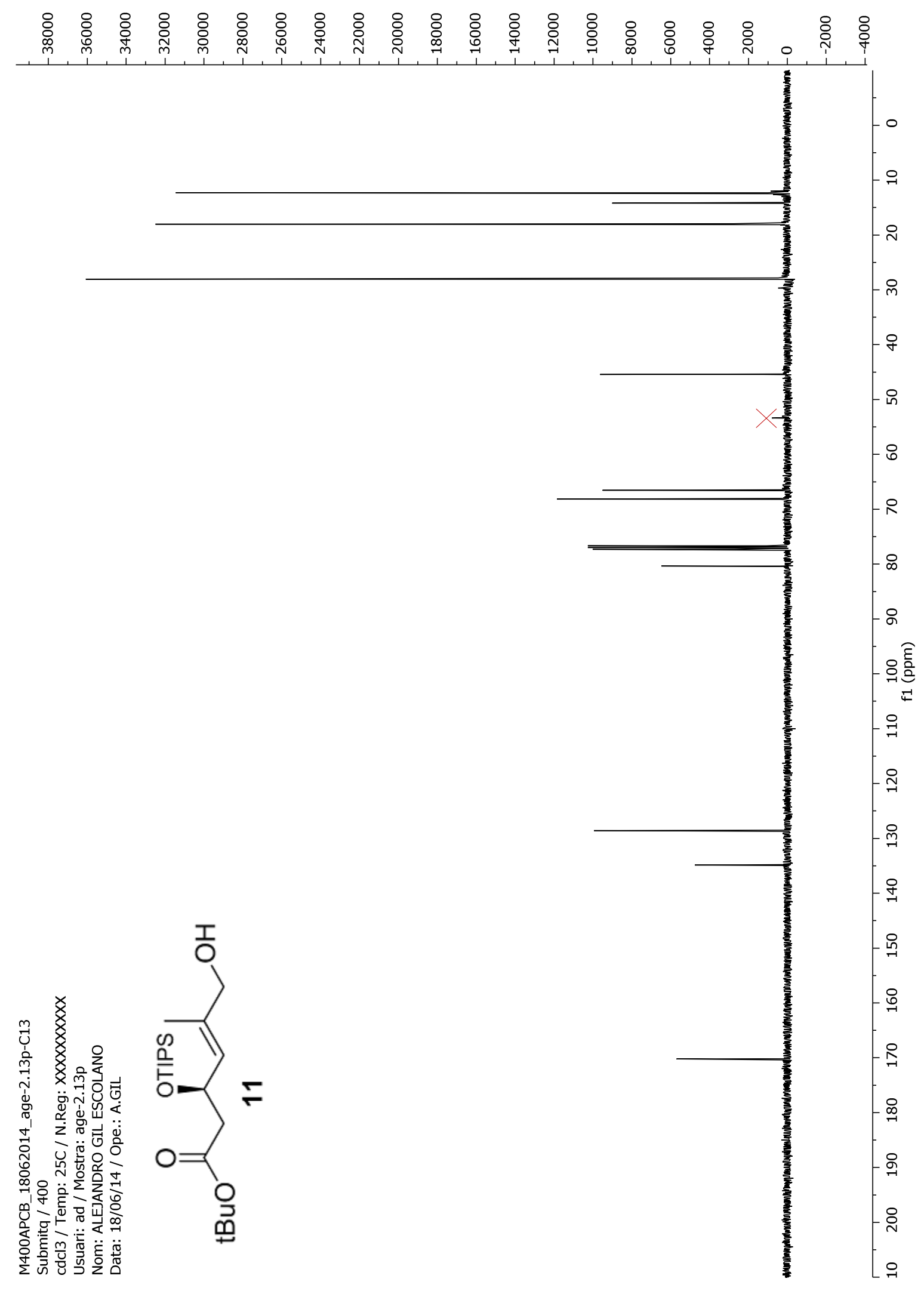


(udd) if

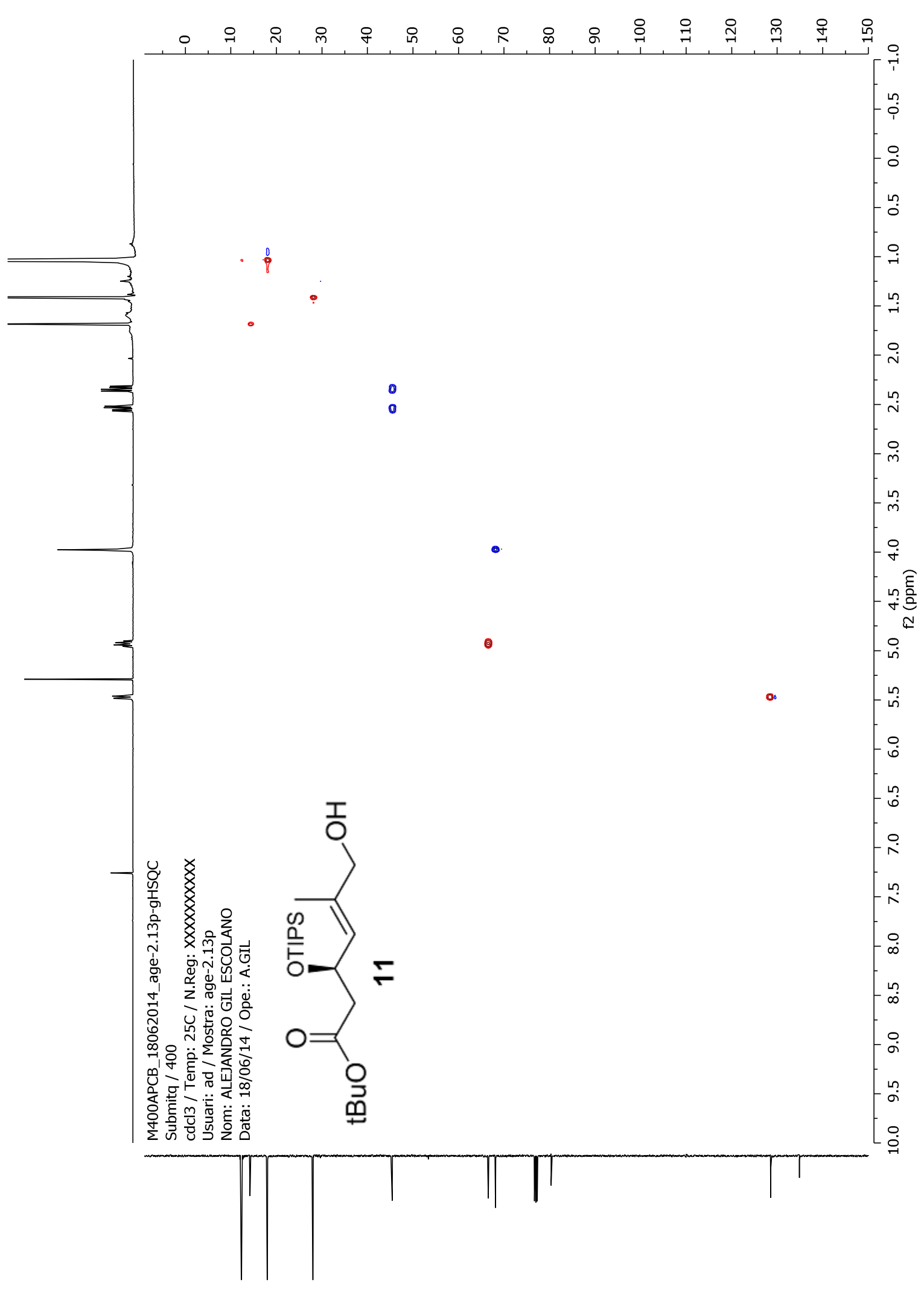




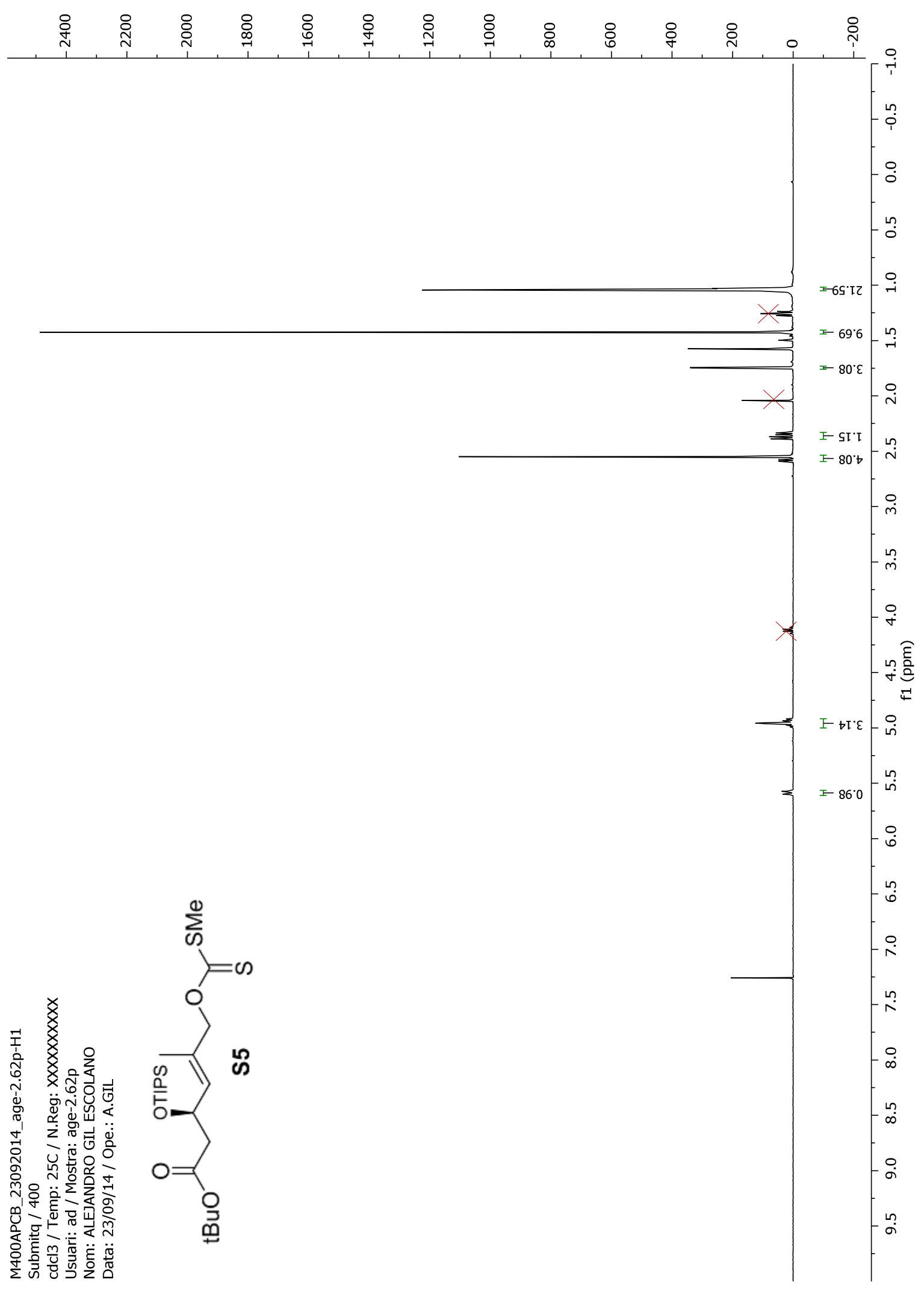




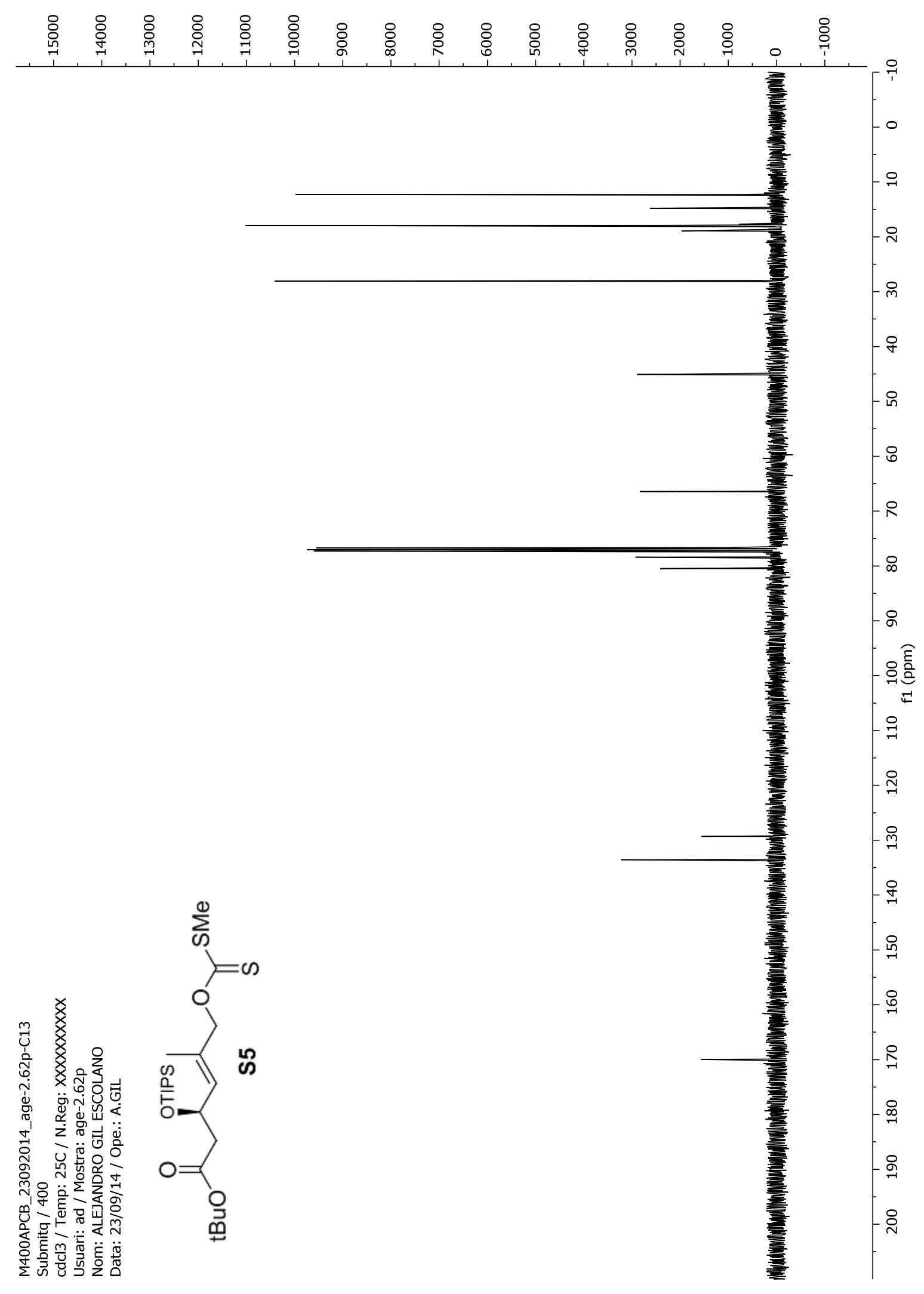


(udd) If

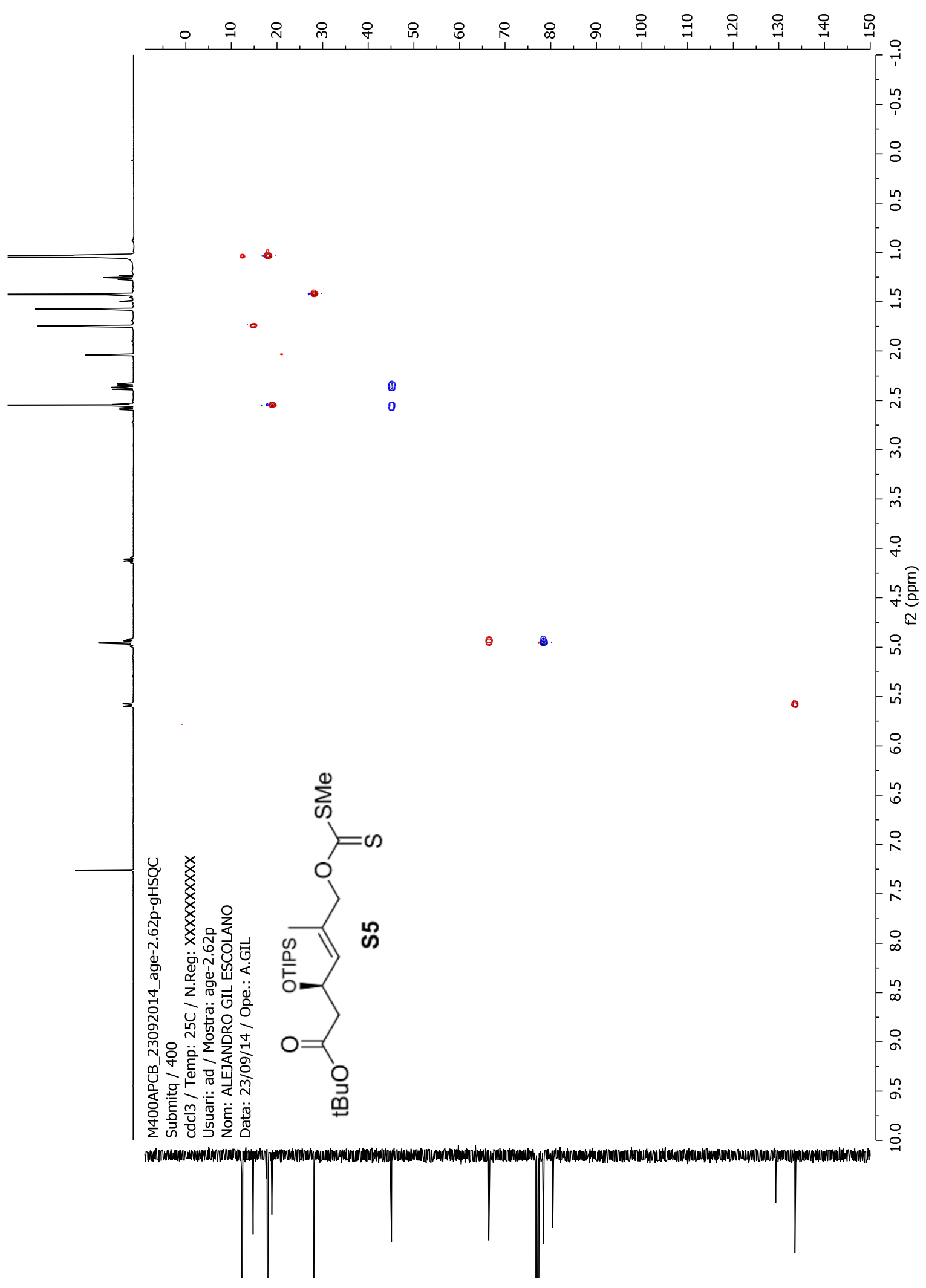




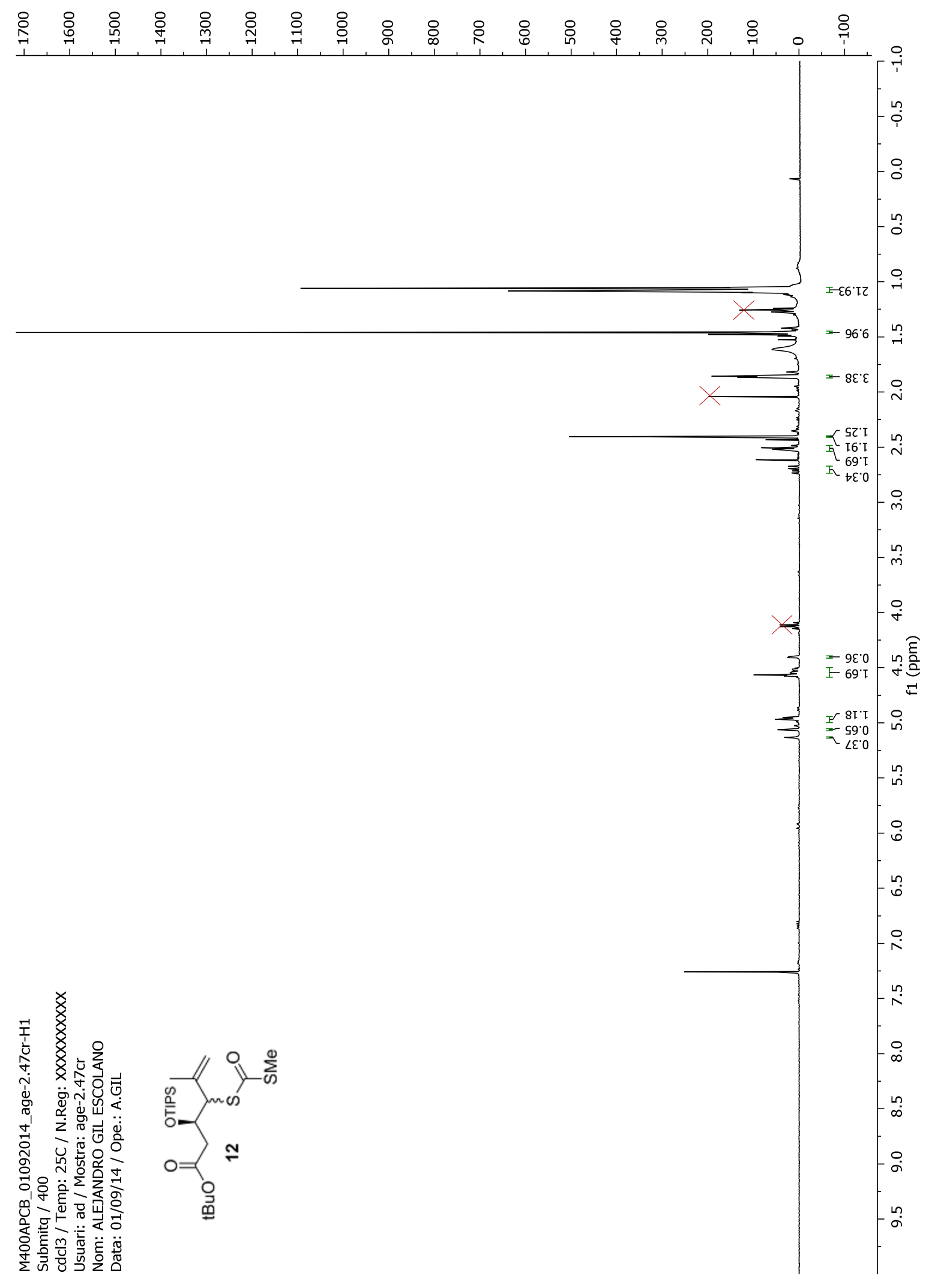




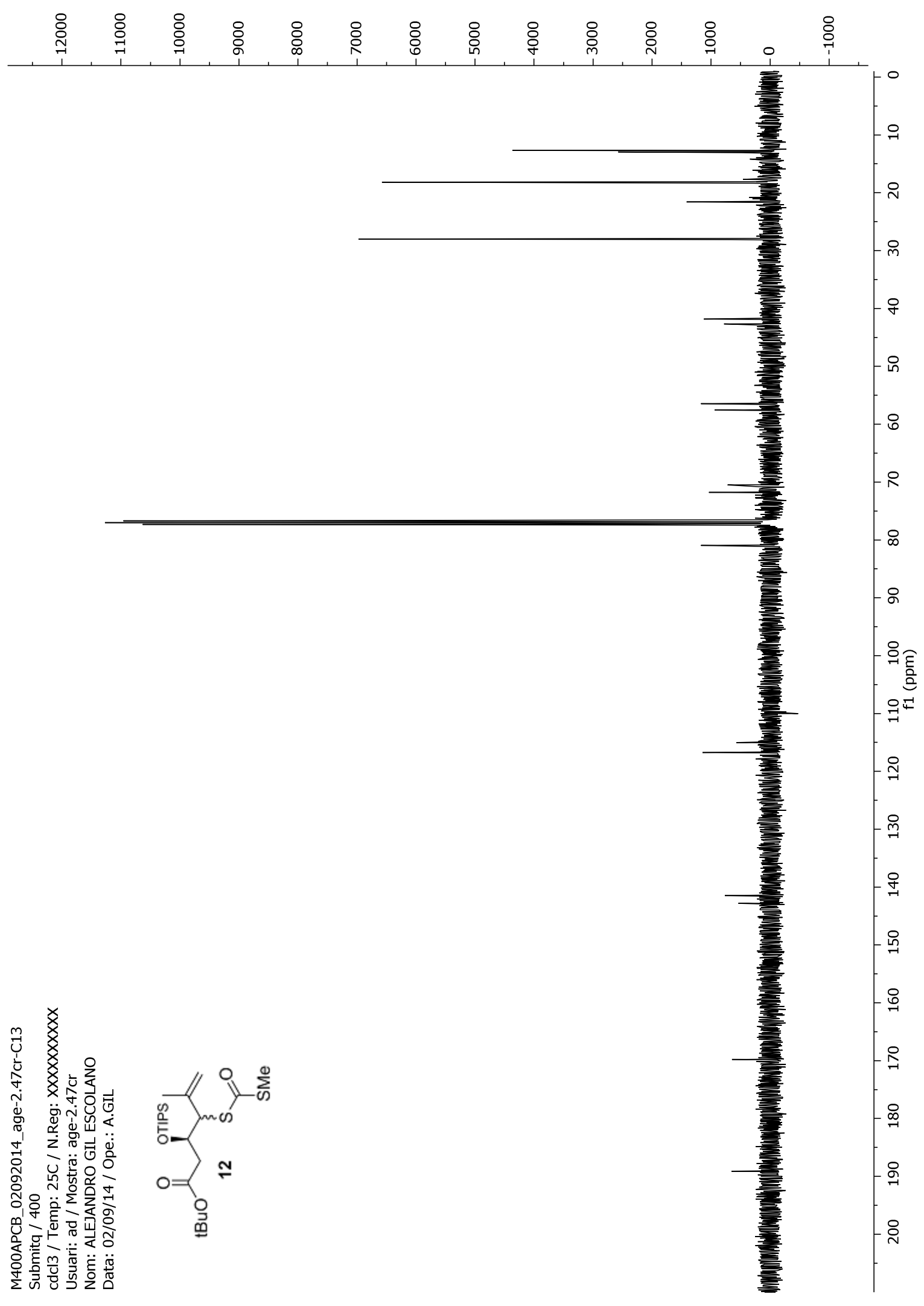


(udd) If

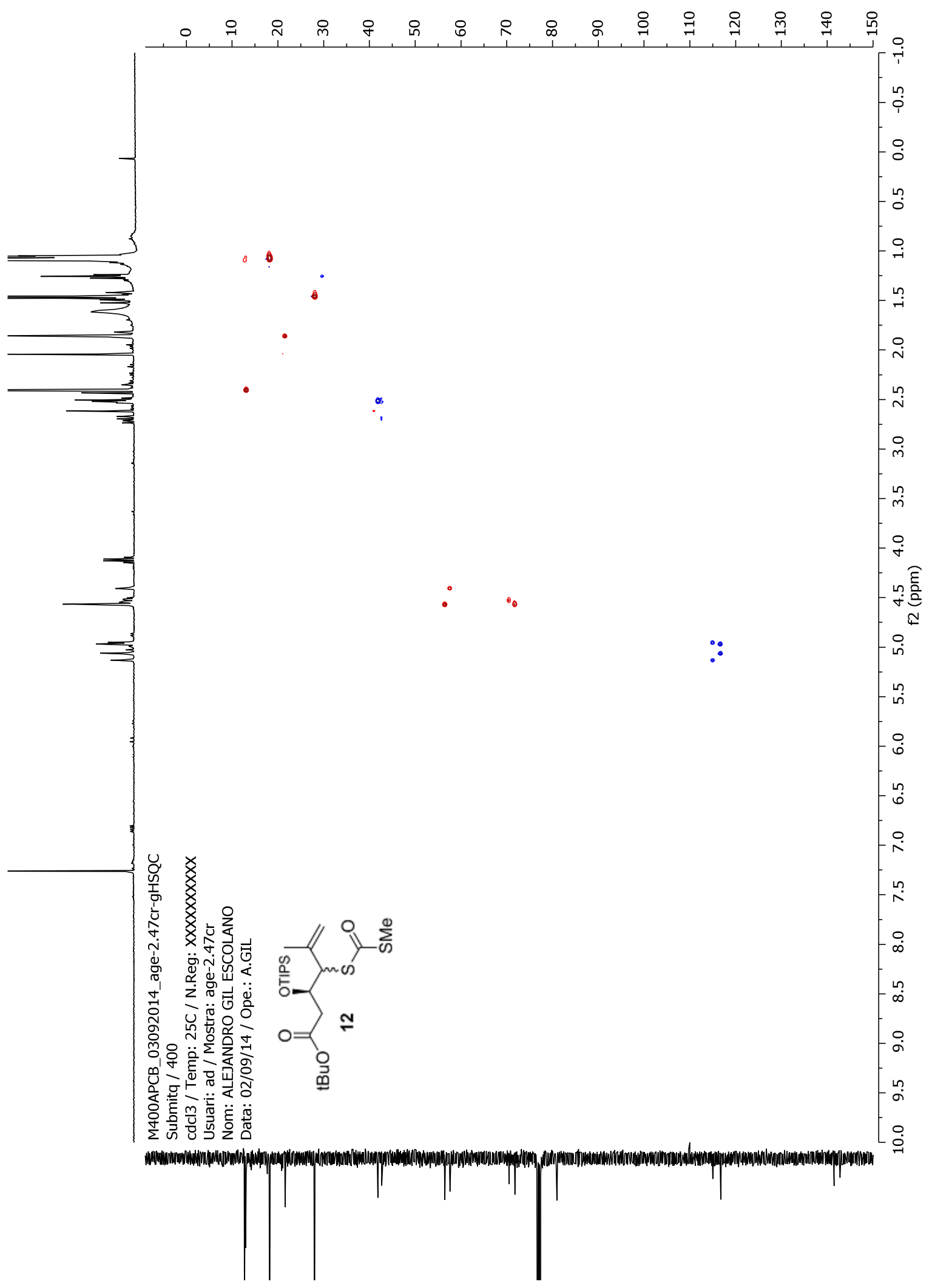




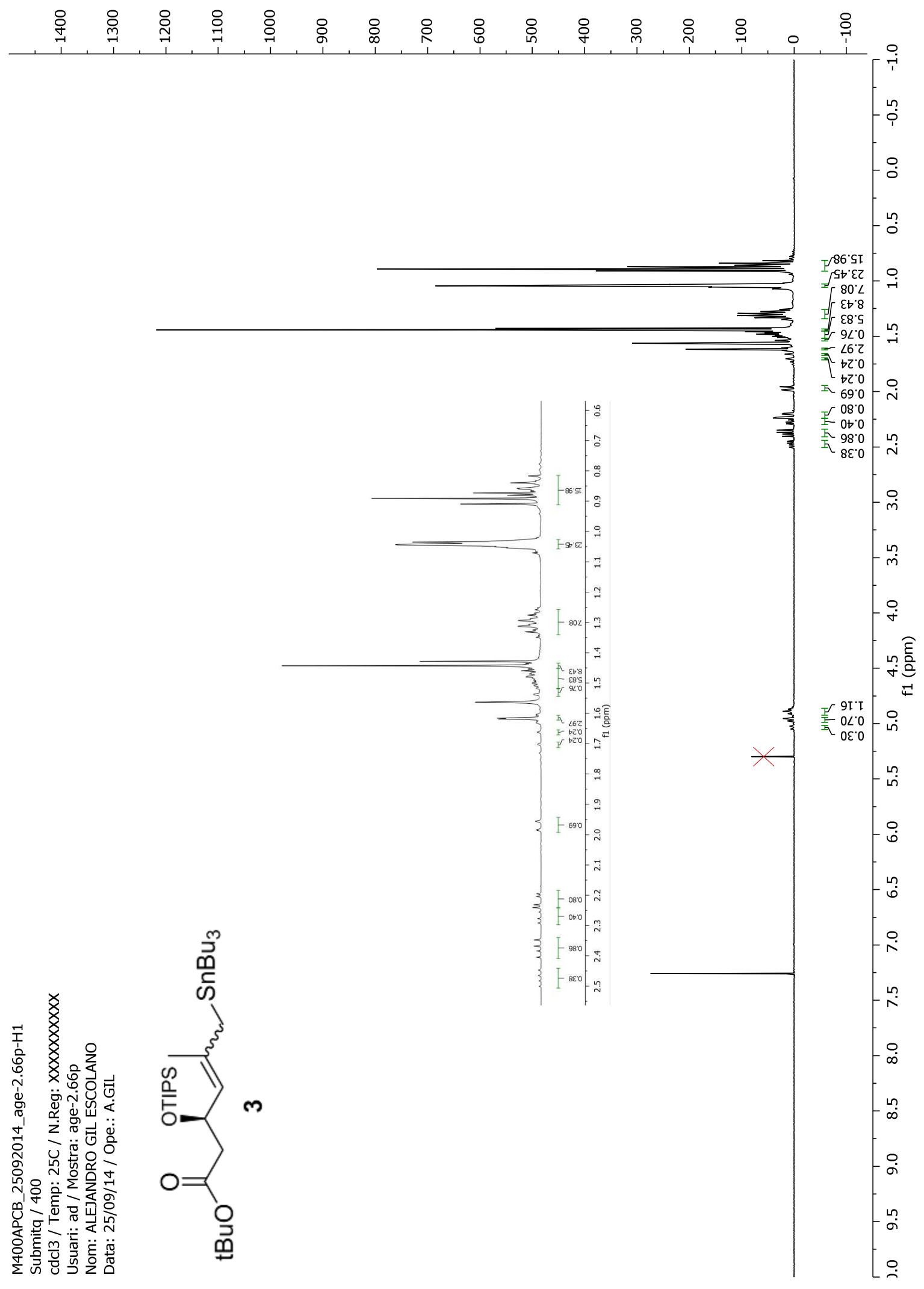


(undd) if

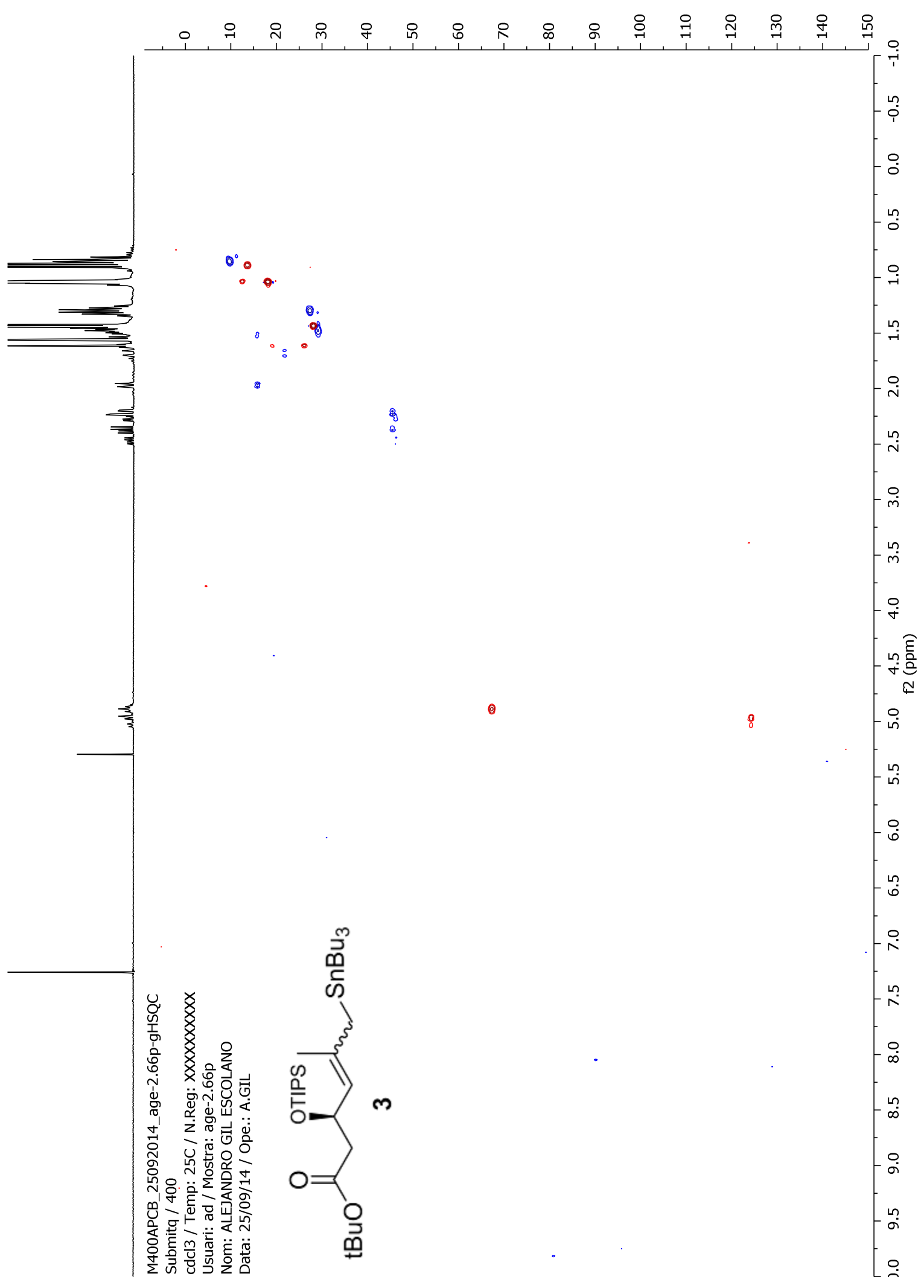




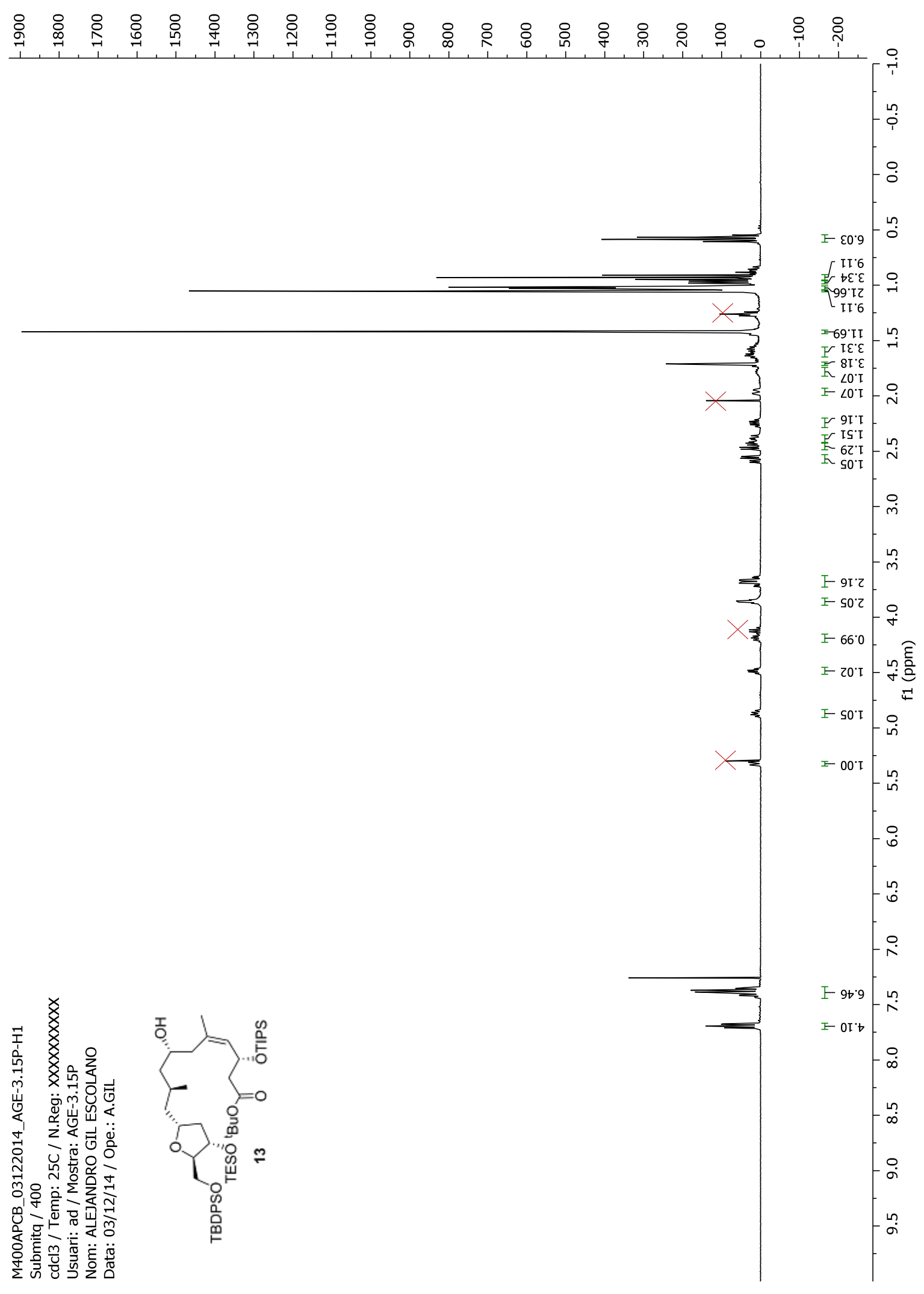




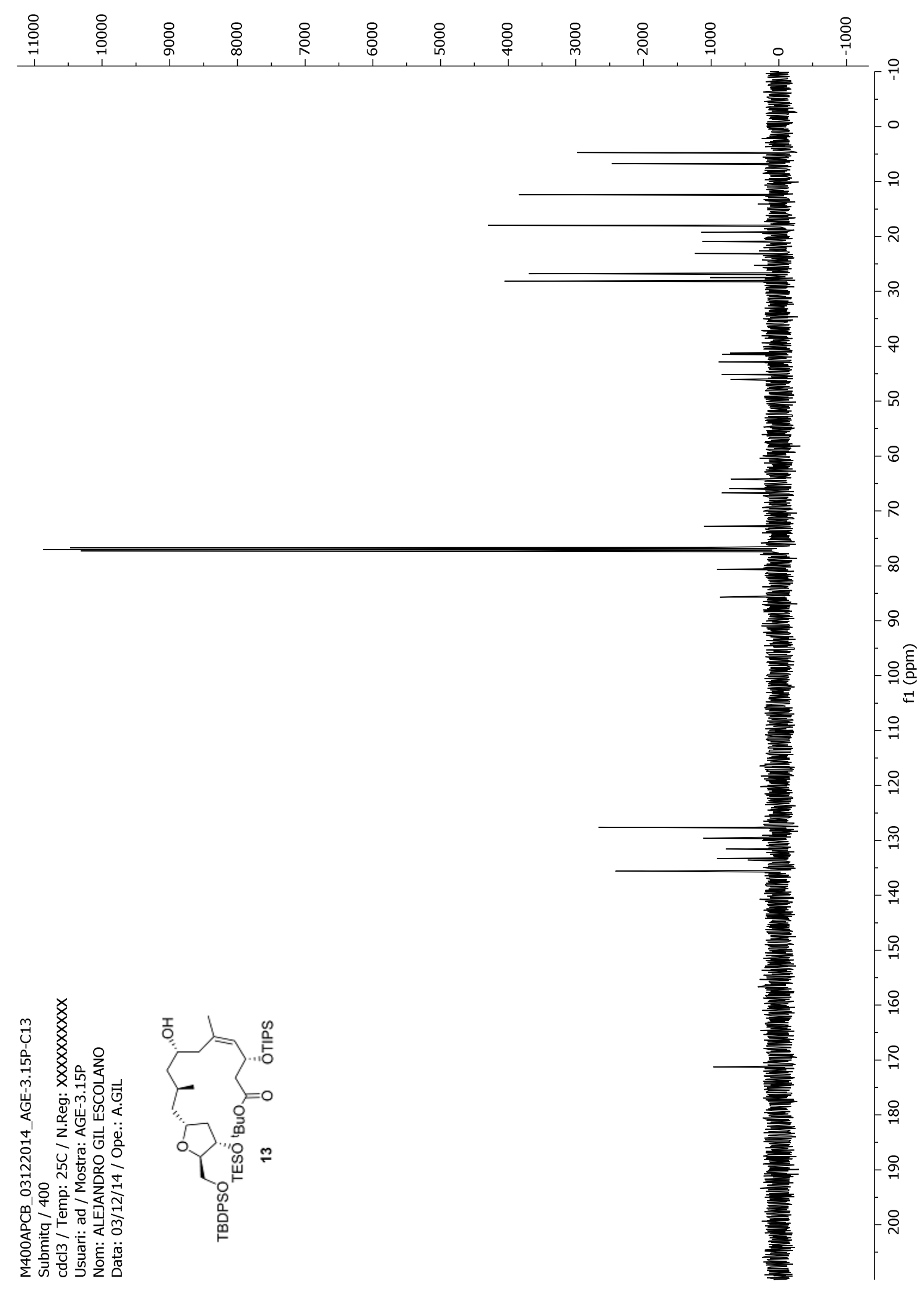


(udd) If

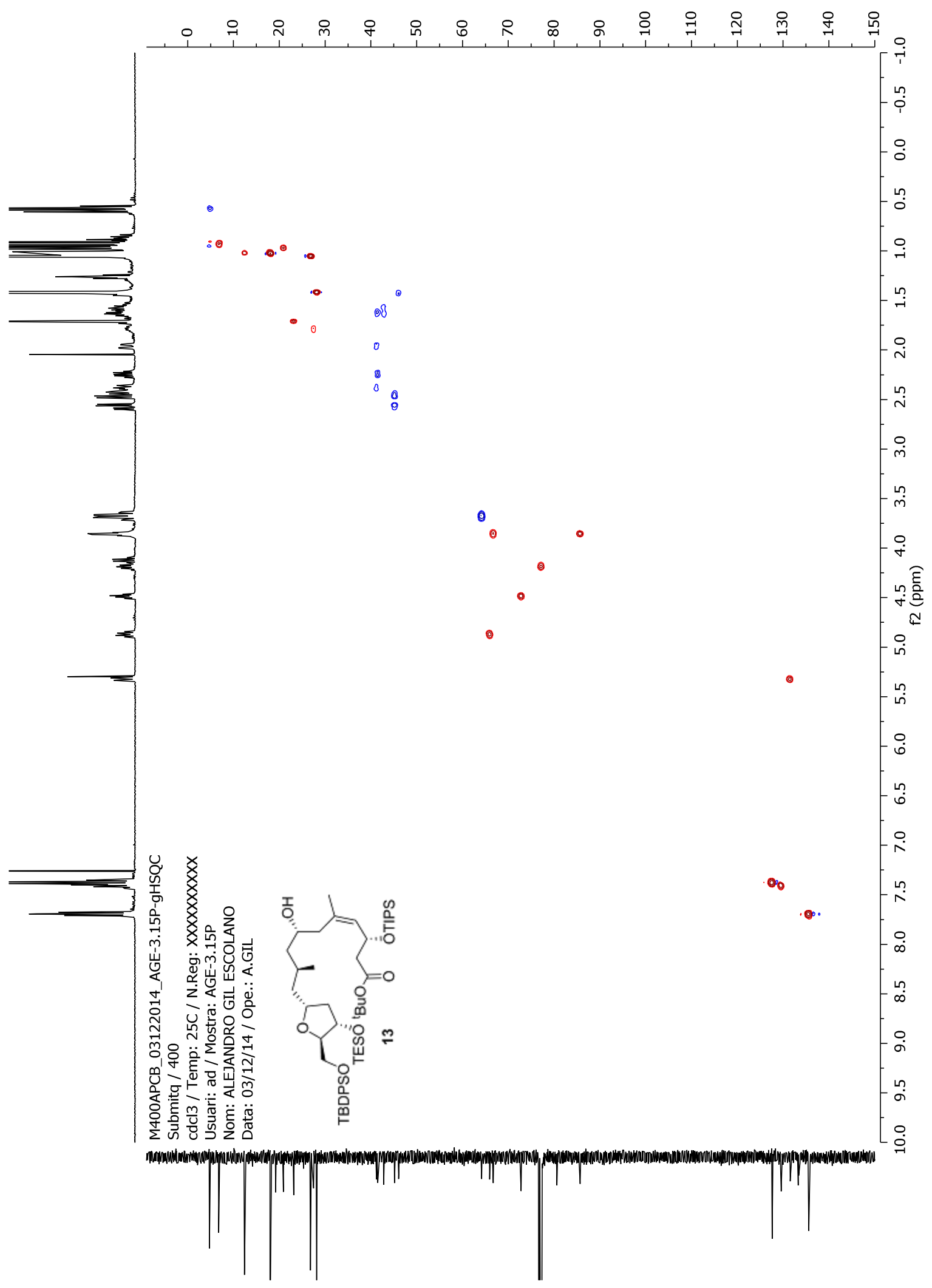




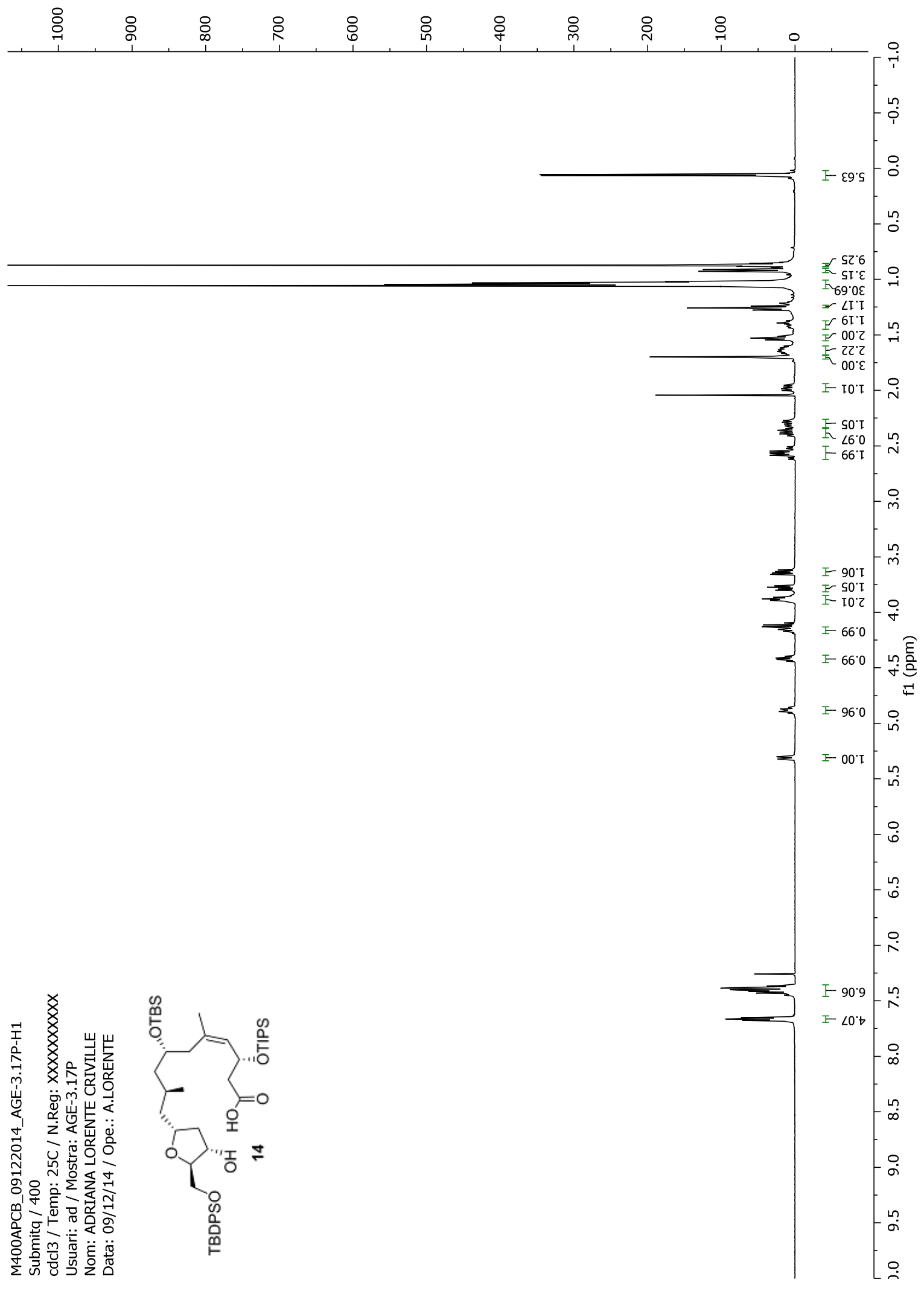




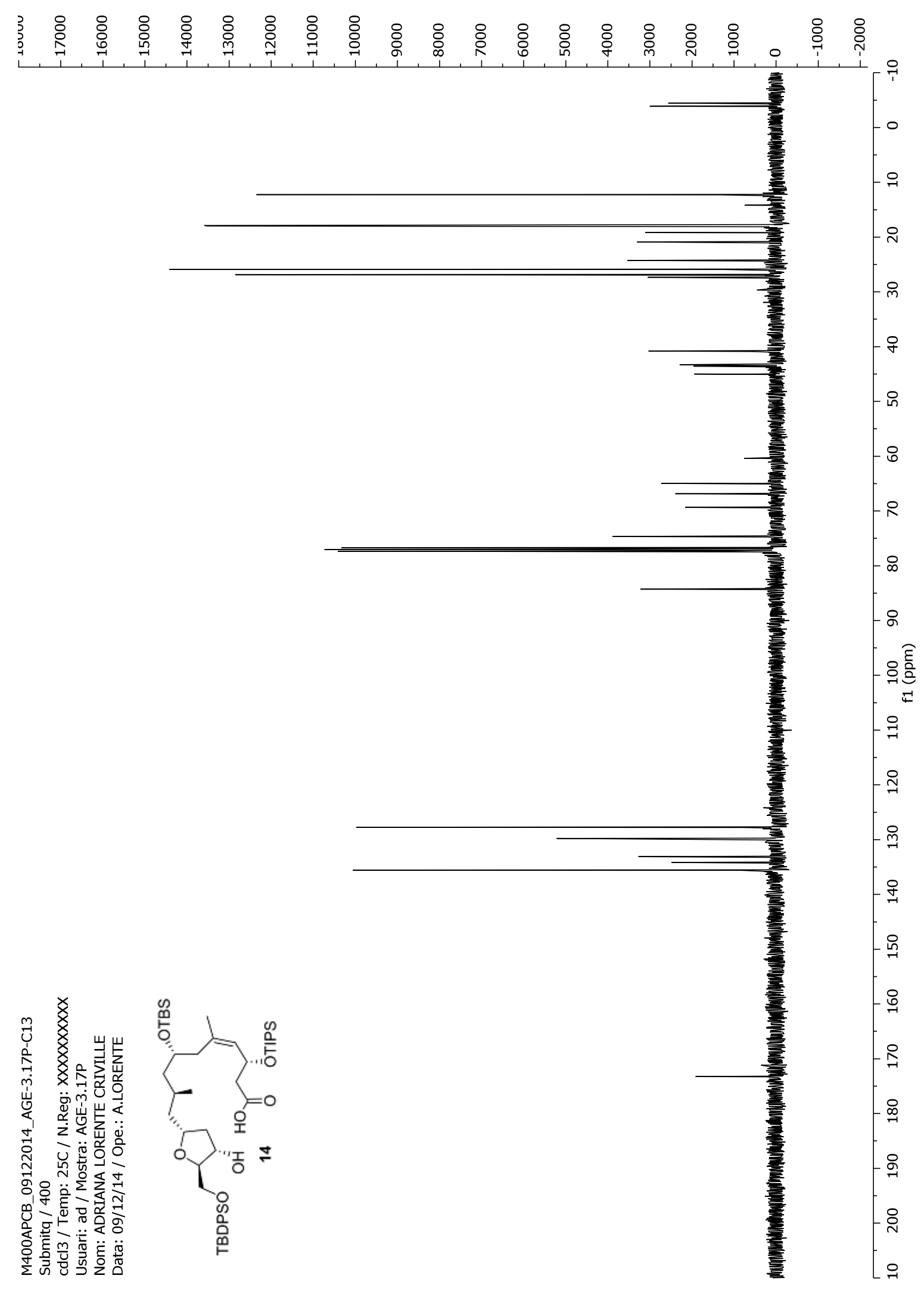


(udd) If

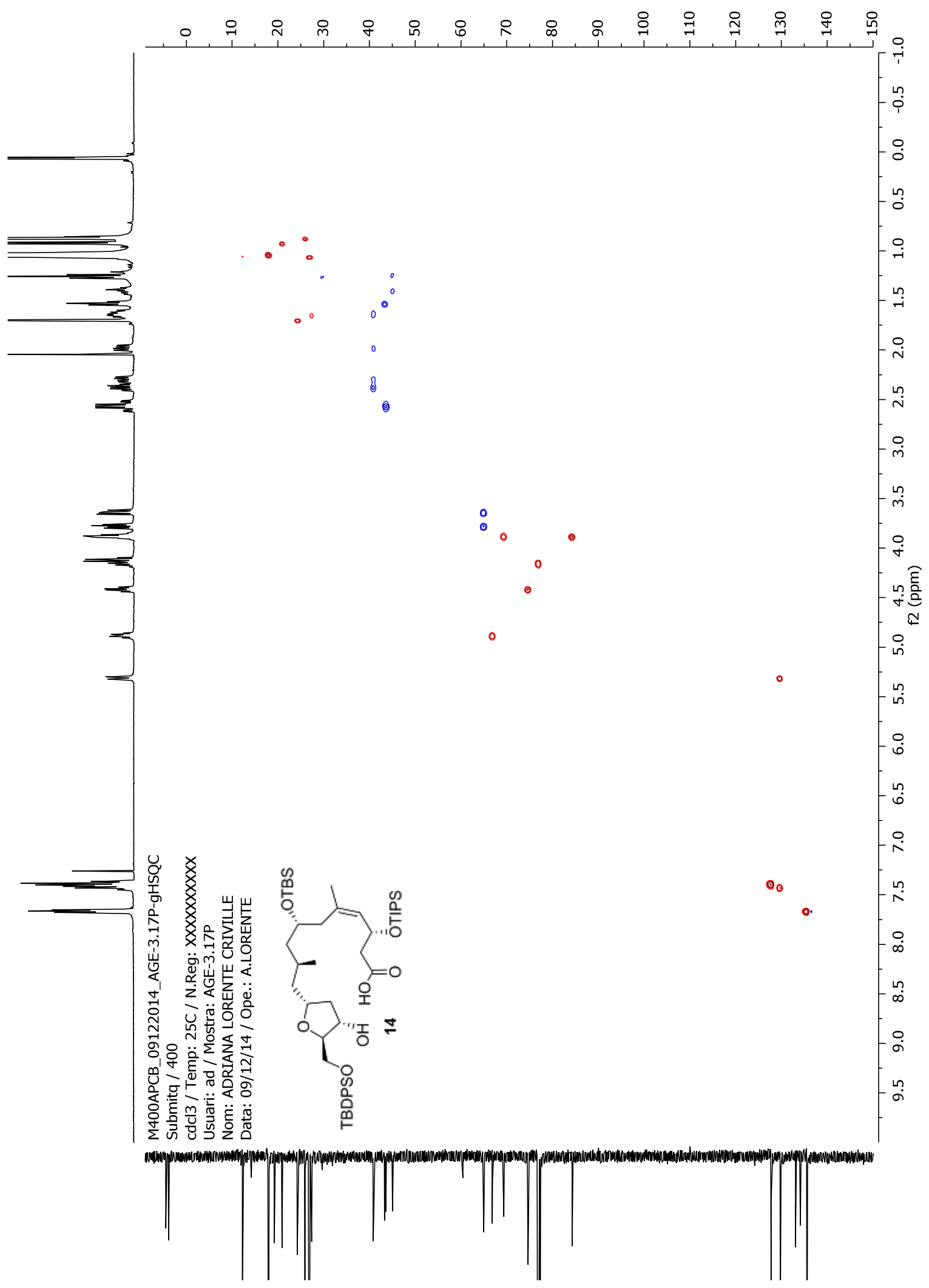




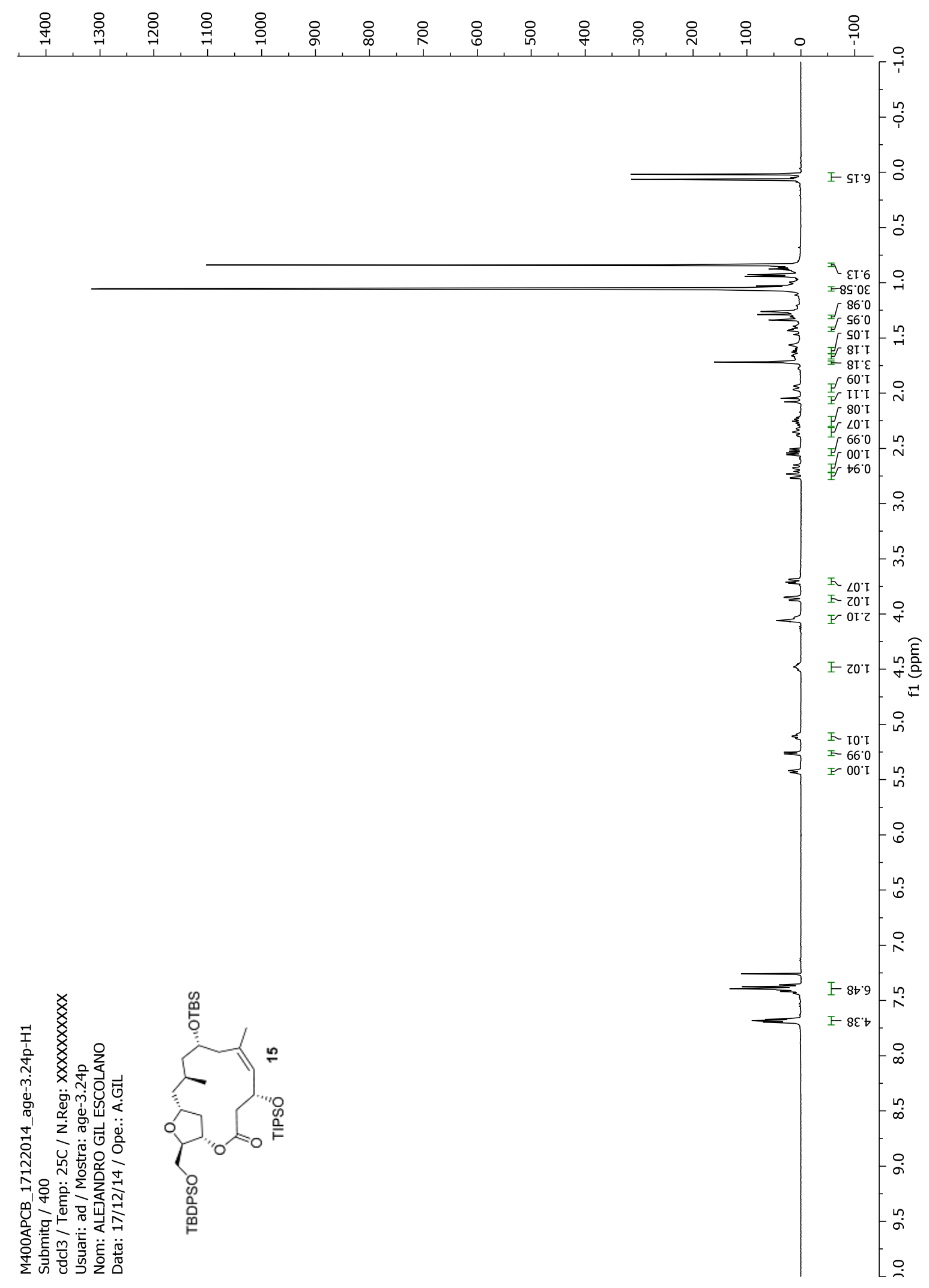




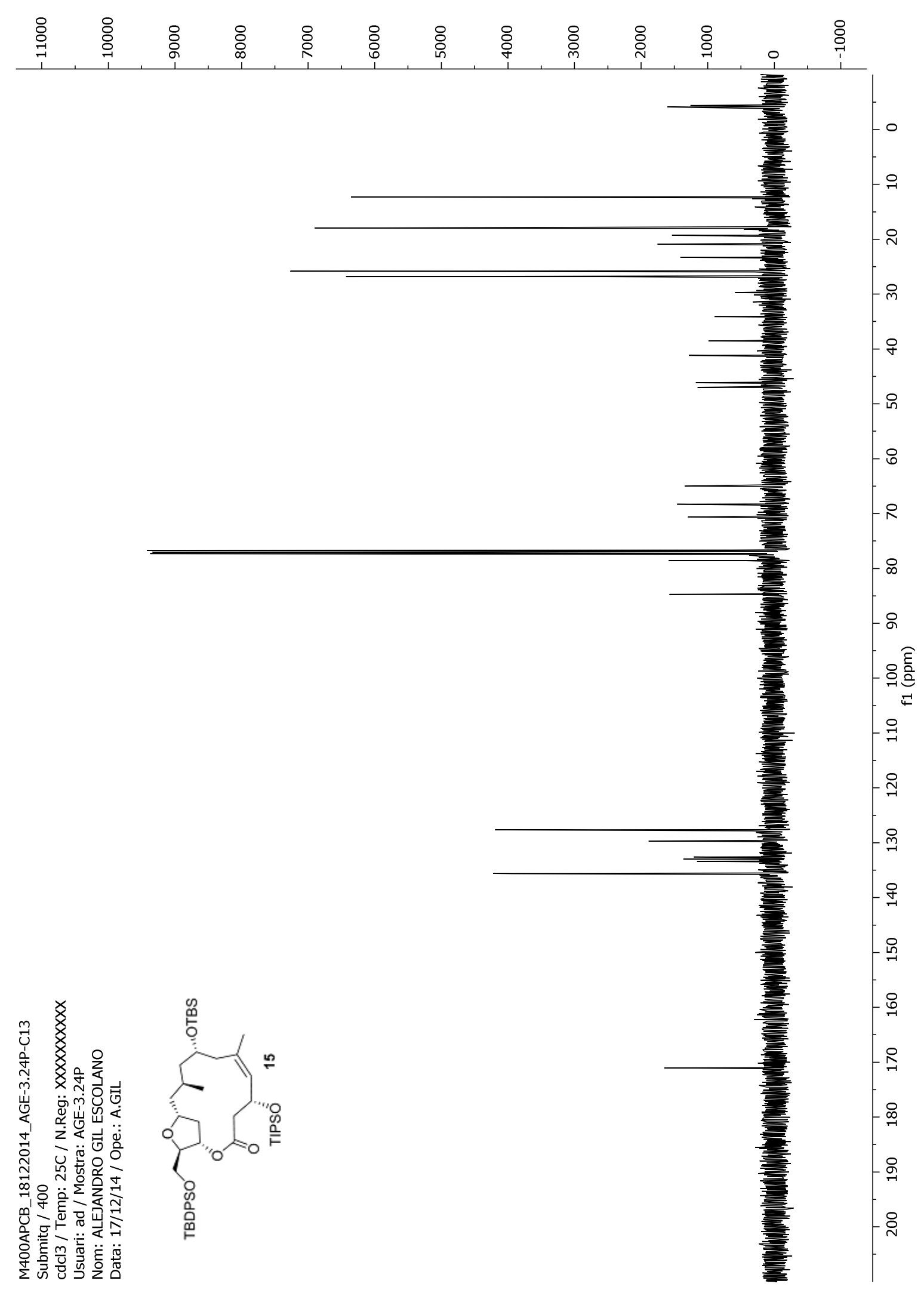


(udd) If

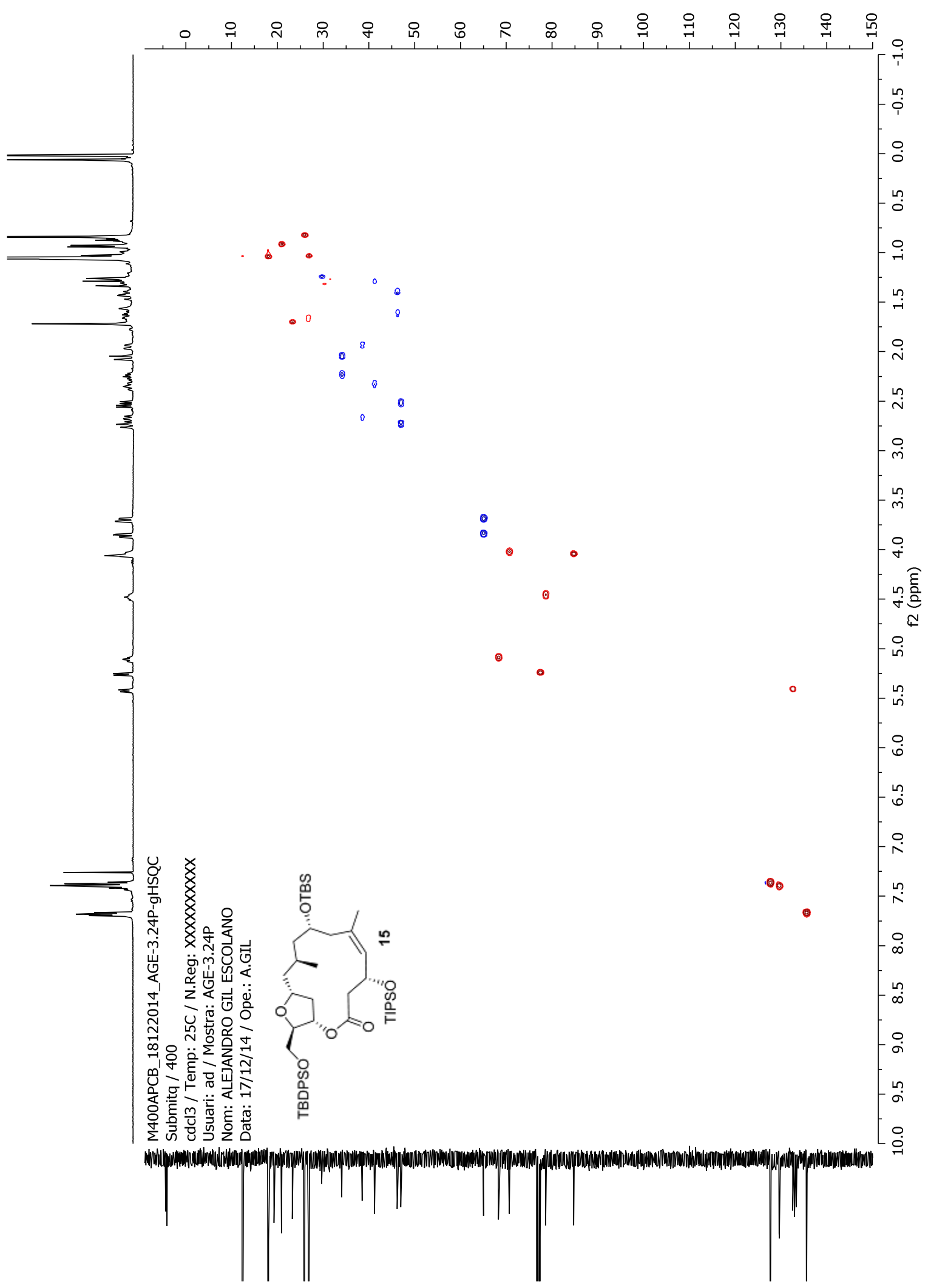




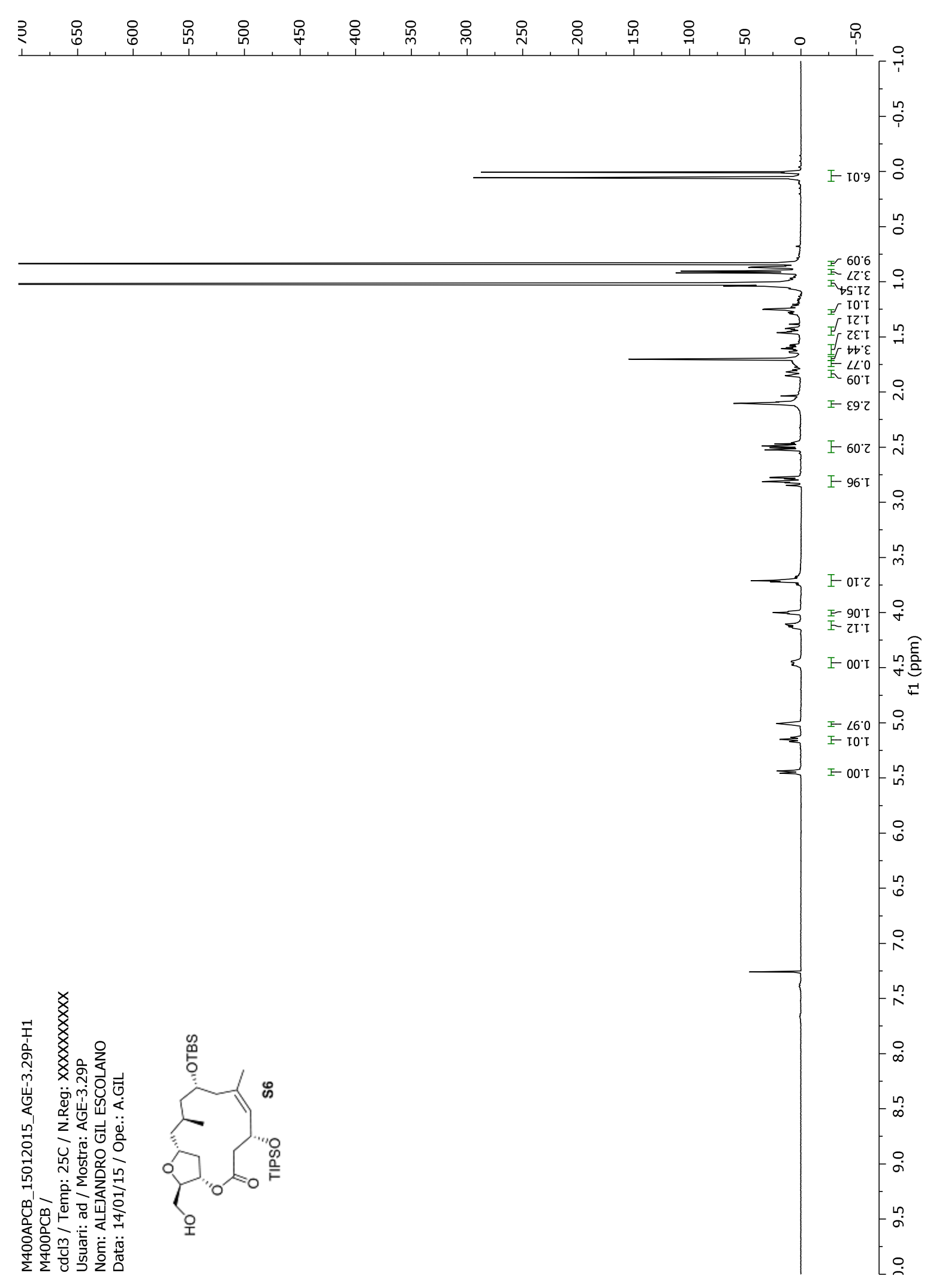




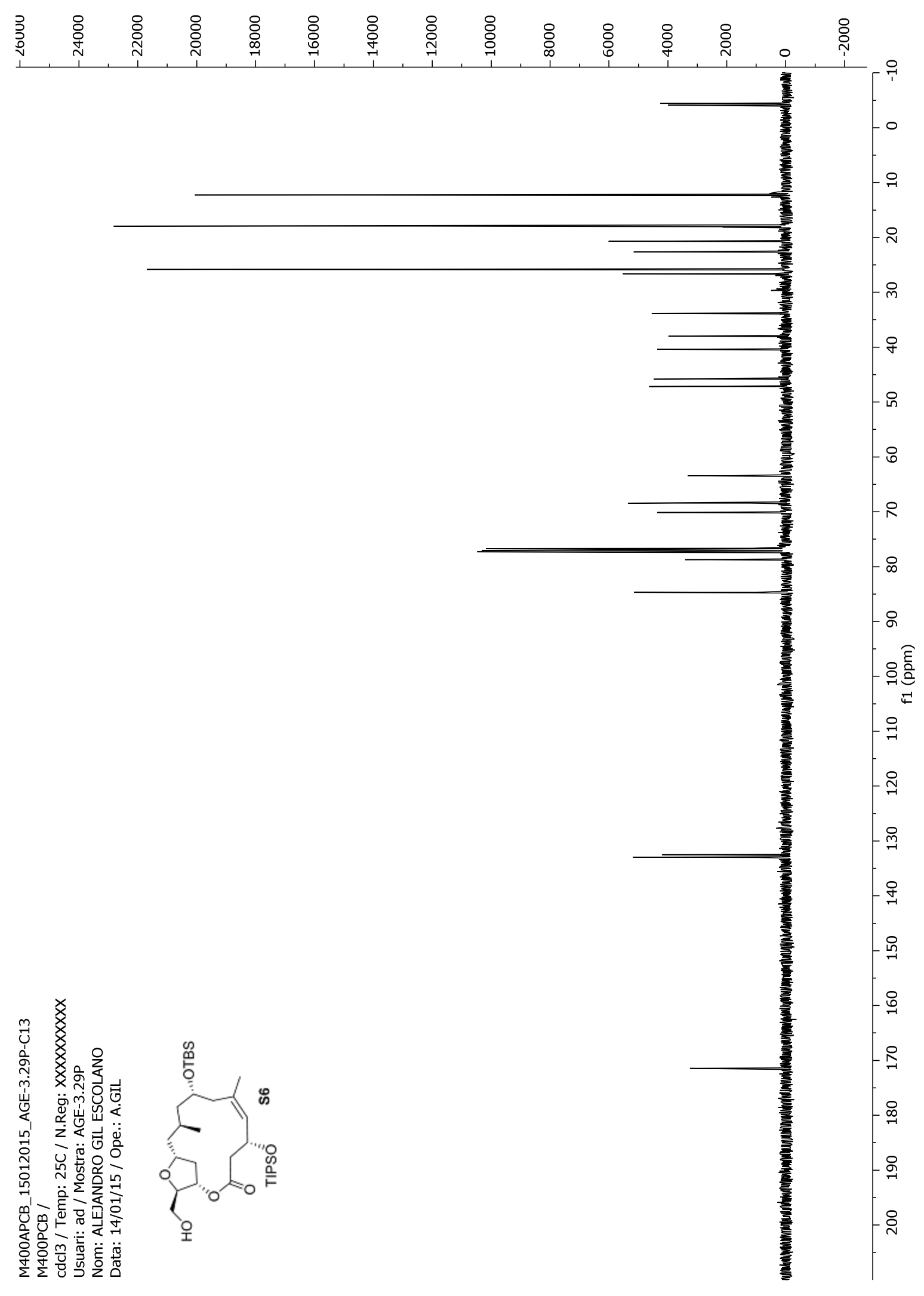


(udd) If

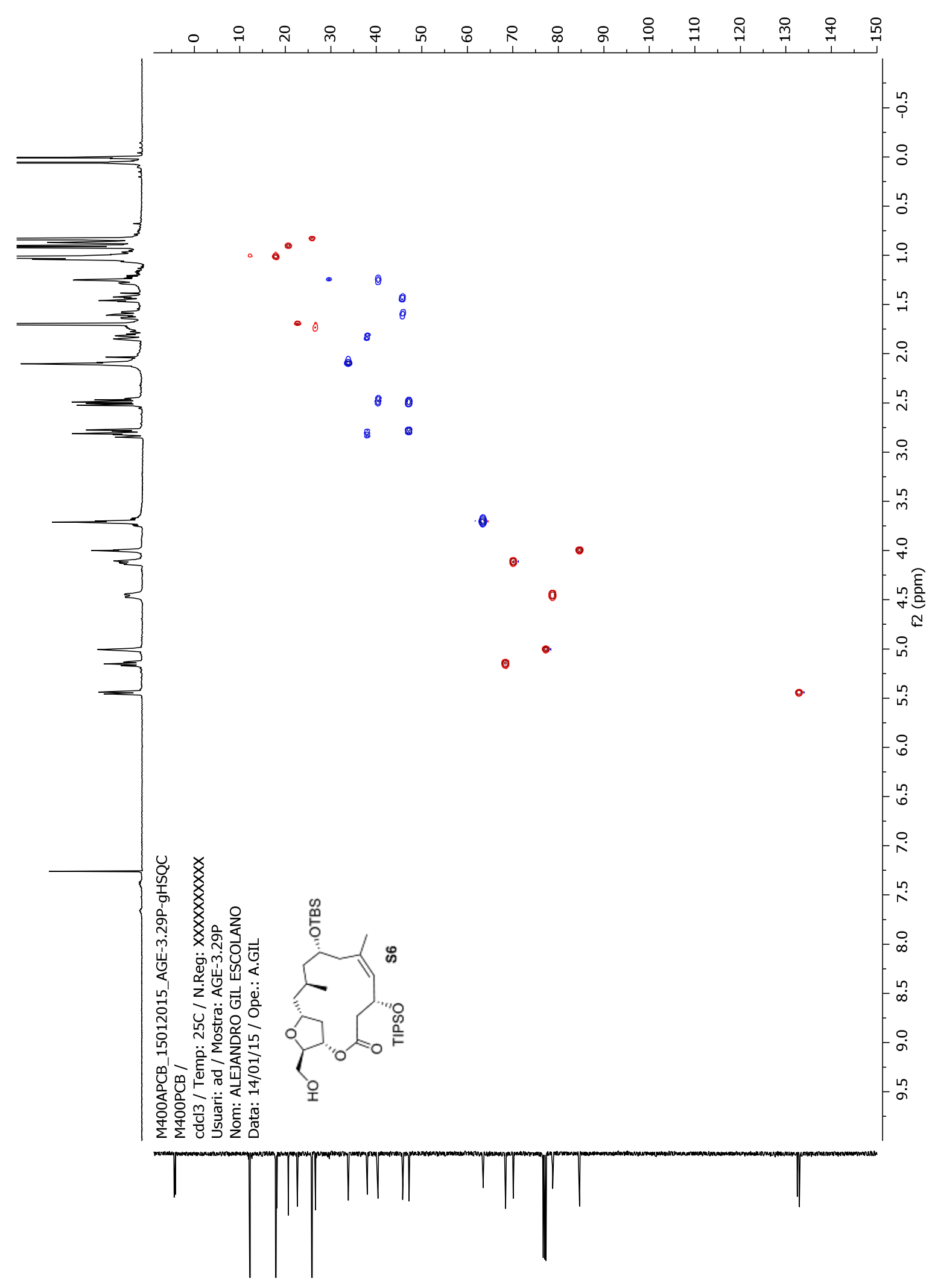




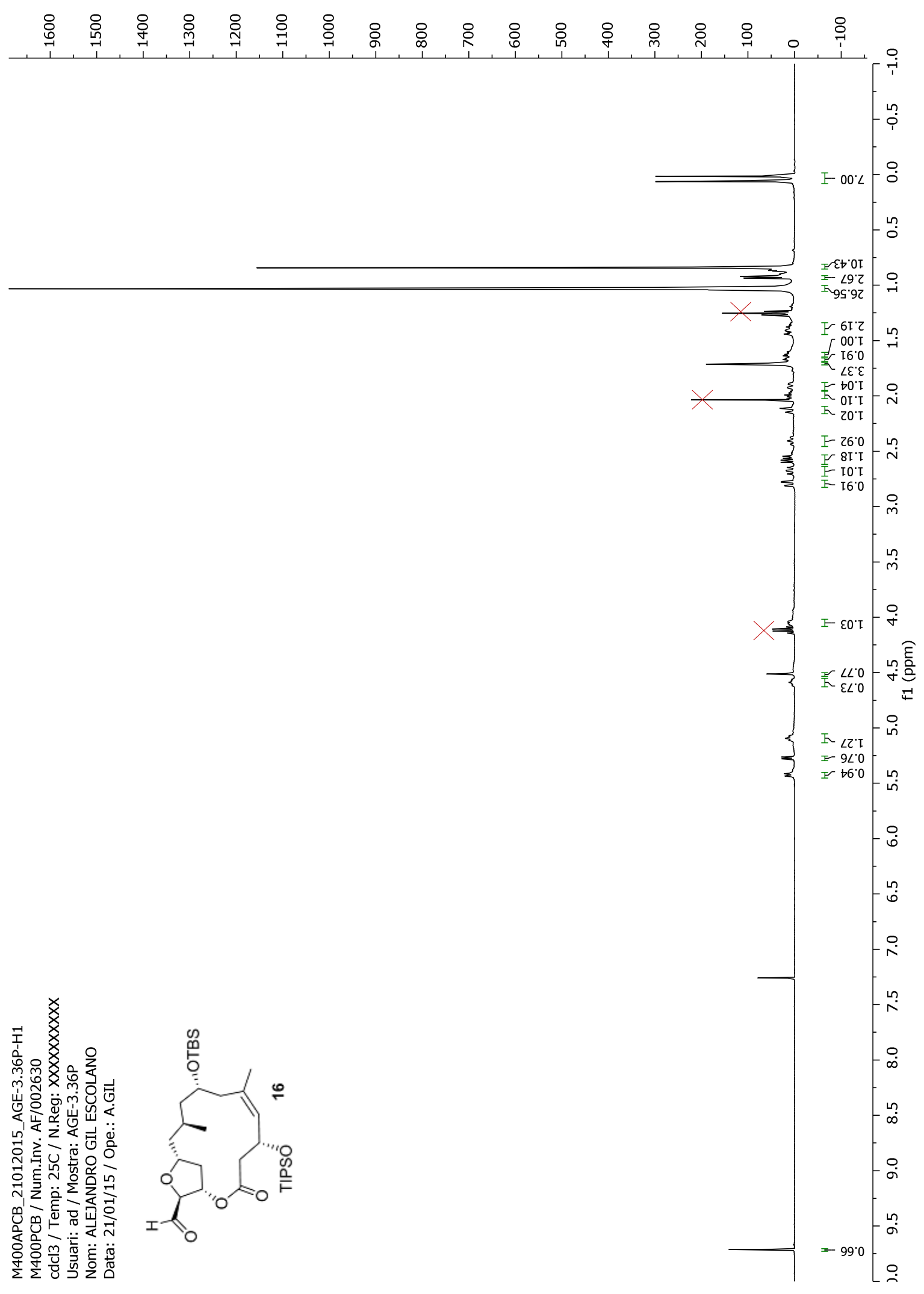




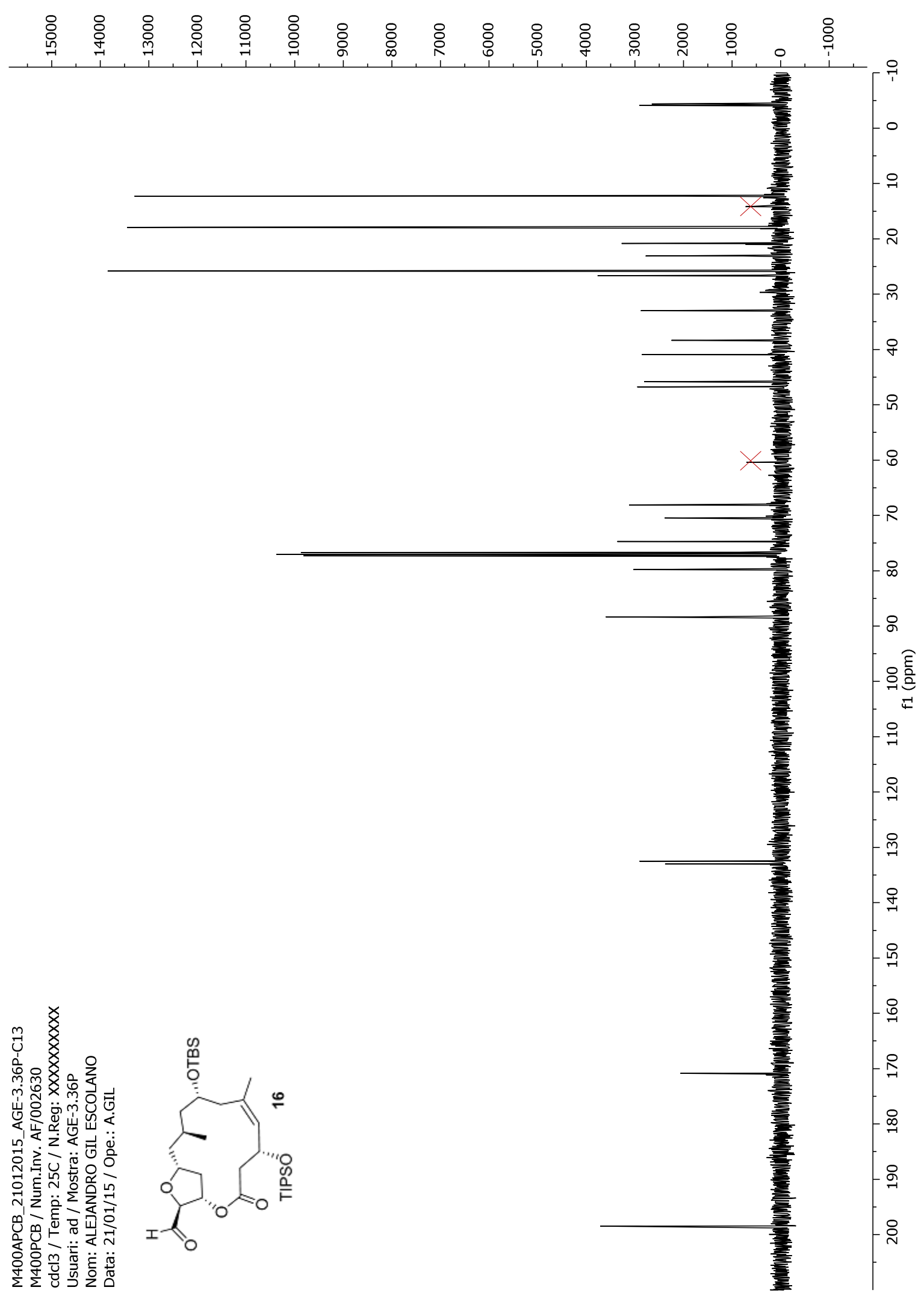


(udd) If

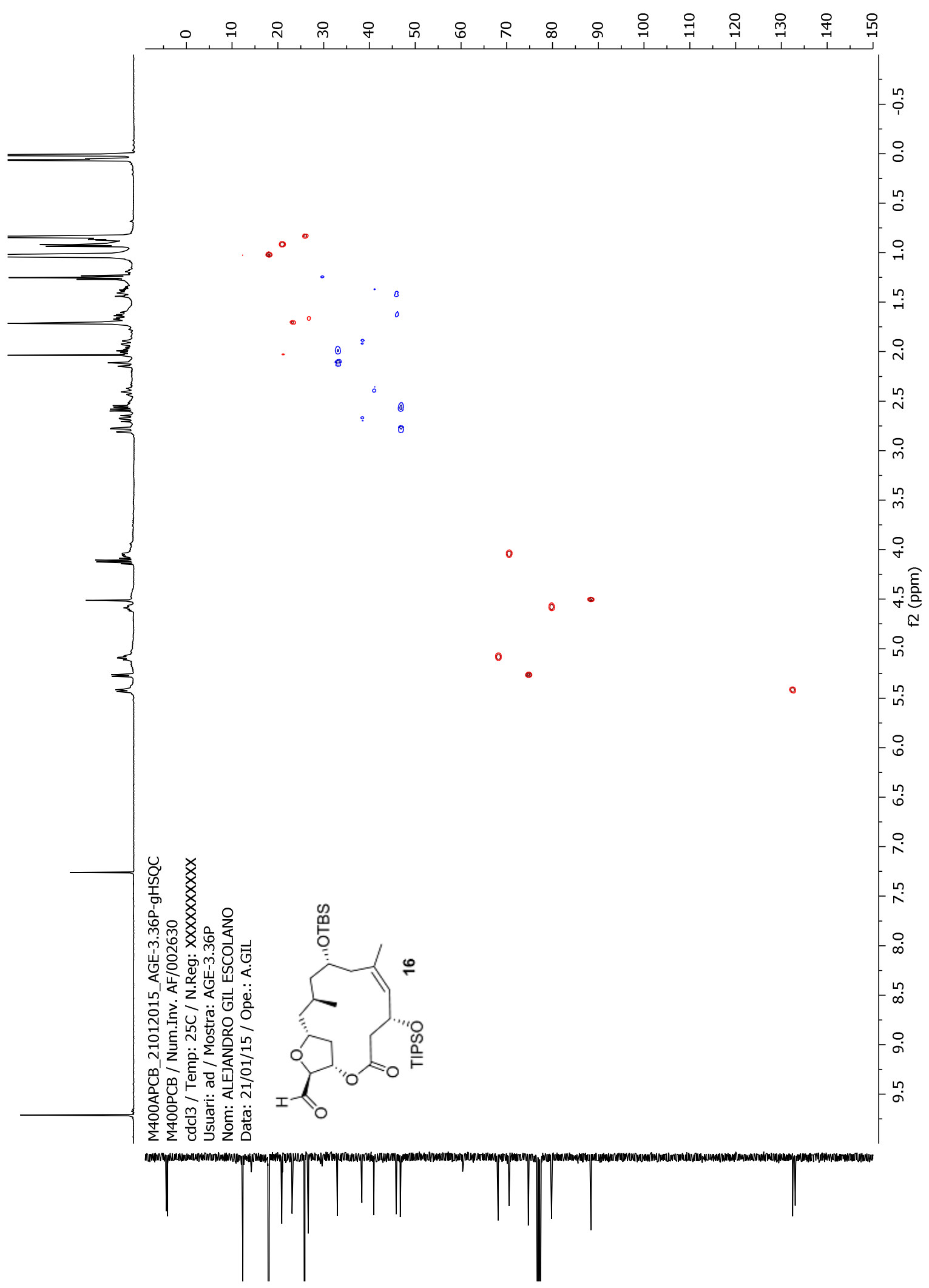




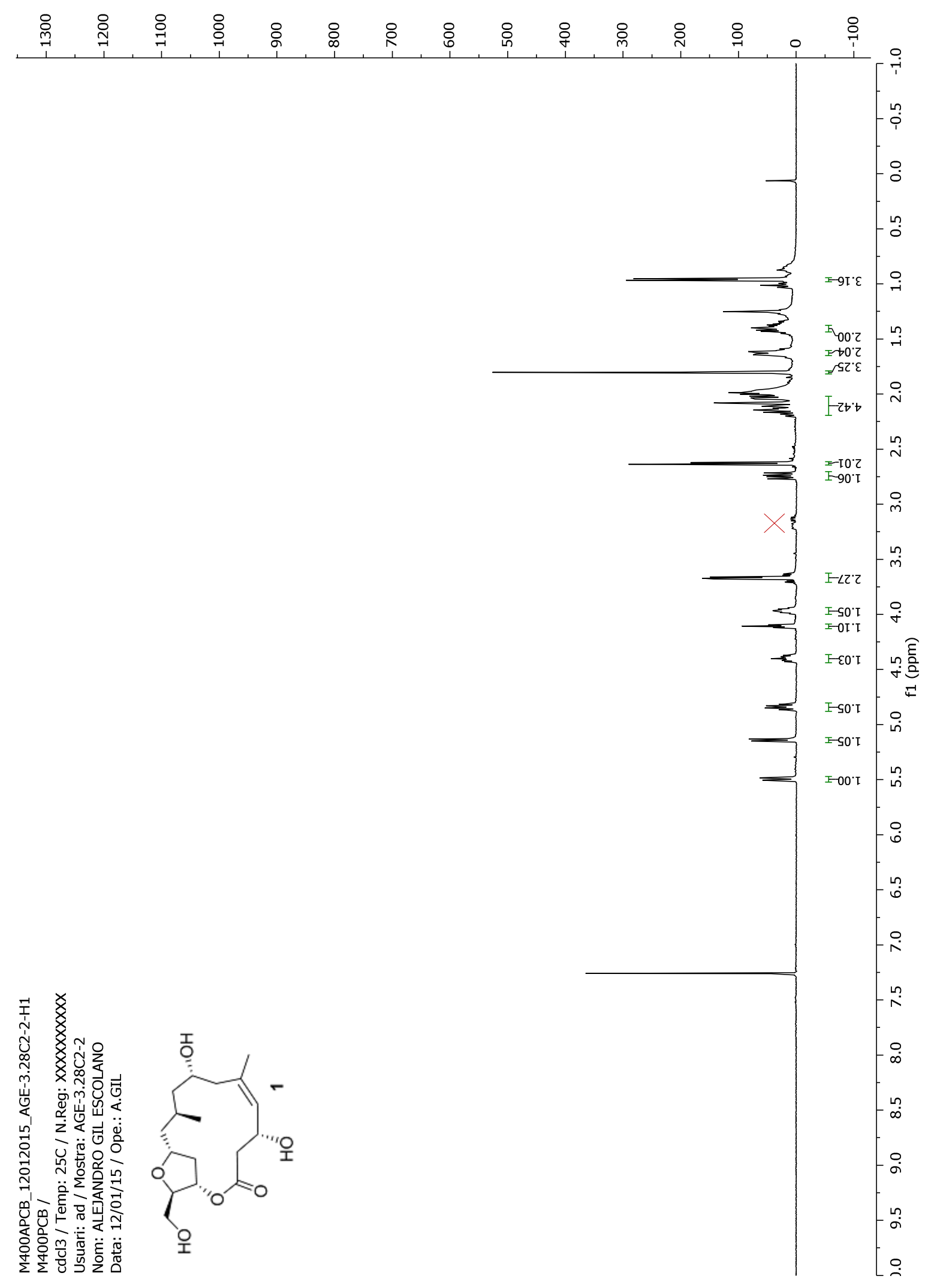




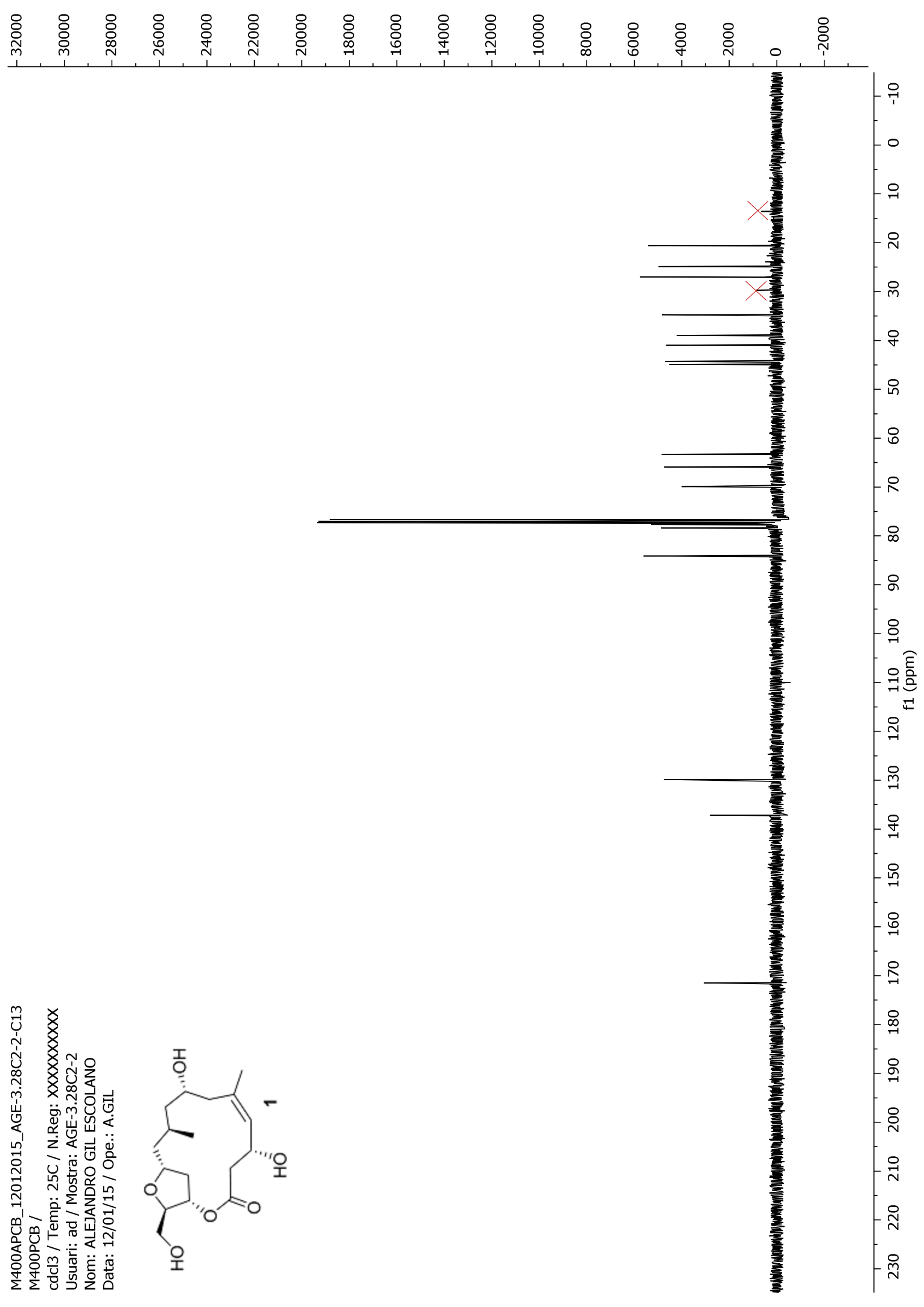


(udd) if

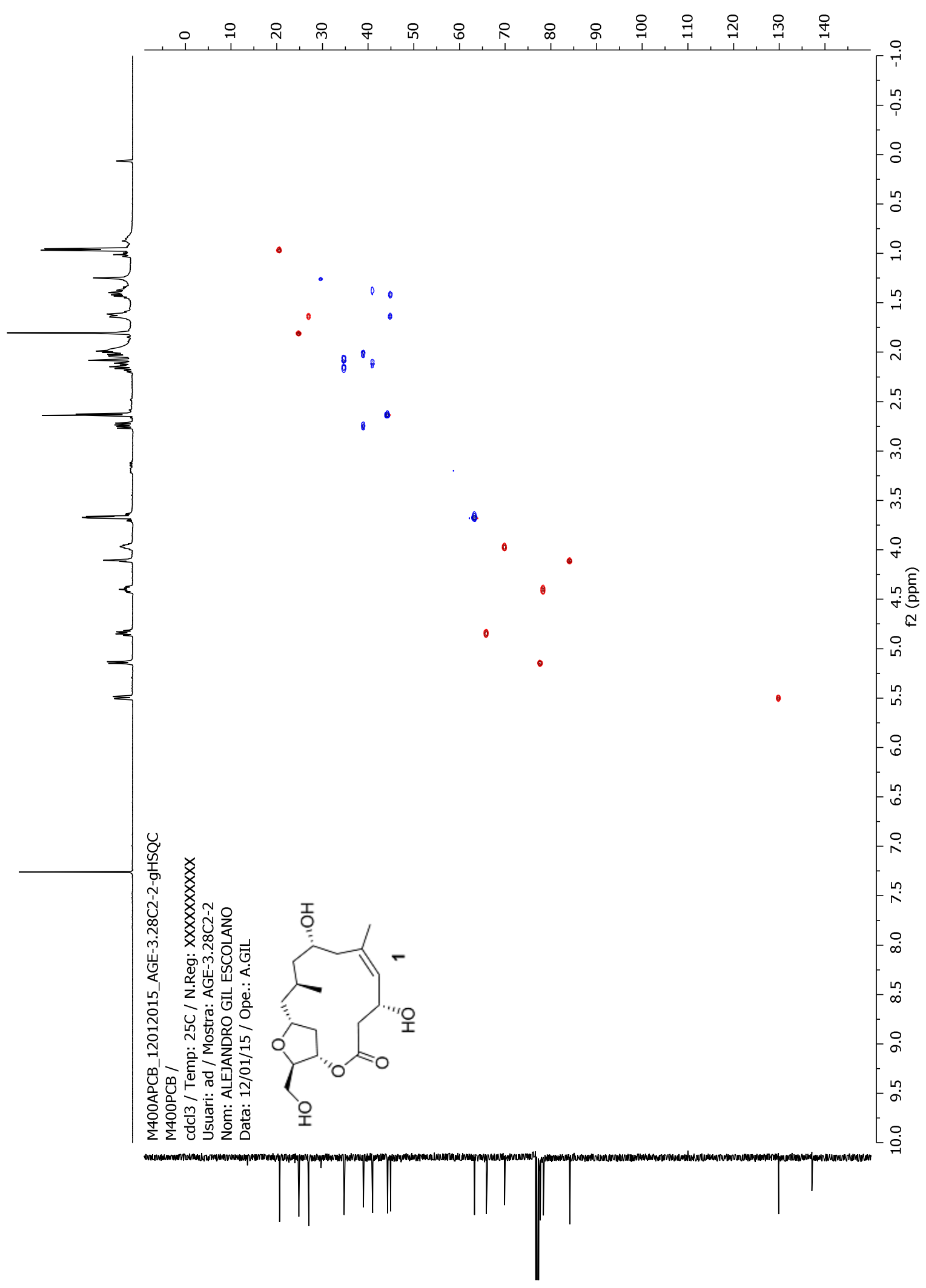




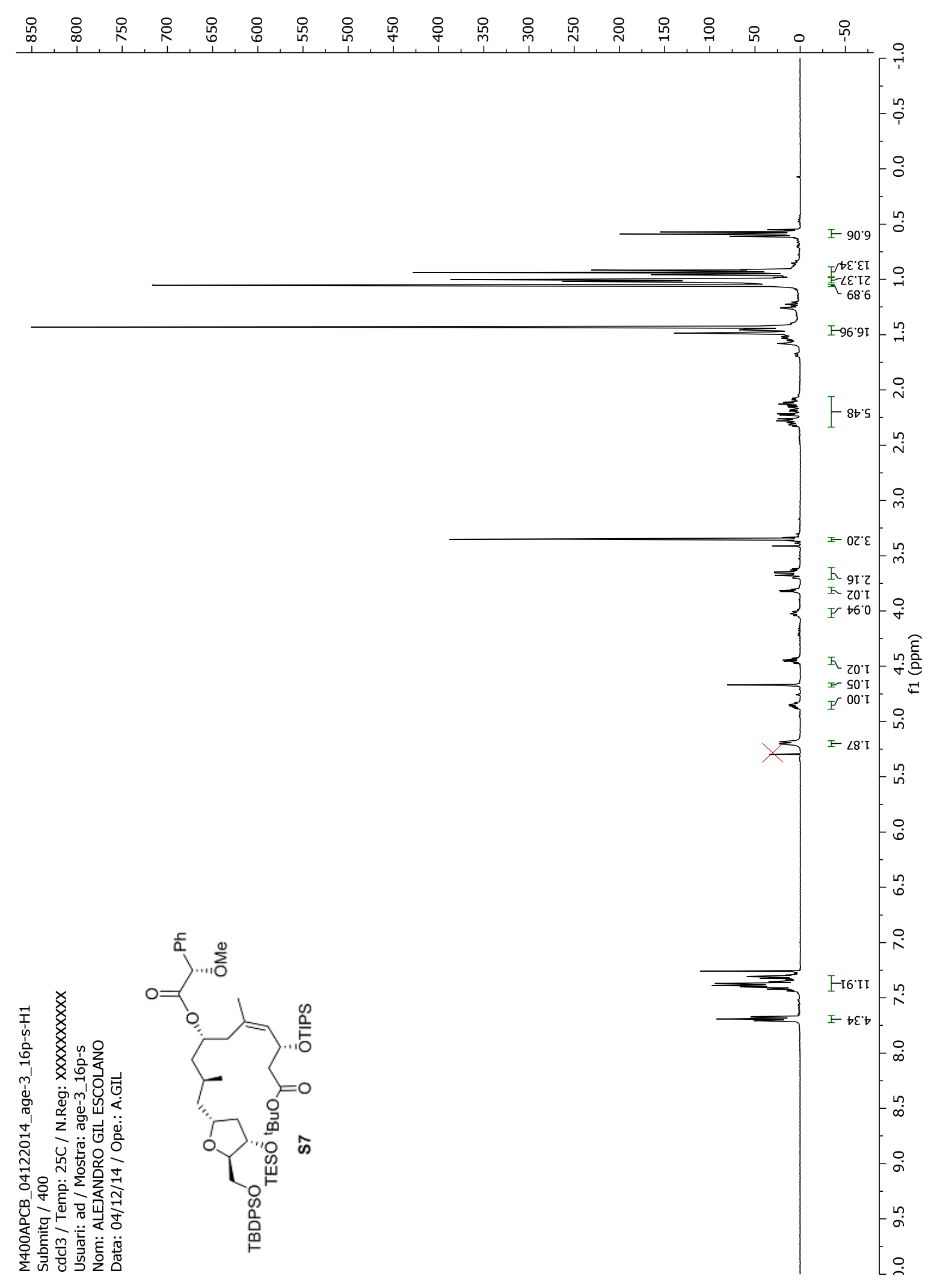


(udd) If

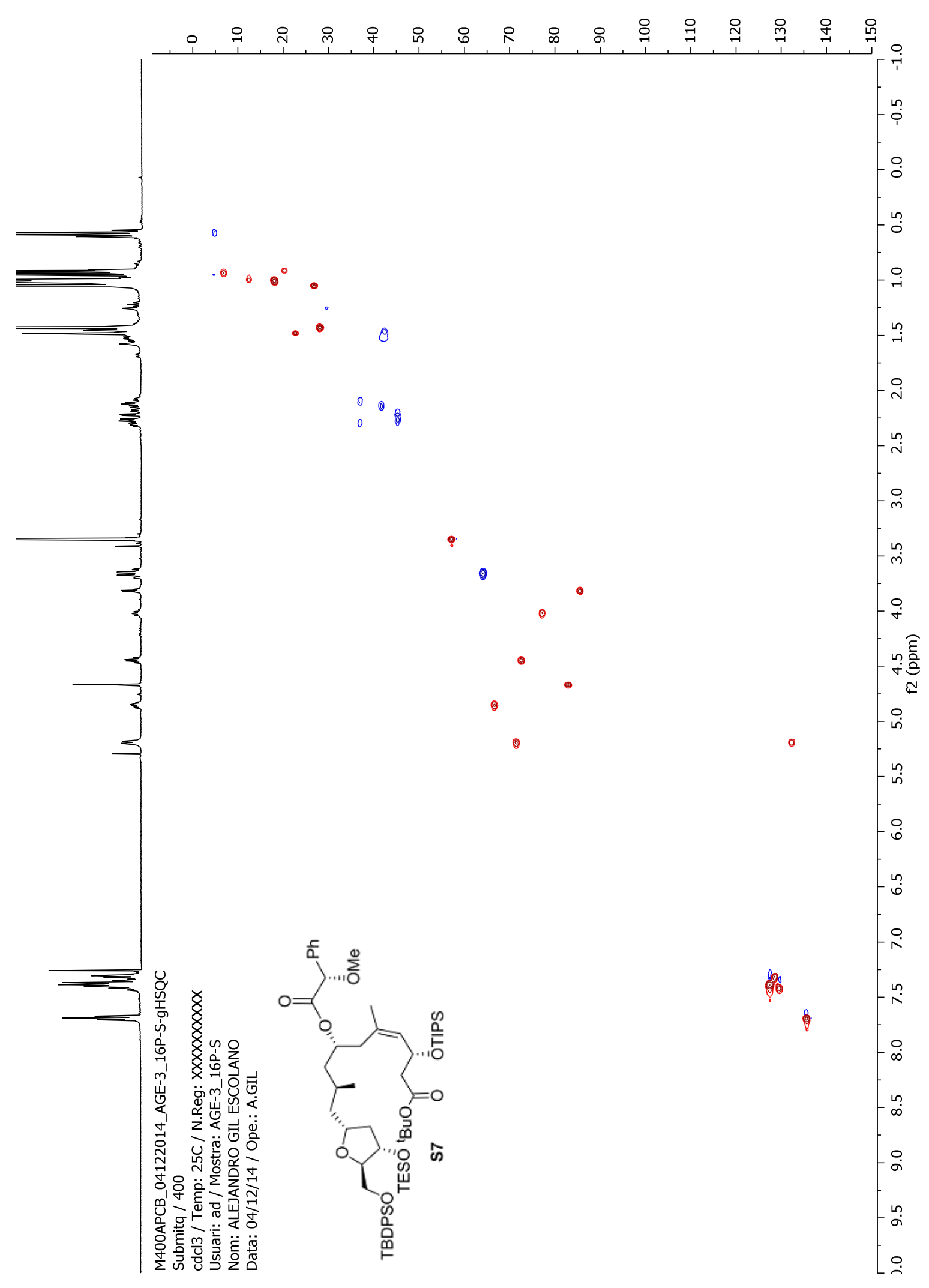


(udd) If

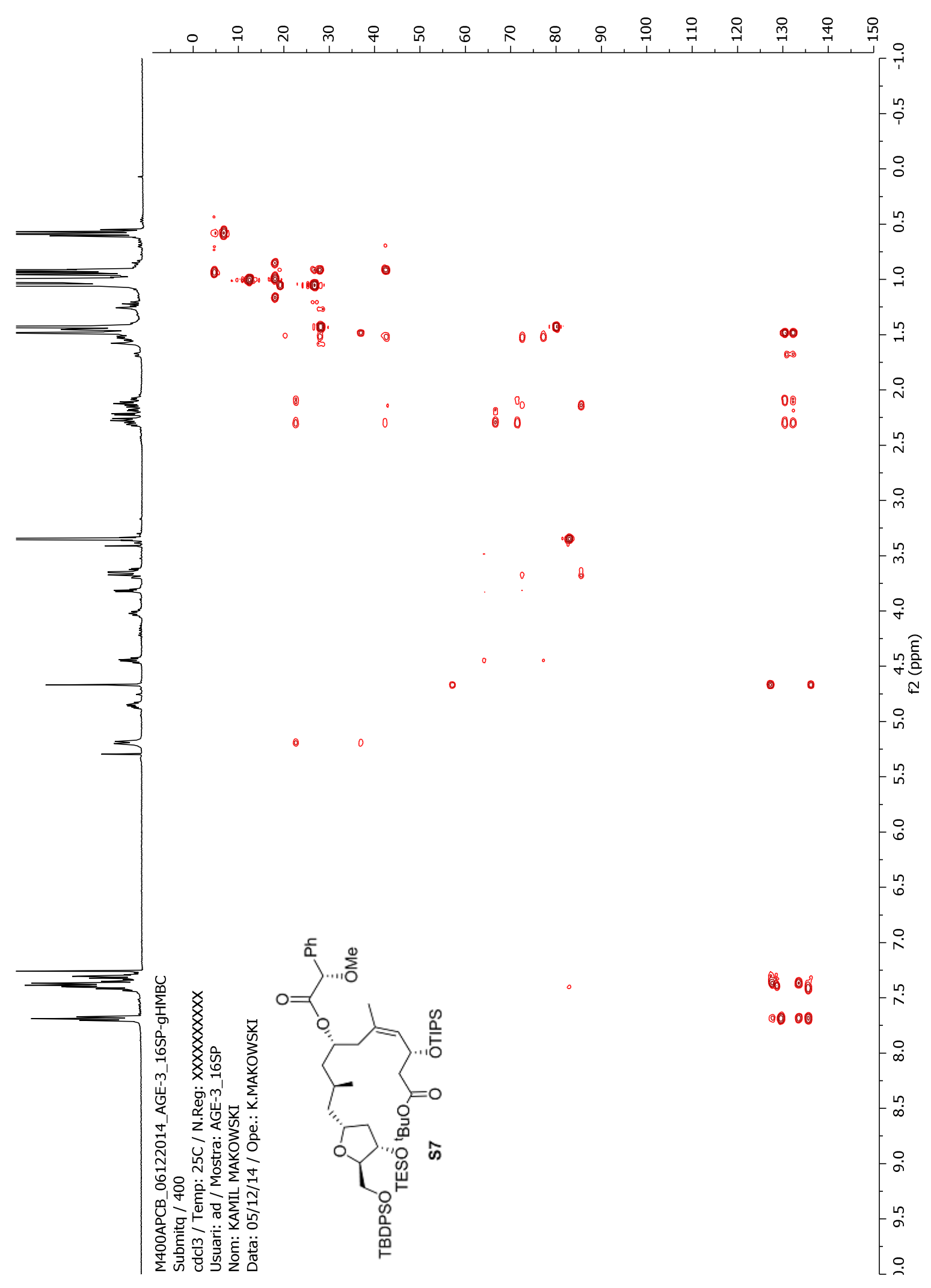




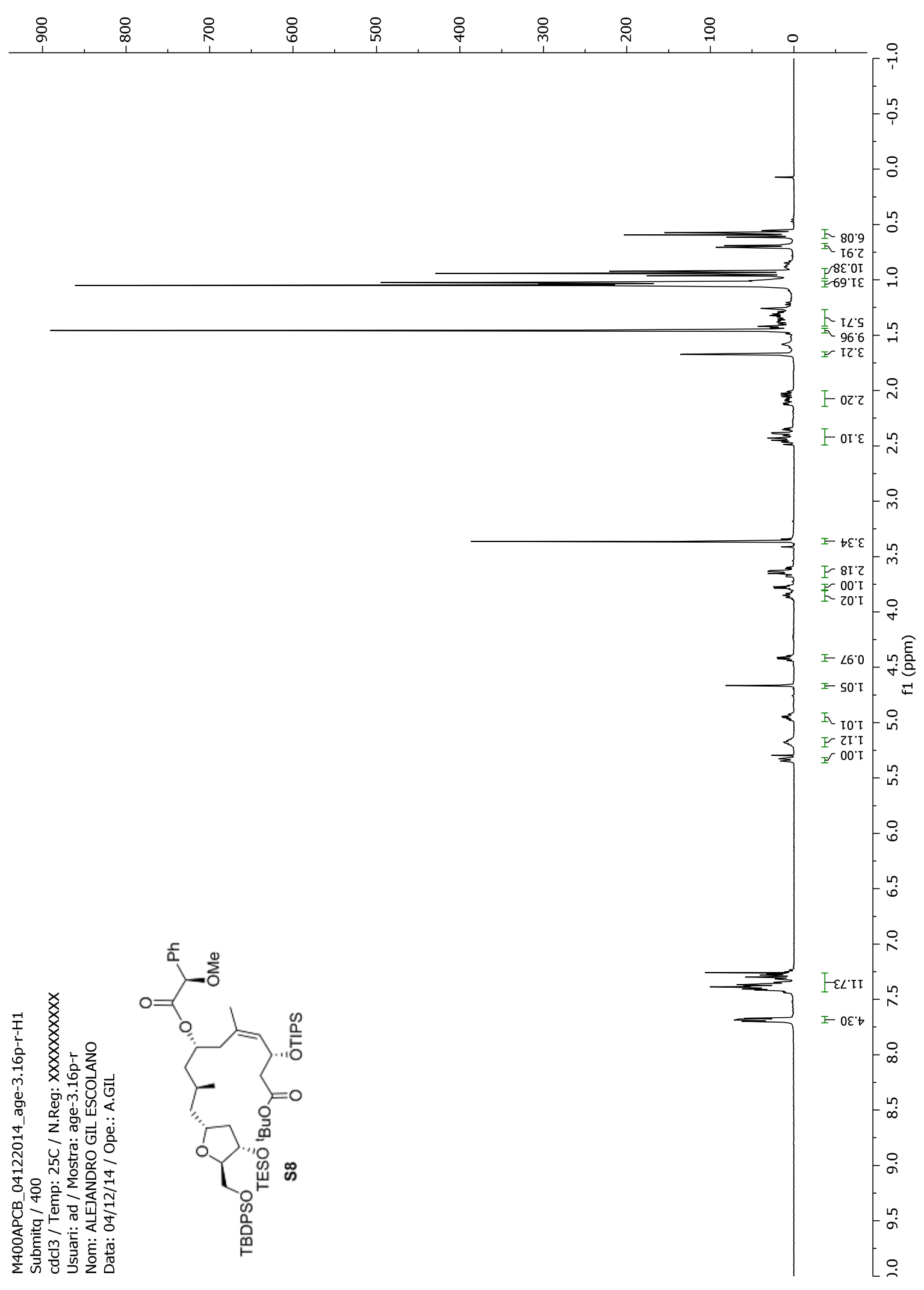


(udd) If

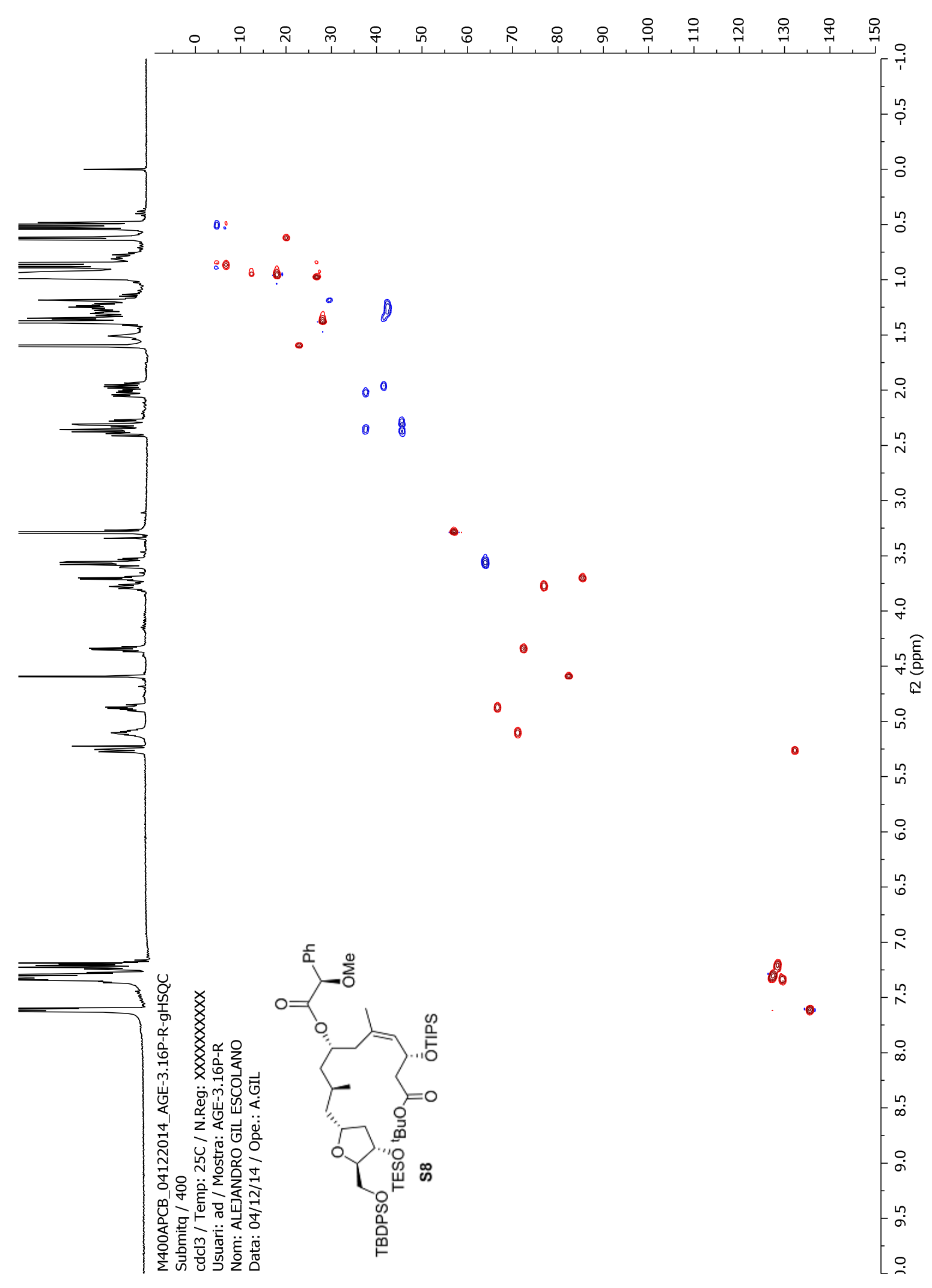


(udd) If

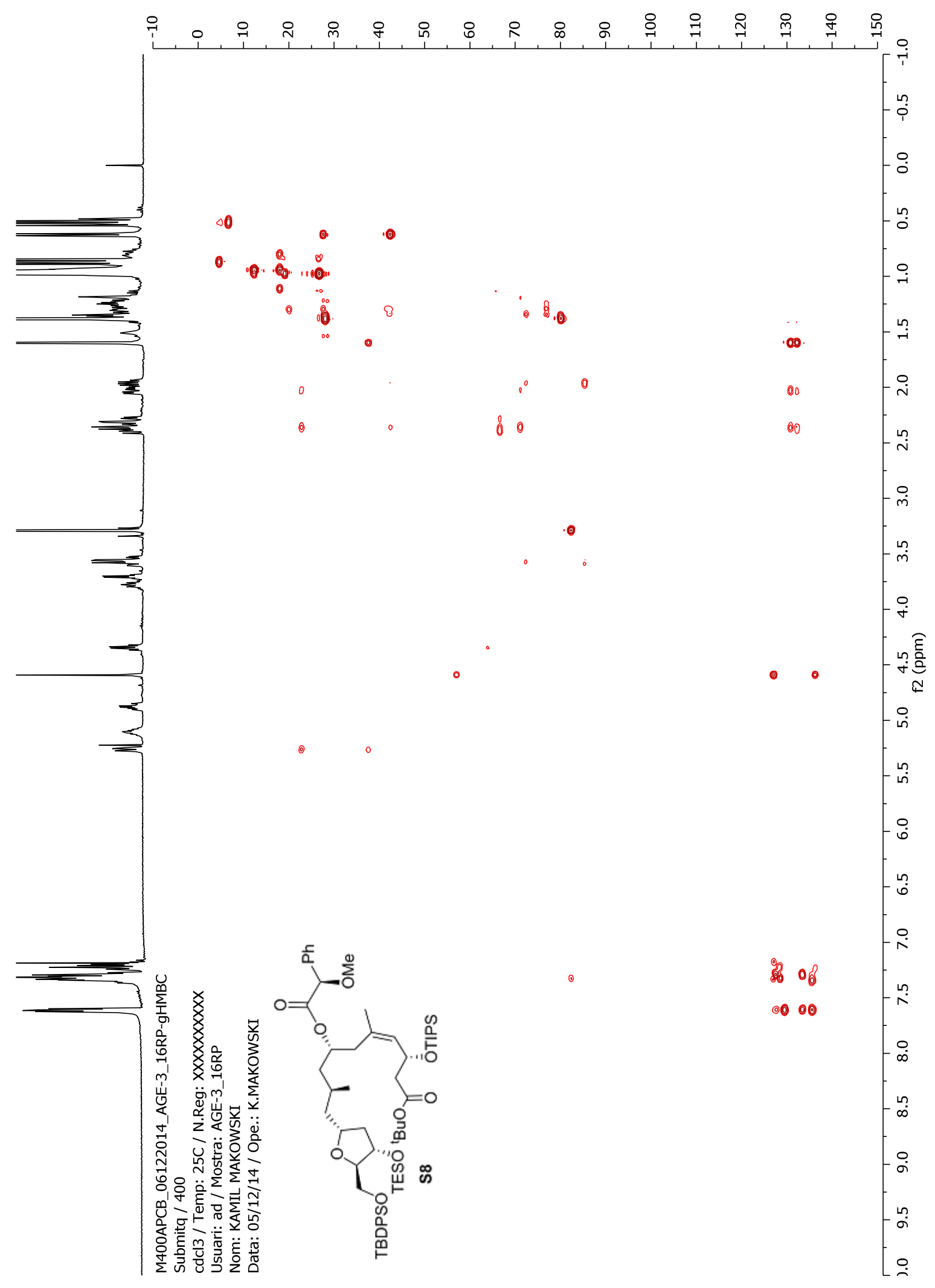

